\title{
Spectroscopic characterisation of the effects of plasma geometry on the angular distribution of X-Ray emission in HED plasmas
}

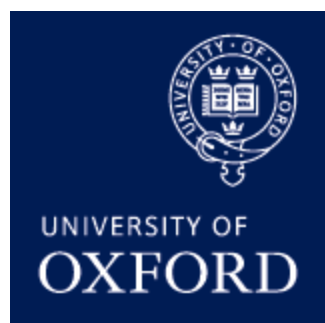

\author{
Gabriel Pérez-Callejo \\ St Anne's College \\ University of Oxford
}

A thesis submitted for the degree of

Doctor of Philosophy

Hilary Term 2020 
To my family and Mónica, for believing in me when I didn't. 


\section{Acknowledgements}

There is a lot of people that I want to thank for their help and support during these past four years. First, I want to thank my supervisor, Professor Justin S. Wark for making me the scientist I am today and making me realise that what is important is not finding an answer, but finding THE answer. Additionally, Professor Steve J. Rose, as although unofficially, his co-supervision has been a fundamental part of my development as a scientist. Their knowledge about Physics as a whole, guidance and support have been crucial for the completion of this work.

Additionally, the members of the non-LTE group from the Lawrence Livermore National Laboratory have also been a massively important part of this project, and they deserve my deepest gratitude. Special thanks go to Marilyn, for her endless patience with the guy 8 time-zones away, and for always making me feel welcome. Also to Charlie and Ed, for guiding me through every step of the process of doing experiments (including the most important one: always buy peanut donuts); and to Duane, for always having time to discuss science with me, no matter the topic.

Back in Oxford, I want to thank every member of my research group for expanding my knowledge of High Energy Density Science far beyond the extent of my own research. Specially to Orlando and Tom Preston, who dedicated a non-negligible amount of their time to help me get up to speed both with the science, and the English language 11

Oliver and Alex deserve a special mention here. They have been a massive support in the Physics department since our first pub trips after journal club in our first year. My experience during the past years has been massively shaped by their banter and peer support. Thanks also go to Rami, for always wanting to try new things and including me in those plans, regardless of how crazy they sound.

\footnotetext{
${ }^{1}$ Although I am still uncapable of pronouncing 'pun'
} 
I want to thank my housemates Antje, Nicole, Lisa and Kostas (and also Mr. Bones and Hippo...) for the board game sessions and the trips to the Oxford Blue to distract our minds from work. Complaining about work together always made it sound more fun.

Outside of Oxford, El Comando is the best group of friends this side of the Mississippi and I am incredibly happy that our yearly trips have become more frequent. I think I have managed to make them aware that I am in Oxford. Thanks must also go to Miguel, Pablo and Mateo for putting up with me during my teenage years. Although we do not see each other as much as we used to, we still manage to go for a caña when we happen to be in the same country (and city).

Thanks must also go to my parents who have not doubted me for a second. I owe them everything I am (in fact, quite literally), and I will pride myself if at some point I can say that I am at least a bit like them. Also to my sisters, who always manage to make me feel special (but I will never tell them that).

To Mónica, for being my best friend and for stopping the rain everytime she comes to visit 2 I am sure we are the current holders of the record for more and longest Skype calls, and I am proud of it. Thanks for spending the quarantine with me, and keeping me sane during those weeks. But most importantly, thanks for making me a better person.

And last, but definitely not least, I would like to thank the people who introduced me to the world of Laser and Plasma Physics, back in my days as an undergraduate. Verónica González Fernández, thanks to whom I got into Physics outreach and whose patience during my clumsiest years as an experimentalist was amazing; and specially to María Inmaculada de la Rosa, "Inma", and Klaus Grützmacher, who recently passed away. Two of the strongest people I know, and to whom I owe the world. Thank you, Inma. Thank you, Klaus, Physics is less fun without you.

\footnotetext{
${ }^{2}$ So stop wondering, John Fogerty
} 
The author gratefully acknowledges support from Lawrence Livermore National Laboratory under grant number B617350. The author wants to thank the experimental crew of the Omega laser at the University of Rochester Laboratory for Laser Energetics for expertly executing the experiments described here. The targets for those experiments were fabricated by General Atomics thanks to support from the National Nuclear Security Administration under contract DE-NA0001808.

This work was performed under the auspices of the U.S. Department of Energy by Lawrence Livermore National Laboratory under Contract DE-AC52-07NA27344 


\begin{abstract}
Understanding the plasma conditions inside hohlraums in Inertial Confinement Fusion (ICF) experiments is important to achieve ignition. However, obtaining actual measurements of those conditions is a complicated task. X-ray spectroscopy techniques offer a promising approach to these measurements, given the amount of information contained in the plasma spectra. Extracting this information from the data requires reliable spectral models that are experimentally benchmarked.

This thesis presents an analysis of the effects of the plasma geometry on its spectra, and their potential capabilities for spectroscopic diagnosis. By doing a detailed mathematical treatment of several geometries, it is shown that a cylindrical plasma is optimal to study these effects. The cylindrical geometry is therefore further studied with focus put on the emission from the $\mathrm{He}-\alpha$ complex and how the line ratios are modified by the geometry. The potential use of these ratios as a measurement of the ion density of the plasma is described.

The 'OpticalDepth' experiments at OMEGA are then detailed. In these experiments, plasma cylinders in the High Energy Density (HED) regime were generated and characterised using time-resolved X-ray imaging and $\mathrm{X}$-ray spectroscopy. The experimental results are compared with predictions from a 2-dimensional radiative transfer computational model, showing that the simulations reproduce the experimental results to a much better degree than models which do not include the plasma geometry.

The agreement found between experiment and simulations makes it possible to use cylindrical tracers in ICF experiments to obtain information about the plasma density from the geometry-modified line ratios. This is shown by analysing data from the Dot Spectroscopy campaign at the National Ignition Facility (NIF). Direct comparison of the tracers' spectra from these experiments with the predictions from the computational model developed in this work yields time-resolved measurements of the ion density of the plasma, without the need for additional diagnostics.
\end{abstract}




\section{List of Figures}

1.1 Cross section for different nuclear fusion reactions as a function of the energy of the system in the centre of mass reference frame. . . . . . . 3

1.2 Schematic drawing of the ICF process. . . . . . . . . . . . 6

1.3 Schematic drawing of the energy deposition process in indirect drive ICF. . . . . . . . . . . . . . . . . . 9

1.4 Different positions of the tracer dot along the hohlraum. . . . . . . . 12

2.1 Differential element of spectral flux and magnitudes involved in its definition. . . . . . . . . . . . . . . . 30

2.2 Schematic of the effects of the plasma geometry in the atomic populations and radiation transport. . . . . . . . . . . . . . . . . . 32

$2.3 \quad$ Schematic drawing of a cylinder with radius $R$ and thickness $H$. . . . 35

2.4 Top view of a cylinder showing the radiation path length as a function of the angle $\phi . . \ldots \ldots \ldots . \ldots \ldots$

2.5 Comparison between the exact value of the brackets in Equation 2.26, and the approximation used in this thesis. . . . . . . . . . . . . 37

$2.6 \quad$ Ratio between the face-on and the side-on flux for a cylinder of aspect ratio $a=0.2 . \ldots \ldots \ldots \ldots$

$2.7 \quad$ Schematic drawing of a hemispherical shell with radius $R$ and thickness

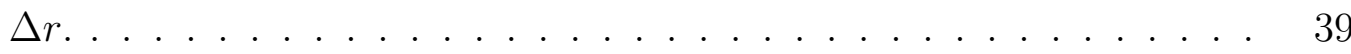


2.8 Comparison between the exact value of the square brackets in Equation 2.31 and the approximation given in Equation $\mid 2.32$. . . . . . . . . . 40

2.9 Ratio between the face-on and side-on flux for a hemispherical shell as a function of $\gamma(\nu) . \ldots \ldots \ldots$. . . . . . . . . . . . . . . . . 41

2.10 Schematic drawing of a symmetric hemi-ellipsoidal shell with radii $R_{1}$ and $R_{2}=a R_{1}$; and thickness $\Delta r$. . . . . . . . . . . . . 42

2.11 Value of $b_{\text {ell }}^{f o}(a)$ used for the exponential approximation of the face-on flux from a hemi-ellipsoidal plasma. . . . . . . . . . . . . . . . 43

2.12 Comparison between the exact value of the $G_{\text {ell }}$ integrals and the corresponding exponential approximations. . . . . . . . . . . . . . . . . . 44

2.13 Ratio between the face-on/side-on flux for optically thick to optically thin radiation in a symmetric hemi-ellipsoidal plasma as a function of its aspect ratio. . . . . . . . . . . . . . . . . . . . . . 45

2.14 Schematic diagram of a plasma torus, where $r$ is the radius of the revolving circle, and $R$ is the revolving radius. . . . . . . . . . . . . 46

2.15 Value of $b_{t o r}(a)$ used for the exponential approximation of the side-on flux from a plasma torus. . . . . . . . . . . . . . . . . . 47

2.16 Comparison between the exact value for the side-on emission of a torus, and the exponential approximation. . . . . . . . . . . . . . . . . 48

$3.1 \quad$ Configuration of the excited states of the $\mathrm{He}-\alpha$ complex. . . . . . . 53

3.2 Comparison between the emission from He-like $\mathrm{V}$ for different ion densities at a constant electron temperature of $1000 \mathrm{eV} . \quad$. . . . . . . . 54

3.3 Example of the spatial distribution of the populations of the ground and the excited states of the $w$ line transition. . . . . . . . . . . 56

$3.4 \quad$ Increase in the population of the excited state of the $y$ and $w$ transitions. 57

$3.5 G_{w}^{\text {face }} / G_{w}^{\text {side }}$ ratio as a function of the optical depth of the $y$ line for a variety of aspect ratios. . . . . . . . . . . . . . . . 62 
3.6 Values of $f_{2}(\beta, C)$ as a function of $\beta$ and $C$. . . . . . . . . . 64

$3.7 \mathrm{He}-\alpha$ spectra for the face-on and side-on detectors as predicted by Cretin, before and after the Li-like satellite emission is removed. . . . 66

3.8 Schematic of the frustum geometry and ion density values obtained from the line ratios in this case. . . . . . . . . . . . . . . . . . . . . 69

4.1 Schematic of the XRPINH design used for these experiments. . . . . . 74

$4.2 \quad$ Fibre bond faceplate that sends the phosphor signal to the CCD. . . $\quad 75$

4.3 Schematic of the elliptical spectrometer design used for these experiments. . . . . . . . . . . . . . . . . 77

4.4 Front and side view of the targets. . . . . . . . . . . . . . . 79

4.5 Different views for all crystal spectrometers and pinhole imagers for the different TOs. . . . . . . . . . . . . . . . . . . . . . 80

$4.6 \quad$ Laser drive for both TOs and percentual residual between them. . . . 81

4.7 Comparison between the power measured by DANTE for both TOs. . 82

4.8 Spectral and imaging data for both side-on and face-on views. . . . . 84

\begin{tabular}{|ll|l|l|}
\hline 4.9 & Example of face-on and side-on spectra for V, taken from Figure & 4.8
\end{tabular}

4.10 Size evolution of the targets in both the axial and radial dimensions. $\quad 87$

4.11 Example of the results from the genetic algorithm used to estimate the plasma temperature. . . . . . . . . . . . . . . . 88

4.12 Temperature and density of vanadium ions in the plasma during its expansion. . . . . . . . . . . . . . . . . . . . . . . 89

4.13 Example of face-on and side-on fits to the $\mathrm{He}-\alpha$ complex, showing the different components of the spectra. . . . . . . . . . . . . . . . . 90

4.14 Line-integrated $w / y$ flux ratio from experiment and simulations. . . . 91

4.15 Value of the opacity of the $y$ line at line centre as calculated from the experimental line ratios compared with the results from three different codes. . . . . . . . . . . . . . . . . . . 94 
4.16 Comparison between the two laser pulses used in the experiment, and the total power emitted by the plasma in both cases. . . . . . . . . . 95

4.17 Ratio of the integrated radiation flux (normalised by the pulse energy) emitted by the plasma using a square laser pulse with respect to the picket pulse, normalised to the pulse energy. . . . . . . . . . . . . 96

4.18 Expansion data from the picket pulse compared with the regular pulse. 97

$5.1 \quad$ Electron temperatures (solid line) and electron densities (dashes) as a function of time at $300 \mu \mathrm{m}$ from the original target surface. . . . . . . 102

5.2 Experimental spectrum from Chambers et al. (2002) compared with the result from the Cretin model. . . . . . . . . . . . . . . . . . . 103

$5.3 \quad$ Schematic drawing of the design of a streak camera. . . . . . . . . . . 104

5.4 Schematic of the working principle of the NXS. . . . . . . . . . 106

$5.5 \quad$ Example of spectra obtained by the IPs in the NXS. . . . . . . . . . 107

$5.6 \quad$ Schematic of the experimental set-up showing the view of the different instruments together with the shape of the laser pulse used in the experiment. . . . . . . . . . . . . . . . . 108

$5.7 \quad$ XRFC data from the showing the dots' thickness and position. . . . . 109

5.8 Streak-camera spectrum for Target 800 . . . . . . . . . . . . . . . . . 110

5.9 Temperature distributions as a function of time for both Target 800

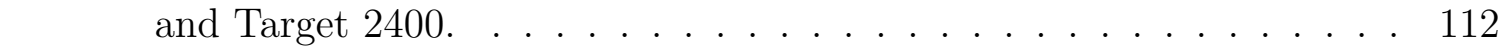

5.10 Results for the line-integrated $w / y$ flux ratio for both targets as a function of time. . . . . . . . . . . . . . . . . . . . . . 113

5.11 Map of the line-integrated $w / y$ flux ratio for Target 2400 as a function of the plasma temperature and ion density. . . . . . . . . . . . . . . 114

5.12 Ion density of both targets obtained from their $w / y$ ratio, assuming a cylindrical shape, compared with the predictions from HYDRA. . . . 116 
5.13 Comparison between the absolute flux measured in the experiment and that predicted by simulations for Target 800 and Target 2400. . . . . 117

5.14 Spectral flux from the Cretin simulations for two different ion densities differing by a factor of two. . . . . . . . . . . . . . . . . . . . . . 118

5.15 Results for the ion density of the plasma obtained using the spherical model for Target 2400 and Target 800, compared with the corresponding results from the cylindrical model and the HYDRA simulations. . 119

5.16 Axial section from the HYDRA simulations, showing the predicted dot's position and shape. . . . . . . . . . . . . . . . . . . . . . . . 121

5.17 Schematic diagram of the process used to measure the plasma density from the $w / y$ ratio. $\ldots \ldots \ldots$ 


\section{Contents}

Acknowledgements ........................ i i

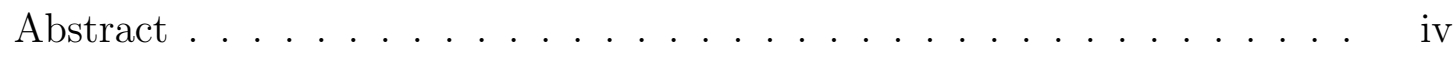

List of figures . . . . . . . . . . . . . . . . . $\mathrm{v}$

\begin{tabular}{lll}
\hline 1 & Introduction & 1
\end{tabular}

1.1 Inertial Confinement Fusion . . . . . . . . . . . . . . . . . . . . . 1

1.1 .1 Indirect drive ICF $\ldots \ldots \ldots \ldots$

1.1 .2 The Dot Spectroscopy campaign . . . . . . . . . . . . . . . 12

1.2 The radiative transfer code Cretin . . . . . . . . . . . . . . . . . . . . 14

1.3 High Power Laser facilities . . . . . . . . . . . . . . . . . . . . . . 16

1.3 .1 The OMEGA Laser Facility . . . . . . . . . . . . . . . . . . . 16

1.3 .2 The National Ignition Facility . . . . . . . . . . . . . . . . . 18

1.4 Structure of the thesis $\ldots \ldots \ldots \ldots$. . . . . . . . . . . . . . . 19

1.5 Author contributions . . . . . . . . . . . . . . . . . . . 21

$\begin{array}{lll}2 & \text { Radiative transfer and plasma geometry } & 23\end{array}$

2.1 The radiative transfer equation . . . . . . . . . . . . . . . . . 24

2.1 .1 Radiation coupling to atomic kinetics . . . . . . . . . . 25

2.1 .2 1-Dimensional solution of the radiative transfer equation . . . 27

2.1 .3 Spectral flux . . . . . . . . . . . . . . . . . . . . . 30

2.2 Enhancement of optically thick lines . . . . . . . . . . . . . . . . . 31 


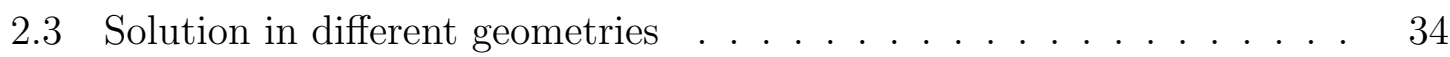

$2.3 .1 \quad$ Cylinder $\ldots \ldots \ldots \ldots \ldots$. . . . . . . . . . . . . . . . . 34

2.3 .2 Hemispherical shell $\ldots \ldots \ldots$. . . . . . . . . . . . . . 38

$2.3 .3 \quad$ Hemi-ellipsoidal shell . . . . . . . . . . . . . . . . . . . 41

2.3 .4 Torus . . . . . . . . . . . . . . . . . . . . . . . 45

2.4 Summary and conclusions $\ldots \ldots \ldots$. . . . . . . . . . . . . . . . . . . 49

3 Cylindrical plasmas in HED conditions 51

3.1 Density calculations in HED plasmas . . . . . . . . . . . . . . 52

$3.1 .1 \quad$ Interest of the $\mathrm{He}-\alpha$ complex . . . . . . . . . . . . 53

3.2 Cretin model . . . . . . . . . . . . . . . . . . . . . . . . . 55

3.3 Line ratios in cylindrical geometry . . . . . . . . . . . . . . . . . . 58

$3.4 \quad$ A fully spectroscopic method for measuring the ion density . . . . . . 60

$3.4 .1 \quad$ Aspect ratio $\ldots \ldots \ldots$. . . . . . . . . . . . . . . 61

3.4 .2 Calculation of the ion density . . . . . . . . . . . . . . 64

$3.4 .3 \quad$ A particular example . . . . . . . . . . . . . . . . . . 65

3.4 .4 Deviations from cylindrical geometry . . . . . . . . . . . . 68

3.5 Summary and conclusions . . . . . . . . . . . . . . . . . . . 70

\begin{tabular}{|lll}
4 & Experimental application: The 'OpticalDepth' campaign & 71
\end{tabular}

4.1 Diagnostic instruments . . . . . . . . . . . . . . . . . . . 72

4.1 .1 DANTE $\ldots \ldots \ldots \ldots \ldots \ldots$

4.1 .2 Framing cameras . . . . . . . . . . . . . . 74

4.1 .3 Multi Purpose Spectrometer (MSPEC) . . . . . . . . . . . 76

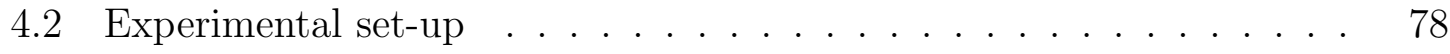

$4.2 .1 \quad$ Validation of target orientation . . . . . . . . . . . 82

4.3 Experimental results . . . . . . . . . . . . . . . . . . . . . . . . . . 83

$4.3 .1 \quad$ Target expansion $\ldots \ldots \ldots \ldots$. . . . . . . . . . 86 
4.3 .2 Plasma conditions . . . . . . . . . . . . . . . . . . . . 87

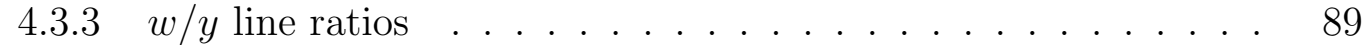

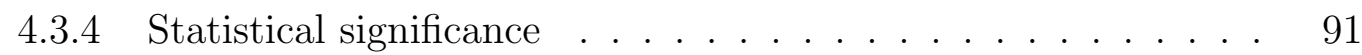

4.4 Validation of atomic models . . . . . . . . . . . . . . . . . . 92

4.5 Influence of the pulse shape $\ldots \ldots \ldots$. . . . . . . . . . . . 94

$4.5 .1 \quad$ X-ray emission $\ldots \ldots \ldots$. . . . . . . . . . . . . . . 95

4.5 .2 Expansion of the target $\ldots \ldots \ldots 6$

4.6 Conclusions $\ldots \ldots \ldots$. . . . . . . . . . . . . . . . . . . . . . . . . . . . . . 98

$\begin{array}{lll}5 & \text { Applications for ICF experiments } & 100\end{array}$

5.1 Benchmarking the spectral flux units from the Cretin model . . . . . 101

5.2 Diagnostic instruments $\ldots \ldots \ldots \ldots$

5.2 .1 Streak cameras . . . . . . . . . . . . . . . . . . 104

5.2 .2 The NIF X-ray Spectrometer ～. . . . . . . . . . . . . . . 105

5.3 Experimental set-up . . . . . . . . . . . . . . . . . . . . 107

5.4 Experimental results . . . . . . . . . . . . . . . . . . . . . . 109

5.5 Spectral analysis $\ldots \ldots \ldots \ldots$. . . . . . . . . . . . . . 111

5.6 Results for the plasma density . . . . . . . . . . . . . . . . . . 115

5.6 .1 Absolute flux . . . . . . . . . . . . . . . . . . . 117

5.6 .2 Results for spherical model . . . . . . . . . . . . . . . . . 119

5.6 .3 Discrepancies with hydrodynamic predictions . . . . . . . 120

5.7 Conclusions $\ldots \ldots \ldots \ldots \ldots$

$\begin{array}{lll}6 & \text { Conclusions and future work } & 125\end{array}$

6.1 Summary and conclusions . . . . . . . . . . . . . . . . . 125

6.2 Future work . . . . . . . . . . . . . . . . . . . . . . . . . . . . . 128

\begin{tabular}{ll}
\hline Acronyms & 132
\end{tabular} 
Contents

Bibliography 135 


\section{Chapter 1}

\section{Introduction}

This thesis describes part of the work performed by the author on X-ray spectroscopy of High Energy Density (HED) plasmas to characterise the effects of the plasma geometry on the angular distribution of their spectral emission. It contributes to the huge scientific effort aiming to measure the time evolution of the plasma conditions inside hohlraums in indirect drive Inertial Confinement Fusion (ICF) experiments. This first chapter contains a general introduction to indirect drive ICF and a description of the 'Dot Spectroscopy' experimental campaign at the National Ignition Facility (NIF), which provides a rationale for this work. Then, the radiative transfer code Cretin, used for the simulations presented in this thesis, and the laser facilities in which the experiments in this work were performed, are described. The structure of the thesis and scientific contributions of the author and others are outlined at the end.

\subsection{Inertial Confinement Fusion}

Producing controlled nuclear fusion has been one of the main goals of the scientific community since the 1950s. Controlling the extreme energy densities that are generated in burning plasmas would allow scientists to study a physical regime previously 
unavailable for laboratory experiments, where many interesting processes that have not been observed yet, are predicted to be present. In addition, the idea of a clean, renewable and efficient energy source has motivated thousands of scientists to design and develop new experimental methods and facilities that can facilitate achieving that goal.

Out of the possible fusion reactions, the one with the greatest cross section (the most probable reaction) is the fusion of Deuterium-Tritium (DT) producing an $\alpha$ particle and a neutron in the following way

$$
D+T \rightarrow \alpha(3.5 \mathrm{MeV})+n(14.1 \mathrm{MeV})
$$

This is shown in Figure 1.1, where the cross sections of several fusion rections are compared. It can be seen that for energies of the system up to $\sim 200 \mathrm{keV}$, the DT reaction is the preferrable one [1]. Given that this energy range includes the usual conditions that are achieved in fusion experiments, $\mathrm{DT}$ is the most commonly used fuel in experimental approaches to nuclear fusion.

In order for a fusion reactor to obtain energy gain, the energy resulting from fusion of the fuel, $E_{f}$ must exceed the amount of energy supplied to the plasma to heat it up to the required temperature, $E_{t}$. Neglecting energy losses, this condition can be written as

$$
n_{D} n_{T} \overline{v \sigma} E \tau>\frac{3}{2} k_{B} T\left(n_{D}+n_{T}+n_{e}\right)
$$

where $n_{D}, n_{T}$ and $n_{e}$ are the number density of deuterion ions, tritium ions and electrons respectively; $\overline{v \sigma}$ is the Maxwellian-averaged product of the ion velocity and the cross section of the fusion reaction (the reaction rate), $E$ is the energy released by a single fusion reaction, $\tau$ is the confinement time, $k_{B}$ is Boltzmann's constant and $T$ is the temperature. In the case of DT fusion, $n_{D}=n_{T}=n / 2$, where $n$ is the density of ions (both deuterion and tritium). Furthermore, given that the ions are completely 


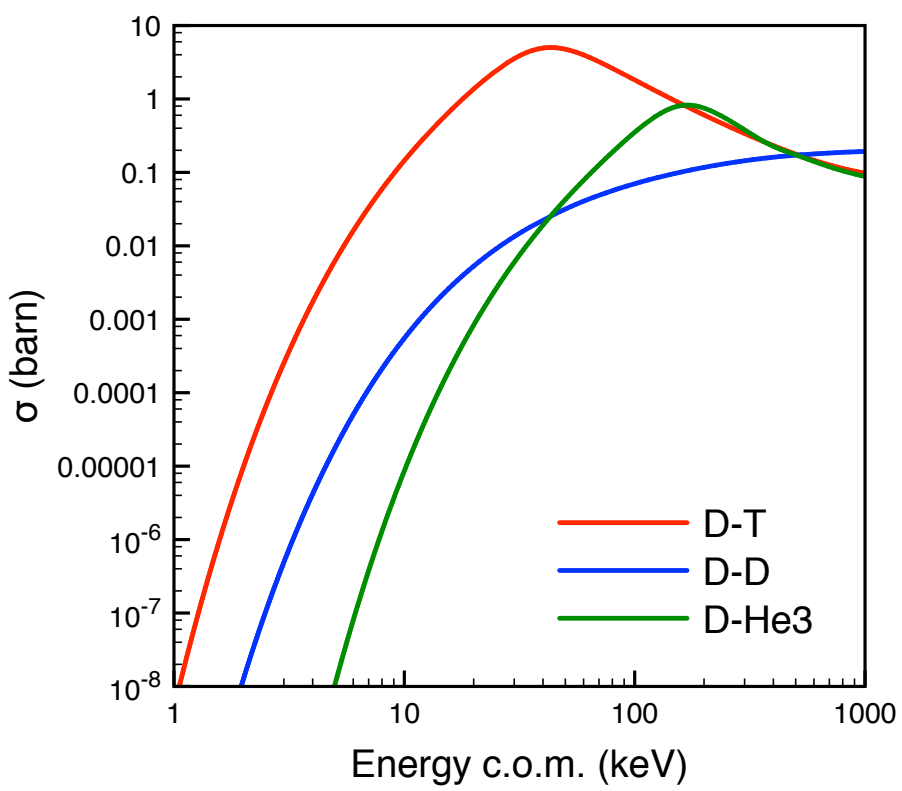

Figure 1.1: Cross section for different nuclear fusion reactions as a function of the energy of the system in the centre of mass reference frame. Note how the cross section for the D-T reaction is greater than the other cases for energies up to $200 \mathrm{keV}$. The data have been obtained from the International Atomic Energy Agency (IAEA) Nuclear Data Section - Evaluated Nuclear Data Files (ENDF) [1].

ionised at the temperatures of consideration, and hydrogen isotopes only have one electron, $n_{e}=n$. Therefore, Equation 1.2 can be rewritten (upon rearrangement of the variables) as

$$
n \tau>\frac{12 k_{B} T}{\overline{v \sigma} E}
$$

This condition is known as the Lawson criterion [2, 3]. Its physical meaning is that the denser the plasma, the less time it needs to be confined in order to obtain fusion energy, whereas a less dense plasma will need to be confined for a longer time in order to achieve fusion gain. Note that Equation 1.3 has a temperature dependence of the form $T / \overline{v \sigma}$. For a temperature of $10 \mathrm{keV}$, the condition in Equation 1.3 translates to $n \tau \gtrsim 10^{14} \mathrm{~s} \mathrm{~cm}^{-3}$.

One of the most prolific approaches to controlled nuclear fusion is the so-called Inertial Confinement Fusion (ICF), in which lasers of extremely high power are used to heat the plasma to fusion-relevant temperatures. This approach relies on the fact 
that ions in a plasma cannot move with a velocity greater than the sound speed, and therefore plasmas cannot expand infinitely fast. It is therefore possible to define a timescale $\tau$ over which an expanding plasma is confined by its own inertia (hence the name ICF . Considering a spherical plasma with radius $r$, this confinement time can be approximated as the time that the plasma takes to double its radius, that is

$$
\tau \approx \frac{r}{c_{s}}
$$

where $c_{s}$ is the sound speed. Additionally, the particle density $n$ is related to the mass density $\rho$ and the mass of a single ion $M$ as $n=\rho / M$. It is therefore possible to rewrite Equation 1.3 as

$$
\rho r \gtrsim \frac{12 k_{B} T c_{s} M}{\overline{v \sigma} E} \sim 1 \mathrm{~g} \mathrm{~cm}^{-2}
$$

which is the most common way of expressing the Lawson criterion in ICF. The product $\rho r$ is a particularly interesting figure of merit in $\mathrm{ICF}$, as the mean-free-path (mfp) of the products generated in the reaction is usually written in terms of this parameter. In particular, the $\alpha$ particles resulting from DT fusion have a $\mathrm{mfp}$ of $\sim 0.3 \mathrm{~g} \mathrm{~cm}^{-2}$ at $10 \mathrm{keV}$.

With this condition, it is now possible to calculate the amount of energy necessary to generate an inertially-confined DT plasma at $10 \mathrm{keV}$ such that the Lawson criterion is fulfilled. Neglecting energy losses again, the thermal energy that needs to be supplied to the plasma, as shown in Equation 1.2, is

$$
E_{\text {laser }}=3 n k_{B} T \frac{4 \pi r^{3}}{3}
$$


which, combined with the condition in Equation 1.5 becomes

$$
E_{\text {laser }}=\frac{4 \pi k_{B} T}{\rho^{2} M}(\rho r)^{3} \gtrsim \frac{5}{\rho^{2}} \mathrm{GJ} \mathrm{g}^{2} \mathrm{~cm}^{-6}
$$

It can be seen that the energy required decreases with the square of the mass density of the fuel. If uncompressed solid DT $\left(\rho \sim 0.25 \mathrm{~g} \mathrm{~cm}^{-3}\right)$ were used as fuel, several gigajoules of laser energy would be required to fulfill the Lawson criterion. This exceeds by several orders of magnitude the power of the most powerful laser in the world at the time of writing this thesis $(\sim 2 \mathrm{MJ})$. It is for this reason that the DT fuel needs to be compressed to $\sim 100$ times its solid density in order for fusion to be technologically feasible.

An estimate of the pressures required to compress the plasma to such high densities, can be obtained from quantum statistics. When the density of the fuel is increased to several times the solid density of the material, the free electrons within the plasma will follow a Fermi-Dirac distribution. Therefore, the minimum pressure that needs to be applied to the plasma to reach such high densities, is given by the Fermi pressure, as

$$
P_{F}=\frac{\hbar^{2}\left(3 \pi^{2}\right)^{2 / 3}}{5 m_{e}} n_{e}^{5 / 3}=\frac{\hbar^{2}\left(3 \pi^{2}\right)^{2 / 3}}{5 m_{e}}\left(\frac{\rho}{M}\right)^{5 / 3}
$$

For densities of the order of 100 times the solid density of DT, this results in several thousands of MBar. However, in practical applications, laser pressures are limited to $\sim 100$ Mbar [4], far below the values that are necessary to achieve the required compression.

ICF targets usually present a layered design in which a sphere of low-density DT gas $\left(\rho<1 \mathrm{mg} \mathrm{cm}^{-3}\right)$ is enclosed by a thin layer of solid or liquid DT, which constitutes the main fuel, and contains $\sim 95 \%$ of the DT mass. This shell is covered with an additional layer, usually made from parylene $(\mathrm{CH})$ or High Density Carbon 


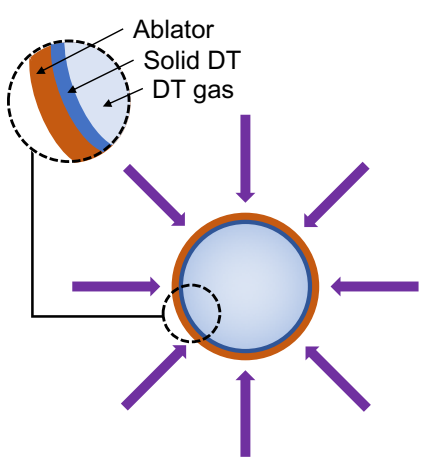

1. Energy deposition

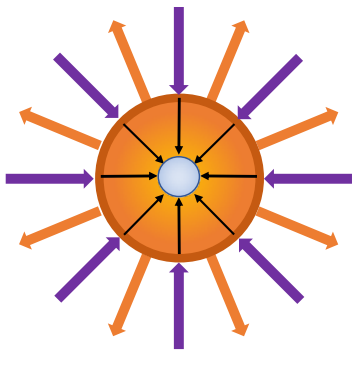

2. Initial compression

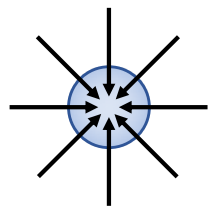

3. Fusion at the hot spot

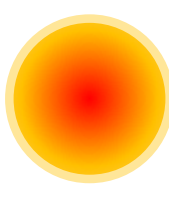

4. Ignition

Figure 1.2: Schematic drawing of the $\mathrm{ICF}$ process. First, the laser energy is deposited on the ablation shell, initiating the compression of the fuel capsule. Secondly, as the ablation shell expands the reaction forces compress the fuel. In the third step, the compression stops (stagnation), and fusion reactions start being produced at the hot spot, bootstraping it temperature to several tens of keV. Finally, a burning wave is propagated to the cryogenic layer, driving more fusion reactions. Ignition is achieved and the reaction becomes self sufficient.

(HDC), called the ablator. Due to the presence of the cold layer of solid or liquid DT, these targets are usually called cryogenic targets. The design is shown in the enlarged region of step 1 in Figure 1.2 .

In the so called direct drive approach to $\mathrm{ICF}$, the laser beams impinge directly upon the fuel capsule, depositing their energy on the ablator [5, 6, 7], thus creating a plasma on its outer surface. This experiments are usually carried out in vacuum, and therefore this ablator plasma expands outwards from the fuel. Similarly to a rocket mechanism, as mass is being ablated, a reaction force acts inwards (the ablation pressure), compressing the fuel to several times its solid density. With the usual experimental designs, at the point of maximum compression, the nominal density of the DT gas is typically $\sim 75-100 \mathrm{~g} \mathrm{~cm}^{-3}$, and that of the solid DT is $\sim 1000 \mathrm{~g} \mathrm{~cm}^{-3}$ [4, 8].

As the capsule is compressed, the pressure across the DT fuel is kept constant [9], which implies that the product $\rho T$ is also constant. Due to the steep change in density from the $\mathrm{DT}$ ice shell to the $\mathrm{DT}$ gas, this condition means that the temperature in 
the central region of the capsule is much higher than that of the DT shell around it. This high-temperature, low-density region is called the hot spot, and it is where fusion reactions start being produced. The experiments are commonly designed so that the hot spot has an areal density of $\geq 0.3 \mathrm{~g} \mathrm{~cm}^{-2}$, so that the alpha particles produced in the fusion reactions deposit their energy within the hot spot, and bootstrap its temperature to several tens of $\mathrm{keV}$ [4, 8]. The $\alpha$ particle deposition in the hot spot generates a burn wave which propagates to the solid DT shell, where most of the fuel mass is contained, triggering more fusion reactions. The point at which the process becomes self-sustainable by the effect of this $\alpha$-heating is called ignition. This process is schematically illustrated in Figure 1.2 .

The combination of ablation and spherical convergence in these targets can increase the laser pressure by several orders of magnitude [4, 5, 10, 11]. To a simple approximation, the velocity of the imploding shell of solid DT (which contains most of the fuel) is related to the ablation pressure through Newton's second law as

$$
P_{a} S=\rho S \Delta r \frac{\partial v}{\partial t}
$$

where $S$ is the surface of the shell and $\Delta r$ is its thickness. This equation yields the following solution for the velocity of the implosion as the radius of the shell decreases

$$
v(R)=\sqrt{\frac{2\left(R_{0}-R\right) P_{a}}{\rho \Delta r},}
$$

where $R_{0}$ is the initial radius of the shell. If the shell and the laser drive are completely spherically symmetric, at some point the implosion stops (this is referred to as stagnation $)$, and $\left(R_{0}-R\right) \sim R_{0}$. At this point, the kinetic energy of the imploding shell is converted to internal energy of the fuel. Given that pressure is energy per 
unit volume,

$$
P_{\text {stag }}=\frac{E_{\text {kinetic }}}{V}=\frac{m}{2 V} v_{\text {stag }}^{2}=\frac{\left(R_{0}-R\right) P_{a}}{\Delta r} \sim P_{a} \frac{R_{0}}{\Delta r}
$$

Therefore, the ablation pressure can be amplified several times by using capsule designs with a high value of the so-called In Flight Aspect Ratio (IFAR), defined as $R_{0} / \Delta r$

However, the IFAR cannot be arbitrarily increased. During the implosion, a light fluid (the ablator) is accelerated towars a denser fluid (the $\mathrm{DT}$ ice) at the ablation surface. This situation is hydrodynamically unstable and small perturbations will grow exponentially in time due to what is called the Rayleigh-Taylor (RT) instability. This instability alters the symmetry of the implosion, preventing the compression. Using a simple model, the growth rate $\gamma$ of the RT instability for direct drive ICF can be approximated as

$$
\gamma^{2}=k a(1-k \Delta r)
$$

where $k$ is the mode wave number and $a$ is the acceleration of the capsule [5, 10]. It can be seen that all the wave modes with $k>1 / \Delta r$ have a negative growth rate and therefore do not contribute to the instability. In fact, the growth rate is maximum for $k=1 / 2 \Delta r$. To get an estimate of how much the instability can grow during the implosion, the acceleration can be substituted for its value at stagnation, which can be obtained by solving Equation 1.9 and corresponds to $a=\sqrt{2 R_{0} / t^{2}}$. Therefore, the maximum number of e-foldings that the instability can grow before stagnation is

$$
\gamma t=\sqrt{\frac{R_{0}}{2 \Delta r t^{2}}} t=\sqrt{\frac{R_{0}}{2 \Delta r}} .
$$

From this equation it follows that, although increasing the IFAR is desirable to increase the pressure on the fuel (as shown in Equation 1.11), there are physical limi- 


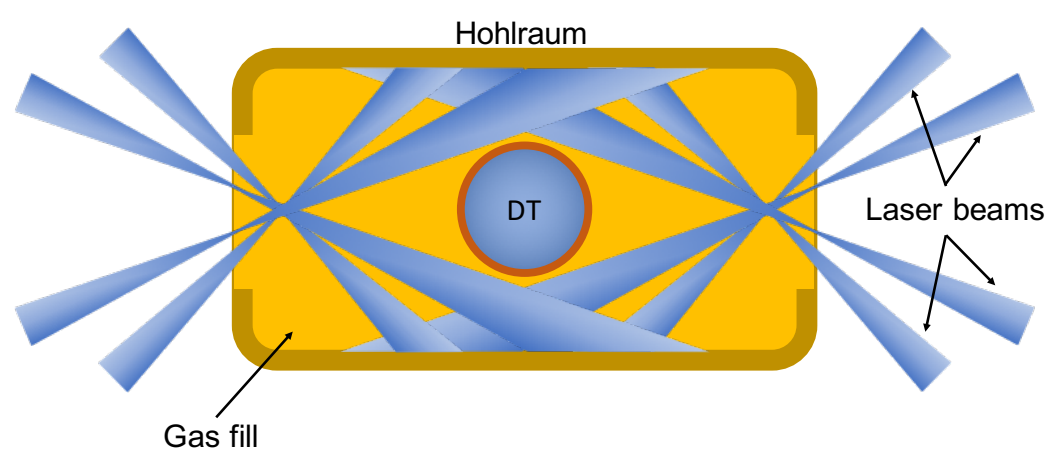

Figure 1.3: Schematic drawing of the energy deposition process in indirect drive ICF.

tations to the values it can take to avoid break-up of the shell. In usual [CF experiments, the IFAR is limited to values of $25-40$ to achieve optimum compression while minimising the growth of the RT instability.

In practice it is impossible to avoid the generation of the RT instability, as it is physically impossible to drive the compression with an infinite number of perfectly smooth laser beams, which would be necessary to generate a perfectly uniform drive on the target. In particular, in direct drive $\mathrm{ICF}$, the laser beams require a high degree of smoothing, as any speckle pattern in their imprint on the target can trigger the RT instability.

\subsubsection{Indirect drive ICF}

Due to the high degree of beam smoothing required for direct drive $\overline{I C F}$ and the technical difficulties it implies, the indirect drive approach to ICF was developed. As its name indicates, the main difference with respect to direct drive lies in the way in which the laser energy drives the fuel compression.

In indirect drive ICF [10, 12, 13, 14], a cryogenic DT capsule of $\sim 1 \mathrm{~mm}$ in radius is mounted inside what is called a hohlraum (German for 'hollow room'). These are small cylinders usually made from gold, about $1 \mathrm{~cm}$ long and $5 \mathrm{~mm}$ in diameter. Contrary to the direct drive approach, the lasers are not shone directly onto the 
capsule, but they impinge upon the inner walls of the hohlraum, transferring their energy, as illustrated in Figure 1.3. This generates a gold plasma inside the hohlraum which surrounds the DT capsule and emits a Planckian radiation field that drives the fuel compression. At the temperatures that the gold plasma reaches $(\sim 300 \mathrm{eV})$, this Planckian field consists mostly of X-ray radiation. Although the energy coupling between the laser and the fuel is not as efficient as in the direct drive approach, due to the conversion of the laser energy to X-rays; the radiation field from the gold plasma surrounding the fuel produces a more spherically symmetric compression.

If the lasers impinged upon the hohlraum in vacuum, the gold plasma from the walls would quickly expand and fill the hohlraum, getting in the way of the laser beams and absorbing part of their energy. This would introduce several problems, as the points on which the laser energy is deposited would change with time (as the gold plasma filled the hohlraum), and some regions of the hohlraum would become inaccessible to the laser beams, modifying the X-ray distribution. Additionally, the efficiency of the energy conversion from the laser into the X-ray field would be further reduced. Although this effect could be mitigated by using larger hohlraums, the total laser energy available with the current facilities limits the ratio between the radius of the hohlraum and that of the capsule, or case-to-capsule ratio (CCR), to values of $3-4$ [10, 15, 16], which is not enough to avoid the gold plasma getting in the way of the laser beams.

To slow down the expansion of the gold plasma, hohlraums are usually filled with a gas [17, 18, 19], typically helium at $\sim 1 \mathrm{mg} / \mathrm{cm}^{3}$. However, the gas fill introduces additional complications, as depending on the density of the gas, several Laser Plasma Interaction (LPI) processes will be produced inside the hohlraum. In particular, Cross Beam Energy Transfer (CBET) caused by Stimulated Brillouin Scattering (SBS) can greatly modify the symmetry of the implosion, and hot-electron generation by Stimulated Raman Scattering (SRS) can pre-heat the DT fuel before peak compression. 
Furthermore, backscatter of laser radiation from SRS and SBS reduces the amount of laser energy that is deposited in the hohlraum, and thus the efficiency of the process is greatly reduced [20, 21, 22].

The interactions between the laser radiation, the gold plasma from the hohlraum walls, the gas fill, the X-ray field, and the plasma from the DT capsule are extremely complicated. Modelling the evolution of these processes and how they affect the conditions inside the hohlraum during an $\mathrm{ICF}$ implosion, requires a deep understanding of LPI processes and expensive multidimensional radiative hydrodynamic simulations. On top of that, the results from those simulations are model dependent, and experimental data that can help benchmark them are scarce. This thesis focuses on one particular technique that uses spectroscopy to obtain measurements of the evolution of the plasma temperature inside the hohlraum.

Given the great amount of information that can be extracted from the X-ray spectra of plasmas, X-ray spectroscopy is a fruitful approach to characterising the plasma conditions in $\mathrm{ICF}$. Of particular interest for this thesis are the K-shell spectral lines. These lines are produced when an electron decays from any energetic state with principal quantum number $n>1$, to the K-shell (group of states with $n=1$ ). For the elements considered in this work, the energy of the photons produced in these transitions lies in the X-ray regime. Given that the energy of the lines depends on the number of electrons screening the Coulomb potential from the nucleus, that is the charge state of the ions, in a general plasma, where several ionisation stages are present at the same time, the K-shell spectrum usually presents several lines with different energies, corresponding to the same electronic transition in different charge states. Therefore, studying the relative intensities of these lines can provide information about the relative abundance of these charge states in the plasma and their atomic population. Since the population balance between different atomic states is strongly linked to the temperature and density of the plasma, the K-shell spectra 


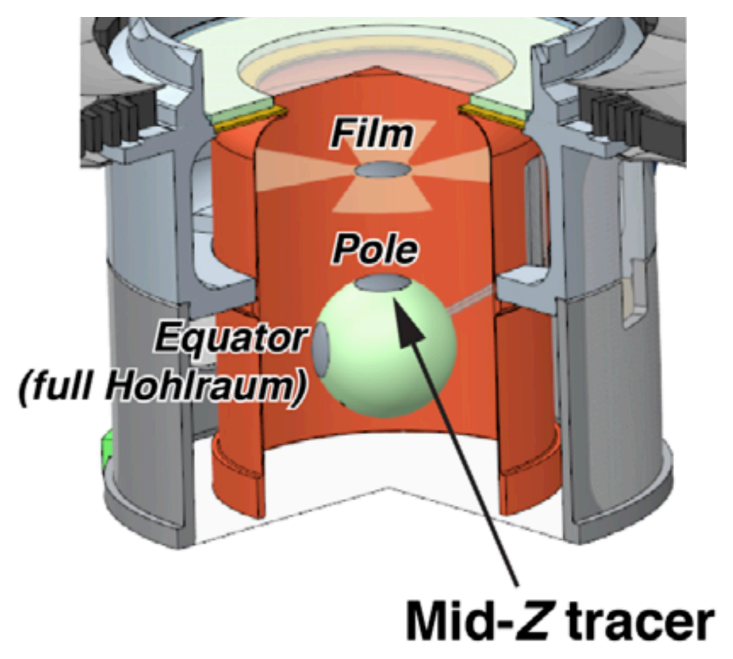

Figure 1.4: Different positions of the tracer dot along the hohlraum. The experiments with the dot at the pole of the capsule or on film were performed in truncated hohlraums whereas the equator dots were fielded in full hohlraums. $?^{1}$

can be used to map these conditions.

\subsubsection{The Dot Spectroscopy campaign}

One of the approaches to using K-shell spectroscopy in ICF, is the Dot Spectroscopy campaign, which comprises a series of experiments that were carried out at the $\mathrm{Na}$ tional Ignition Facility [23] (NIF) with the aim of using K-shell spectroscopy to diagnose the evolution of the electron temperature inside a hohlraum during an ICF implosion.

The idea of the dot spectroscopy has been around for a few decades [24, 25]. In fact, it was already studied for diagnosing the conditions inside hohlraums in indirect drive ICF, in the late 1990s at the laser NOVA (precursor of NIF] [26, 27], but only recently these experiments have been fielded at the NIF [28, 29]. The key feature of this campaign is the addition of a mid-Z tracer dot in the hohlraum. The dot can be placed in different regions of the hohlraum, such as the pole of the DT capsule, above it, or in the equator, as shown in Figure 1.4. The experiments with the dot on

\footnotetext{
${ }^{1}$ Reprinted figure with permission from $[29$. Copyright 2018 by the American Physical Society.
} 
the capsule or over it were performed in truncated hohlraums such as the one shown in the figure, whereas the dot-in-equator experiments used full hohlraums.

The dots' plasma generated in these experiments, absorbs an immense amount of energy within the span of a few nanoseconds, heating up to temperatures in excess on $1 \mathrm{keV}$ while staying at high particle densities, a regime commonly known as High Energy Density (HED) [30]. This term will be used throughout this thesis to refer to the conditions of the plasmas of study.

At the temperatures and densities characteristic of $\mathrm{HED}$, the mid-Z dot tracer gets highly ionised and starts emitting K-shell X-rays. As the atomic number of the dot (Z $\sim 20-30)$ is very different from that of the ICF fuel $(Z=1)$, or the hohlraum (usually $\mathrm{Z}=79$ ), its K-shell emission can be resolved without the lines overlapping with any contribution from other elements in the experiment. The experimental set-up of the Dot Spectroscopy experiments is discussed in more detail in Chapter 5 .

Given that the dot's spectrum changes over time as a result of the changes in its temperature, by time-resolving the K-shell line emission of the dot, it is possible to study the evolution of the temperature inside the hohlraum during the implosion [28]. Furthermore, if the position of the dot is also measured, one can link the obtained temperature evolution to a particular region of the hohlraum. By studying different positions of the dot, as shown in Figure 1.4, it is possible to experimentally map the temperature evolution in the hohlraum during an ICF implosion [29].

However, studying the K-shell emission of the dots is not trivial, as they are not spherically symmetric. The cylindrical geometry of the dots modifies the relative intensity of certain spectral components, through a mechanism widely known in astrophysics (discussed further in Chapter 2). As a consequence, depending on the direction of observation, the temperature values that are inferred from the spectra will be different. This is far from ideal, as the plasma conditions are not directiondependent, and therefore the measurements obtained without accounting for the tar- 
gets' geometry might be misleading. This can lead to using wrong models in the design of future experiments.

This thesis is dedicated to characterising how the cylindrical geometry of plasmas in $\mathrm{ICF}$ relevant conditions, such as these tracer dots, modifies their K-shell line emission, and what information about the plasma conditions can be extracted from these geometric modifications.

\subsection{The radiative transfer code Cretin}

Although an analytical approach to studying the X-ray emission from HED plasmas (such as the aforementioned dots) is not practical in most cases, there are computational tools available which can be used to study complex spectra in a simpler manner. The code Cretin [31] studies the way in which a radiation field moving within a plasma interacts with its surrounding medium, and eventually produces a spectrum that can be observed. Its name (one of the users' first questions) comes from contracting the word accretion, as it was originally an astrophysics code used to study and model accretion disks. This code is used throughout this thesis to produce spectral simulations that can be compared with the experimental and theoretical results that are presented.

According to its user's manual "Cretin is a 1-, 2- and 3-dimensional non-LTE atomic kinetics / radiation transfer code". The code can be used to study the time evolution of a plasma system, as it self consistently solves the population distribution of the atomic states in the plasma with the interaction with the radiation field within it. The atomic data used for a simulation can either be externally supplied or calculated by the code using a screened-hydrogenic formalism [32].

With respect to the atomic kinetics calculations, Cretin works with two different radiation fields which are treated differently during the solution process. The user's 
manual refers to these fields as continuum and line radiation. The line radiation is used to treat those photoexcitations which are designated as requiring special treatment, due to importance in the atomic kinetics of that element, or the particular interest they have for the user. The line radiation field for each transition should resolve the corresponding line profile, from line centre to a photon energy far enough in the wings of the line so that the broadening is completely resolved. As each element in a plasma can include an extremely large number of photoexcitations, the code does not deal with individual lines unless they are directly specified. The continuum radiation is used for an approximate treatment of all other photoexcitations, as well as for treating all other radiative transitions. This includes bremsstrahlung and radiative recombination, both of which must be integrated over a wide range of photon energies. The continuum radiation must cover all photon energies between zero and an energy above which radiative emission may safely be neglected, usually $\sim 10-20$ times the energy equivalent to the maximum electron temperature. The continuum and line radiation fields are both used when calculating the atomic populations, and are obtained from radiation transport calculations using the atomic populations. Self-consistency of the populations and radiation fields is obtained by iteration, with special iterative methods used for the line radiation fields [33].

A third radiation field, termed the 'spectral radiation', is used only after obtaining a self-consistent solution for the populations, continuum radiation and line radiation fields. The user can define this radiation to include photon energies over any sub-range of the continuum radiation and with any spectral resolution. Radiation transport with absorption and emission fixed by the populations is then used to calculate highresolution spectra without requiring the use of a similar resolution for the continuum radiation during the iterative process.

The simulations included in this work use the 2-dimensional mode of Cretin. To obtain accurate spectra, fine-structure atomic data for the H-like through Be-like 
charge states were generated up to principal quantum number $n=10$ with the Flexible Atomic Code [34] (FAC), For the H-like through Li-like charge states, the fine structure was retained up to $n=4$ and averaged to superconfigurations for $n>4$. For the Be-like charge state, all the data was averaged to superconfigurations. The FAC data was augmented by screened-hydrogenic data generated by Cretin for the other charge states, with a matching atomic structure for the Be-like charge state. These data sets were provided by H. A. Scott. In addition, the He- $\alpha$ spectral lines (see Section 3.1.1 were explicitly included in the line radiation field in the simulations. A detailed description of this computational model is given in Section 3.2 .

\subsection{High Power Laser facilities}

As mentioned in Section 1.1, in ICF experiments large amounts of laser energy are deposited onto the DT fuel either directly or indirectly (via X-ray emission from a hohlraum). To generate the high powers required to deliver that energy within a few nanoseconds, specialised facilities are needed. The next two sections describe laser facilities on which both direct and indirect drive ICF experiments are performed (among many other laser-plasma experiments). These facilities are of particular interest for this thesis, as the experiments described in Chapters 4 and 5 were performed there.

\subsubsection{The OMEGA Laser Facility}

The OMEGA laser facility is located at the Laboratory for Laser Energetics (LLE) in the city of Rochester, New York (USA). It currently comprises two independent laser systems, namely OMEGA 60 [35, 36] and OMEGA EP (extended performance) [37, 38]. It is a neodymium glass-based laser whose fundamental wavelength is $1053 \mathrm{~nm}$. This light is then frequency tripled within the laser system to finally deliver $3 \omega$ radiation $(\lambda=351 \mathrm{~nm})$ onto the targets. 
Originally, OMEGA had 24 laser beams capable of delivering $2 \mathrm{~kJ}$ of energy, until the system was upgraded between 1990 and 1995. During this upgrade, the OMEGA 60 system was built. It consists of 60 laser beams that can deliver up to $30 \mathrm{~kJ}$ of energy for a 1 ns square pulse, with a repetition rate of 1 shot/hour. The laser system is designed to deliver arbitrary pulse shapes between $0.1-4 \mathrm{~ns}$ long, provided that the power does not exceed $45 \mathrm{TW}$ [39]. The OMEGA EP extension was opened in 2008. It added 4 beams with the particularity that while all of them have a long pulse capability two of them can also deliver short pulses. When the beams are working in long-pulse mode, they can deliver 0.1 - $10 \mathrm{~ns}$ pulses at energies up to $6.5 \mathrm{~kJ}$ per beam. Additionally, the short-pulse beams can deliver pulses between $1-100 \mathrm{ps}$ with a maximum of $2.6 \mathrm{~kJ}$ per beam. Furthermore, the two beams that have both short- and long-pulse capabilities can deliver their energy to both the OMEGA 60 and OMEGA EP chamber, whereas the pulses with only long-pulse capabilities can only deliver to the OMEGA EP chamber.

For the remainder of this thesis, focus will be put on the OMEGA 60 laser, hereafter referred to as OMEGA, where the 'OpticalDepth' experiments described in Chapter 4 were performed. The target chamber is $3.3 \mathrm{~m}$ in diameter, and its design was intended for direct drive ICF implosions [40]. For this reason, it has a football-geometry with the 60 laser beams distributed in the centre of the flat pentagons and hexagons around the target chamber to produce a drive on the target as spherically symmetric as possible. However, the wide range of pulse shapes and the laser power achievable with OMEGA have allowed experimentalists to use this facility for many different experiments such as cylindrical imposions [41], indirect drive ICF [42], studying hydrodynamic instabilities [43, 44] or even the generation of magnetic fields in the universe [45], to name a few. 


\subsubsection{The National Ignition Facility}

The NIF is an ICF-dedicated research facility located at the Lawrence Livermore National Laboratory (LLNL) in Livermore, California (USA). As is the case with OMEGA (and most high-power optical lasers) NIF is a neodymium glass-based laser whose frequency is then tripled to generate the $3 \omega$ light that is finally shone on the target.

Construction of the NIF was finished in 2009, and immediately after, in 2010, the National Ignition Campaign (NIC) [46] started. This was a series of upgrades to the NIF and dedicated experiments with the aim of reaching ignition. During this period the energy of the pulse was raised from $\sim 1 \mathrm{MJ}$ to a maximum value of $1.8 \mathrm{MJ}$, reached on the 5th of July, 2012. However, this progress was not as smooth as initially foreseen, due to several unexpected issues arising as the power was being increased to values never reached before; such as the potential damage to the optical components in the laser or the difficulty of manufacturing fuel capsules with the required degree of smoothness and purity. The $\mathrm{NIC}$ was officially ended in September of 2012 without having reached ignition.

The laser system at NIF consists of 192 beams that can now deliver up to $2.15 \mathrm{MJ}$ [47] and $500 \mathrm{TW}$ of $3 \omega$ light with pulses of up to $20 \mathrm{~ns}$ [48]. Contrary to OMEGA, NIF is designed to operate mainly in the indirect drive approach and so the distribution of the beams around the $10 \mathrm{~m}$ diameter target chamber is not spherically symmetric. In this case, there are four coaxial cones of beams, two mounted on the top and two on the bottom of the chamber, symmetric with respect to the chamber centre. Out of each pair of cones, one of them has a wider opening angle than the other. The vertices of those cones lie on the axis of the hohlraum, so that the beams from each cone hit the inside of the gold walls with cylindrical symmetry, as illustrated in Figure 1.3. This generates four ring regions (one per cone) of gold plasma, symmetric with respect to the centre of the hohlraum, whose X-ray emission improves the sphericity 
of the implosion.

Although ignition has not yet been achieved at NIF (at the time of writing this thesis), there is an immense experimental effort aimed towards this goal, and several experiments are regularly performed there with the aim of studying ICF-related parameters such as the symmetry of the implosion [49, 50, 51] or the hohlraum geometry, with new designs that can improve the results [52, 53]. Among the ICF-related experiments performed at $\mathrm{NIF}$ is the 'Dot Spectroscopy' campaign described in Section 1.1.2, and further discussed in Chapter 5 .

\subsection{Structure of the thesis}

This thesis focuses on the author's effort towards characterising the effect of the plasma geometry on its spectra, by studying plasma cylinders in HED conditions. The main part of this thesis is the design, performance and analysis of the experiments of the OpticalDepth campaign at the OMEGA laser facility, and the use of spectroscopy and X-ray imaging to benchmark these geometric effects. The work is structured in six chapters, organised as follows.

Chapter 1 contains a general introduction to the topic of $\mathrm{ICF}$. The laser facilities in which the experiments discussed in this thesis were performed are described here, together with the Dot Spectroscopy campaign at NIF and the rationale for this work. The last parts of this chapter are the structural description of the thesis and an outline of the author's contribution to the different parts of this work.

Chapter 2 is dedicated to the study of the radiative transfer equation. The main physical quantities involved in this work such as the opacity and the optical depth are then defined, and a historic discussion of the effects of the plasma geometry on its X-ray spectrum is included. Finally, the radiative transfer equation is solved exactly for several different geometries, with the aim of showing which geometry is 
optimum to study those effects. The results from this chapter were published in Journal of Quantitative Spectroscopy and Radiative Transfer [54], and High Energy Density Physics [55] in 2019. The text in the chapter is partly reproduced from those publications.

Chapter 3 describes the Cretin model used for the simulations in this thesis. A mathematical method to calculate the ion density of cylindrical HED plasmas is then presented, and an example of the application of this method is shown using the Cretin model. The density calculation method in this chapter was published in High Energy Density Physics in 2019 [55], and parts of the publication are reproduced in this chapter.

Chapters 4 and 5 consitute the core of the thesis, as the experimental work done by the author and others is detailed in there, as well as applications of the theory from the previous chapters.

Chapter 4 describes the OpticalDepth campaign at the OMEGA laser facility, where the effects of the plasma geometry on its X-ray spectra were studied for dot targets in HED conditions. The chapter describes the experimental set-up, as well as the experimental results and modelling techniques used in the simulations. The robustness of the platform and its capabilities to benchmark atomic models are also detailed. The results from this chapter were published in Physics of Plasmas in 2019 [56], and so reproductions of the publication are included in the text.

Chapter 5 presents the results from the application of the tools developed in the previous chapters to the previously described Dot Spectroscopy campaign at NIF. The full analysis method is presented, showing how this work can be used to diagnose the ion density inside the hohlraum as well as the temperature. The obtained results are then compared with hydrodynamic predictions, and an analysis of the importance of accounting for the plasma geometry is shown.

Finally, Chapter 6 contains a summary of the work presented and the main con- 
clusions to be extracted from it. Future work relevant to the topics described in the previous chapters is also described.

\subsection{Author contributions}

HED and ICF experiments are, by nature, the result of sizeable collaborations that are made possible by the collaborative work of several people and institutions. The particular contributions of the author to the work in this thesis are outlined in detail here, as well as specific and crucial contributions from collaborators.

All the Cretin simulations presented in this thesis are due to the author. However, they rely heavily in the work of the code developer, H. A. Scott. His assistance with the usage of the code, as well as that of H. P. Le has been invaluable. The section about the code Cretin included in Chapter 1, is also the result of discussions between the author and H. A. Scott.

The calculations and numerical results presented in Chapter 2 are the author's work, as well as the mathematical method to measure the ion density of cylindrical plasmas described in Chapter 3 , but they are the result of fruitful discussions with D. A. Liedahl, S. J. Rose and J. S. Wark. The idea supporting these calculations, and the theory of the geometric effects on spectra relies on the work by F. M. Kerr, S. J. Rose and J. S. Wark.

Although the author was involved in several experiments, the OpticalDepth-17A experiment, whose results are described in Chapter 4, was performed at the start of 2017 and therefore the author did not participate in the preparation of the experiment. It includes contributions from the authors in reference [56]. Special acknowledgement should go to L. C. Jarrott, who was the Principal Investigator (PI) of the experiment, and whose work improved greatly the experimental set-up. Throughout the course of the past four years, input from E. V. Marley and R. F. Heeter on the experimental 
side of the analysis, as well as from D. A. Liedahl and M. B. Schneider was also absolutely crucial to obtaining the results presented here.

The data processing of this experiment was performed solely by the author, as well as the analysis of the X-Ray Pinhole Cameras and the Multipurpose Spectrometers, although the routines are deeply based on those by L. C. Jarrott. While the temperature calculations in Chapter 4 were done by the author, those in Chapter 5 were performed by D. A. Liedahl. The operation of the genetic algorithm written by the author is also based on the code developed by D. A. Liedahl.

The Dot Spectroscopy experiments performed at $\mathrm{NIF}$ were the result of a long campaign that involved many brilliant researchers and whose PI was M. A. Barrios. The author was not involved in these experiments. The analysis of the X-ray imaging was performed by M. A. Barrios and the hydrodynamic simulations with the code HYDRA [57] were developed and run by O. Jones. The spectral analysis is due to the author, with the help of D. A. Liedahl, M. B. Schneider, S. J. Rose and J. S. Wark. Discussions from many more researchers at LLNL and the University of Oxford also provided useful ideas and feedback and helped in the process that led to the results presented in this thesis. 


\section{Chapter 2}

\section{Radiative transfer and plasma}

\section{geometry}

This chapter is dedicated to analysing radiative transfer in plasmas. This phenomenom can be characterised by the combination of two main effects, the modification of the radiation field by the plasma, and the change in the plasma conditions as a result of the presence of the radiation field. These two effects are discussed in Section 2.1, together with some approximations to the corresponding equations, which will be useful for the following chapters. The approach followed in this chapter is that given by Mihalas [58].

The effects of the plasma geometry on its X-ray emission appear then as a consequence of solving the radiative transfer equation. In particular, this thesis focuses on the enhancement of optically thick lines in non-spherical plasmas, an effect that drives the experiments described in Chapter 4. Section 2.2 describes this effect and the previous work done on it. This section is part of a publication in Physics of Plasmas [56]

In Section 2.3, the radiative transfer equation is solved for several particular geometries and the results are discussed in order to illustrate these geometric effects. 
The content of Section 2.3 was published in its entirety in Journal of Quantitative Spectroscopy and Radiative Transfer [54, with the exception of the cylindrical case, which is an excerpt of a publication in High Energy Density Physics [55].

\subsection{The radiative transfer equation}

Consider a beam of radiation with specific intensity $I$ travelling in a direction $\mathbf{n}$ and propagating into a solid angle $d \omega$. The amount of energy absorbed by the plasma in a differential volumen element with area $d S$ and length $d s$ normal to $\mathbf{n}$ at the point $\mathbf{r}$; within a frequency interval $d \nu$ in a time $d t$ is given by

$$
\delta E=I(\mathbf{n}, \mathbf{r}, \nu, t) \kappa(\mathbf{n}, \mathbf{r}, \nu, t) d S d s d \omega d \nu d t
$$

where $\kappa$ is defined as the absorption coefficient or opacity and has units of $\mathrm{cm}^{-1}$ in the cgs system ( $I$ has units of $\mathrm{erg} / \mathrm{Hz} / \mathrm{sr} / \mathrm{s} / \mathrm{cm}^{2}$ ).

In a similar way, the energy emitted by the same volume element into a solid angle $d \omega$ within a frequency interval $d \nu$ in a time $d t$ is given by

$$
\delta E=\eta(\mathbf{n}, \mathbf{r}, \nu, t) d S d s d \omega d \nu d t
$$

where $\eta$ is defined as the emission coefficient or emissivity. In the cgs system, $\eta$ has units of $\mathrm{erg} / \mathrm{Hz} / \mathrm{sr} / \mathrm{s} / \mathrm{cm}^{3}$.

With these definitions it is now possible to write the total energy gain for a radiation beam with intensity $I$ after travelling through the given volume element, as equal to the energy emitted by the plasma minus the energy it absorbs.

$$
\begin{aligned}
{[I(\mathbf{n}, \mathbf{r}+\Delta \mathbf{r}, \nu, t+\Delta t)-} & I(\mathbf{n}, \mathbf{r}, \nu, t)] d S d \omega d \nu d t= \\
& {[\eta(\mathbf{n}, \mathbf{r}, \nu, t)-\kappa(\mathbf{n}, \mathbf{r}, \nu, t) I(\mathbf{n}, \mathbf{r}, \nu, t)] d S d s d \omega d \nu d t }
\end{aligned}
$$


The change in the intensity $I$ can also be indicated using its partial derivatives as

$$
\begin{aligned}
I(\mathbf{n}, \mathbf{r}+\Delta \mathbf{r}, \nu, t+\Delta t)=I(\mathbf{n}, \mathbf{r}, \nu, t)+ & {\left[\left(\frac{\partial I}{\partial t}\right) d t+\left(\frac{\partial I}{\partial s}\right) d s\right]=} \\
& I(\mathbf{n}, \mathbf{r}, \nu, t)+\left[\left(\frac{\partial I}{\partial t}\right) c^{-1}+\left(\frac{\partial I}{\partial s}\right)\right] d s,
\end{aligned}
$$

where $c$ is the speed of light and it has been taken into account that $d s=c d t$. Joining Equations 2.3 and 2.4 and expressing the derivative along the ray path in terms of the gradient $(\partial / \partial s=\mathbf{n} \cdot \nabla)$ the following equation is obtained:

$$
\left[\left(\frac{\partial}{\partial t}\right) c^{-1}+\mathbf{n} \cdot \nabla\right] I(\mathbf{n}, \mathbf{r}, \nu, t)=\eta(\mathbf{n}, \mathbf{r}, \nu, t)-\kappa(\mathbf{n}, \mathbf{r}, \nu, t) I(\mathbf{n}, \mathbf{r}, \nu, t)
$$

This is the completely general form of the radiative transfer equation.

\subsubsection{Radiation coupling to atomic kinetics}

Equation 2.5 describes how the intensity of a radiation field changes when travelling through an plasma as a function of the conditions of the system (its emissivity and opacity). However, the plasma itself is also disturbed by the presence of radiation, as generally, this radiation will be absorbed and re-emitted while travelling through the plasma, thus altering the population of the different atomic states.

To study this effect, consider the general rate equation for an atomic state $u$ with a population $n_{u}$ which can decay to a lower energy state $l$ with a population $n_{l}$. The density of atoms in state $u$ will depend on the spontaneous radiative emission (proportional to the Einstein coefficient $\left.A_{u l}\right)$ and the stimulated emission and absorption of radiation (given by the Einstein coefficients $B_{u l}$ and $B_{l u}$ respectively). It also depends on non-radiative processes and radiative processes other than the $u \rightarrow l$ transitions, 
which here are denoted by $C$. The rate equation then has the form

$$
\frac{d n_{u}}{d t}=C-n_{u} A_{u l}-n_{u} B_{u l} I(\nu)+n_{l} B_{l u} I(\nu)
$$

where $I(\nu)$ is the intensity of the radiation field at the frequency $\nu$ corresponding to the $u \rightarrow l$ transition.

A complete analysis of the coupling of radiation and matter in a plasma involves solving Equations 2.5 and 2.6 together, which in most cases is computationally expensive. To tackle this problem, many atomic kinetics codes use a escape factor formalism. It consists of averaging the number of photons generated within the plasma with the probability of a photon escaping the system, which is equivalent to including the effect of the radiation field as a modification of the $A_{u l}$ coefficient. This probability is expressed as a escape factor $P_{e}$ as

$$
P_{e} n_{u} A_{u l}=n_{u} A_{u l}+n_{u} B_{u l} I(\nu)-n_{l} B_{l u} I(\nu)
$$

which yields the simplified form of Equation 2.6

$$
\frac{d n_{u}}{d t}=C-P_{e} n_{u} A_{u l}
$$

This equation does not depend on the radiation field anymore, and therefore can be solved independently, provided that the value of $P_{e}$ is known.

The functional form of the escape factors has been a widely discussed issue, and several options have been proposed [59, 60, 61, 62, 63, 64], some of which were later summarised and compared in Phillips et al. [65]. Given that the purpose of the escape factor formalism is to simplify the kinetic calculations for multidimensional systems, the size and geometry of the plasma must be taken into account when calculating the corresponding value of $P_{e}$, regardless of which functional form each particular code 
uses. This is generally done by taking the mean chord as the size of the plasma. The mean chord can be easily calculated by means of the Dirac-Fuchs theorem [66, 67] as

$$
\hat{l}=\frac{4 V}{S}
$$

where $V$ is the total volume of the plasma and $S$ is its surface area.

Given that with a escape factor approach any effects of spatial gradients in 2D or 3D geometries disappear from the calculations and are contained within the escape factor itself, this approach works best when the plasma is uniform or with negligible variations in its internal conditions. For cases when temperature, density or velocity gradients in the plasma are present, care must be taken and it is usually more convenient to work with radiative transfer codes which can solve multidimensional systems (although velocity gradients can be included using Sobolev's escape factors). Although the code Cretin (described in Section 1.2) can work using a escape factor approach, as most atomic kinetics codes do, it has the additional capability to work with multidimensional systems by solving the radiative transfer equation. For the simulations presented in this thesis, the 2-dimensional mode of Cretin [31] was used for solving cylindrical systems.

\subsubsection{1-Dimensional solution of the radiative transfer equa- tion}

For simplicity, for the remainder of this thesis some assumptions will be made to simplify Equation 2.5. Firstly, in the one-dimensional case of radiation travelling in one direction, the three-dimensional components of the equation can be dropped, which yields

$$
\left[\left(\frac{\partial}{\partial t}\right) c^{-1}+\left(\frac{\partial}{\partial z}\right)\right] I(z, \nu, t)=\eta(z, \nu, t)-\kappa(z, \nu, t) I(z, \nu, t) .
$$


Furthermore, it is possible to neglect the time-dependent part of the equation, as long as the temporal variations happen in a time scale $\Delta t$ such that $\Delta t<<\Delta z / c$, where $\Delta z$ is the lengthscale of the plasma under consideration. As an example, for a plasma of size $100 \mu \mathrm{m}$, temporal variations slower than $\sim 0.3 \mathrm{ps}$ can be neglected. Given that the experiments presented in this thesis evolve in the nanosecond scale, it is possible for the purpose of this work to consider the time-independent version of the radiative transfer equation. This can then be written as

$$
\frac{\partial I}{\partial z}=\eta(z, \nu)-\kappa(z, \nu) I(z, \nu)
$$

This first order Ordinary Differential Equation (ODE) can now be easily solved. Dividing Equation 2.11 by $\kappa$ and dropping the explicit $z$ and $\nu$ dependence,

$$
\frac{1}{\kappa} \frac{\partial I}{\partial z}=S-I
$$

where $S=\eta / \kappa$ is called the source function. This is now directly solved by performing the integration

$$
\int_{I_{0}}^{I(z)} \frac{\partial I}{S-I}=\int_{z_{0}}^{z} \kappa \partial z,
$$

where $I_{0}$ is the intensity of the radiation field at the point $z_{0}$. If the plasma is assumed to be uniform, and therefore $S$ and $\kappa$ are independent of the spatial coordinate $z$, Equation 2.13 has the solution

$$
I(z)=I_{0} e^{-\kappa\left(z-z_{0}\right)}+S\left(1-e^{-\kappa\left(z-z_{0}\right)}\right) .
$$

Without loss of generality, $z_{0}$ can be taken as the origin. Now, two interesting results can be derived. First, if the self emission of the plasma is much smaller than the external radiation field for a given frequency (that is, if $S \ll I_{0}$ ), Equation 2.14 can 
be simplified to

$$
I(z)=I_{0} e^{-\kappa z}
$$

which is the widely known Beer-Lambert law for the attenuation of a radiation field travelling through a material [68, 69]. From Equation 2.15, it is also possible to obtain the physical interpretation of the opacity $\kappa$, as the inverse of the mfp of radiation of a given frequency.

Secondly, from Equation 2.14, if no external illumination is shone upon the plasma $\left(I_{0}=0\right)$, the following solution is obtained

$$
I(z)=S\left(1-e^{-\kappa z}\right)
$$

It is now possible to obtain the intensity of the radiation emitted by a plasma in a direction through which the Line Of Sight (LOS) is $\Delta z$ as

$$
I=S\left(1-e^{-\kappa \Delta z}\right)=S\left(1-e^{-\tau}\right)
$$

where $\tau=\kappa \Delta z$ is called the optical depth of the plasma. Recalling that $\kappa$ is the inverse $\mathrm{mfp}$ of the radiation, $\tau$ can be understood as the size of the plasma in units of the $\mathrm{mfp}$. This interpretation introduces an important distinction for radiation of different frequencies based on their optical depth.

If for a given frequency, $\tau>1$, that is the length of the plasma is greater than a mfp at said frequency, the radiation is said to be optically thick, and photons of that frequency are likely to be absorbed before escaping the plasma. If, on the contrary, $\tau<1$ the radiation is said to be optically thin. In the extreme case of optically thin radiation with $\tau \ll 1$, absorption within the plasma can be neglected and the radiation intensity can be approximated from Equation 2.17 as

$$
I=\eta \Delta z
$$




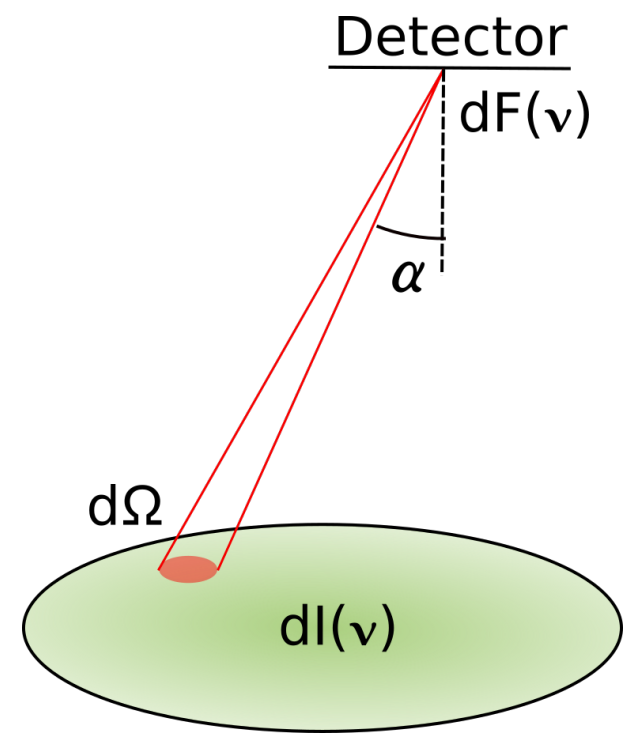

Figure 2.1: Differential element of spectral flux and magnitudes involved in its definition. As the detector is placed further from the plasma, the angle $\alpha$ becomes smaller, until all the rays reaching the detector are parallel and with $\alpha=0$.

From Equation 2.17 it can be seen how for a uniform, steady-state plasma, the intensity of radiation emitted in a given direction only depends on the size of the plasma through the LOS, For this reason, although the mean chord of the plasma is used in escape factor approximations to do the atomic kinetic calculations, it is the LOS that must be used when calculating the spectrum in a given direction.

\subsubsection{Spectral flux}

In experimental conditions it is not possible to measure the radiation intensity, as it is not an observable. The quantity actually measured by detectors is the spectral flux $F$, which is defined as

$$
F(\nu)=\int d \Omega I(\nu) \cos (\alpha)
$$

where $d \Omega$ is the differential solid angle subtended by the source at the detector and $\alpha$ is defined as the angle between a ray leaving the source and the normal vector of the corresponding differential surface element at the detector, as shown in Figure 2.1. $F$ has units of $\mathrm{erg} / \mathrm{Hz} / \mathrm{s} / \mathrm{cm}^{2}$. For practical purposes, hereafter any detectors 
will be assumed to be looking directly at the plasma and sufficiently far from the source so that all the rays reaching the detector are parallel and with $\alpha=0$. It will also be assumed that the detectors cover the whole size of the plasma. Under this assumption, the spectral flux definition is simplified to

$$
F(\nu)=\int \frac{d A}{d^{2}} I(\nu)
$$

where $d A$ is the differential surface area of the source projected in the direction of the detector, and $d$ is the distance from the detector to the source. Combining Equations 2.20 and 2.18 it can immediately be obtained that, in the limit of an optically thin and uniform plasma

$$
F(\nu)_{t h i n}=\int \frac{d A}{d^{2}} \eta \Delta z=\frac{\eta}{d^{2}} V
$$

where $V$ is the total volume of the plasma. Therefore, the spectral flux of optically thin radiation only depends on the total volume of the plasma, and does not change with the direction of observation.

\subsection{Enhancement of optically thick lines}

While from a direct comparison of Equations 2.16 and 2.18 one might expect the presence of opacity to strictly reduce the spectral intensity relative to the optically thin limit, Bhatia and co-workers [70, 71, 72] showed that, under certain conditions, opacity can cause a relative enhancement of the emission of optically thick lines. To explain this phenomenon, consider a small enough plasma, such that it is optically thin for radiation of all frequencies in any direction (Figure 2.2a). In this case, the population of the excited state of any given transition will be determined by the balance between collisional processes and spontaneous radiative decay.

If one of the dimensions of this plasma is now increased, such that radiation from 


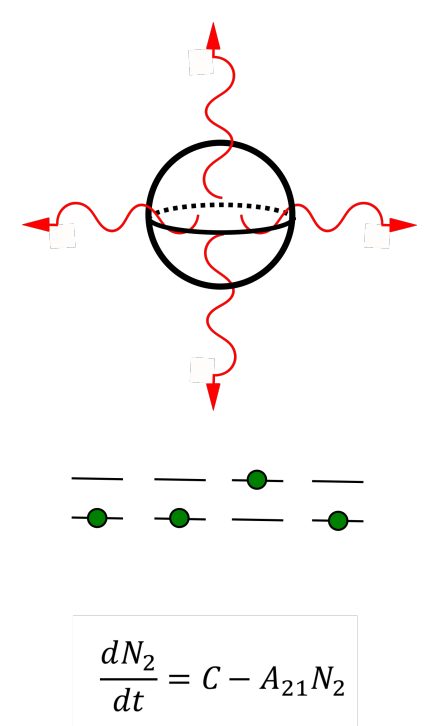

(a)

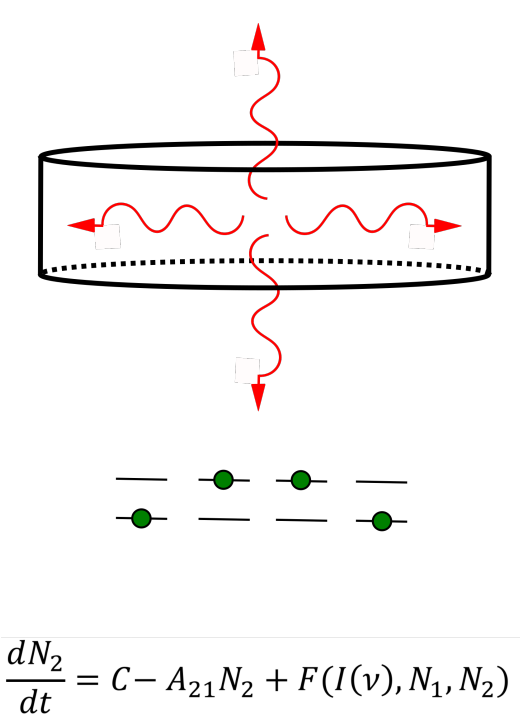

(b)

Figure 2.2: 2.2a) Radiation escaping an optically thin plasma and equation for the population of the excited state of a given transition. (2.2b) Photoabsorption of optically thick radiation in a non-spherical plasma and modification of the atomic populations due to the radiation field within the plasma.

a particular line becomes optically thick when travelling in the modified direction (Figure 2.2b), the population of the excited state of said line will be increased due to the radiation field photopumping the transition. However, for the unmodified dimension, the line remains optically thin, although the population of the excited state has been increased. This results in an enhancement of the emission of optically thick radiation through the 'short' dimension, and a supression through the 'long' dimension of the plasma with respect to the optically thin case. This was shown by Kerr et al. [73, 74, 75] who proved that this effect was related to the ratio between the LOS through the plasma in the direction of the observation and the mean chord of the plasma, with enhancement happening when the LOS is smaller than the mean chord and supression happening in the opposite case.

Kerr's work considered the ratio of two distinct spectral lines in plasmas, uniform in density and temperature but of differing geometries, in astrophysical conditions. The opacity effect exhibits itself in the observed ratio of these two spectral lines that 
traverse the same physical path length (the LOS). Along this length one of the lines is thought to be optically thick, and the other optically thin. Kerr et al. studied the effect of opacity by calculating an intensity ratio for the optically thick line, $f=I_{\text {thick }} / I_{\text {thin }}$, where $I_{\text {thick }}$ is the intensity of this line when calculated including opacity effects, and $I_{\text {thin }}$ is the intensity of the very same line, calculated neglecting such opacity effects both on the populations and in line transfer. following the above explanation, $f$ will be greater than one for mean chords larger than the LOS, whereas if the LOS is greater than the mean chord, $f$ will be less than unity.

The ratio $f$ is not of itself a directly experimentally observable for a given line, as a particular line is either thick or thin. However, whatever the particular species present within a plasma, the ratio $f$ can always be directly related to an experimentally observable ratio between different spectral lines, where one of them is optically thin, simply by multiplying with a known constant - and this is the role played by the second (optically thin) line, referred to above. Therefore, $f$ can be calculated as

$$
f=F_{1}^{\text {thick }} / F_{1}^{\text {thin }}=\frac{F_{1}^{\text {thick }} / F_{2}}{F_{1}^{\text {thin }} / F_{2}},
$$

where $F_{1}$ and $F_{2}$ denote the flux from the optically thick and thin lines respectively. The ratio $F_{1}^{\text {thick }} / F_{2}$ can be measured experimentally, and (as line 2 is always optically thin) the ratio $F_{1}^{\text {thin }} / F_{2}$ is independent of the optical depth, and therefore identical for all directions of observation (all LOS), therefore taking the role of the constant mentioned above.

Given that this effect depends on the mean chord, which is constant for a given geometry, and LOS lengths, which depend on the position of the detector; this effect depends on the relative position of the detector with respect to the plasma and will be referred to as geometric anisotropy. Although it has mostly been studied to characterise the geometry of astrophysical bodies [74, 76], it has also been observed 
in HED experiments [77]. In the past few years, a series of additional experiments to study this effect have been proposed, such as using an Argon gas bag [75] or confining the plasma geometry by burying the targets in aerogel foam [75, 78].

\subsection{Solution in different geometries}

In this section, the radiative transfer for several geometries is studied by explicitly solving Equation 2.20, For simplicity, this work is limited to geometries with a cylindrical symmetry, namely cylinders, hollow hemispherical shells, hollow hemiellipsoidal shells and torii. The spectral flux is obtained for two directions of emission, face-on, or parallel to the axis of symmetry, and side-on, or perpendicular to the axis of symmetry. The ratio between the fluxes in these two directions is also calculated and its use for measuring the frequency resolved opacity of the plasma is discussed. For this section, the explicit frequency dependence, $\kappa(\nu)$, will be included in the equations. It is found that the optimal geometry to use this ratio as an opacity measurement, that is, the geometry on which the face-on and side-on fluxes are most different, is a planar cylinder.

\subsubsection{Cylinder}

First, the simple case of a uniform plasma cylinder, which is discussed in depth in reference [55], is considered. Figure 2.3 shows a schematic drawing of this geometry. Hereafter, the thickness of the cylinder is denoted $H$ and its radius $R$.

As the size of the plasma in the axial direction is constant and equal to $H$ for every point in the emitting surface, the flux emitted in the direction of the cylinder axis (face-on) can be directly obtained from Equations 2.16 and 2.20 as

$$
F_{f o}(\nu)=\frac{\pi R^{2}}{d^{2}} S(\nu)\left(1-e^{-\kappa(\nu) H}\right) .
$$




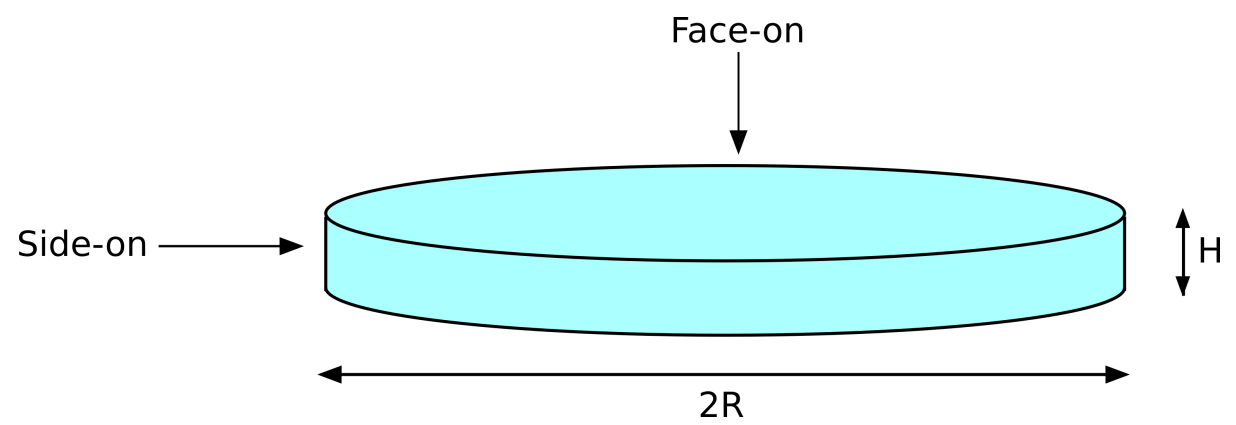

Figure 2.3: Schematic drawing of a cylinder with radius $R$ and thickness $H$.

The emission in the radial direction (side-on) however, is not so simple to calculate. For the side-on view, radiation crossing the cylinder travels a different path length depending on its distance from the centre of the cylinder. Defining an azimuthal angle $\phi$ as indicated in Figure 2.4, it is obtained that a ray crossing the cylinder travels a distance $2 R \sin \phi$ inside the plasma, and the radiation leaves the plasma at an angle $\pi / 2-\phi$. Therefore, for a given value of the azimuthal angle between $\phi$ and $\phi+d \phi$, the differential flux measured for that surface element is given by

$$
d F_{s o}(\nu)=\frac{H R \sin \phi}{d^{2}} S(\nu)\left(1-e^{-\kappa(\nu) 2 R \sin \phi}\right) d \phi .
$$

where $H R \sin \phi d \phi$ is the projected surface area in the direction of the detector. And so, the total flux in the side-on direction is given by

$$
F_{\text {so }}(\nu)=\frac{H R}{d^{2}} S(\nu) \int_{0}^{\pi}\left(1-e^{-2 \kappa(\nu) R \sin \phi}\right) \sin \phi d \phi,
$$

which can be written explicitly as

$$
F_{s o}(\nu)=\frac{2 H R}{d^{2}} S(\nu)\left[1-\frac{\pi}{2}\left(L_{-1}(2 \kappa(\nu) R)-I_{1}(2 \kappa(\nu) R)\right)\right]
$$

where $L_{n}$ is the modified Struve function of order $n$ and $I_{n}$ is the $n$-th order modified Bessel function of the first kind [79]. It is interesting to note here than in the opti- 


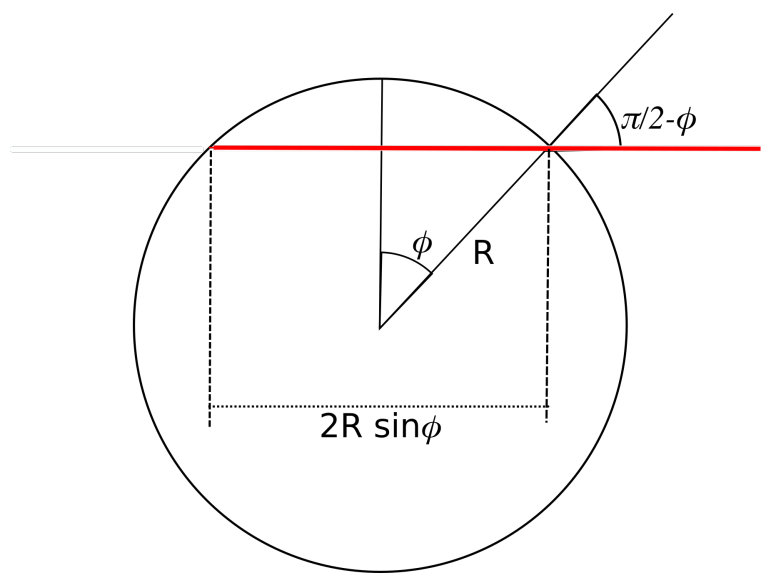

Figure 2.4: Top view of a cylinder showing one ray (red) travelling to the side-on detector. Rays span a range of chord lengths from 0 to $2 R$. If the azimuthal angle $\phi$ is defined as shown, a photon travels a distance $2 R \sin \phi$ before leaving the plasma, and exits with an angle $\pi / 2-\phi$ with respect to the surface normal vector.

cally thin limit $(\kappa R \rightarrow 0)$, the factor in square brackets in Equation 2.26 reduces to $\left[1-\exp \left(\frac{\pi}{2} \kappa R\right)\right]$. This is equivalent to assuming that all photons travel the average path length before escaping the plasma (the mean chord for the side-on view of a cylinder is $\pi R / 2$ ). However, this is not valid when the conditions are not entirely optically thin.

Working with Equation 2.26 is not computationally (or analytically) easy, given the complicated functions inside the brackets. One possible approach is to approximate the exact solution by a function of the form $(1-\exp [-a \kappa R])$, by choosing a value of $a$ such that it minimises the differences with the exact solution over a wide range of $\kappa R$ values [55, 80]. In this case, a maximum value for $\kappa R$ of 10 was chosen to ensure convergence of the calculations, and the optimal value of $a$ was found to be 1.45. Using this approximation, there is less than $5 \%$ error for all values of $\kappa R$ in this range. The difference between both functions decreases as $\kappa R$ increases and both functions tend asymptotically to 1 . This difference becomes less than $1 \%$ for $\kappa R \sim 5$ and for $\kappa R=10$ its value is only $\sim 0.25 \%$. Both the approximation and the exact solution are shown in Figure 2.5. 


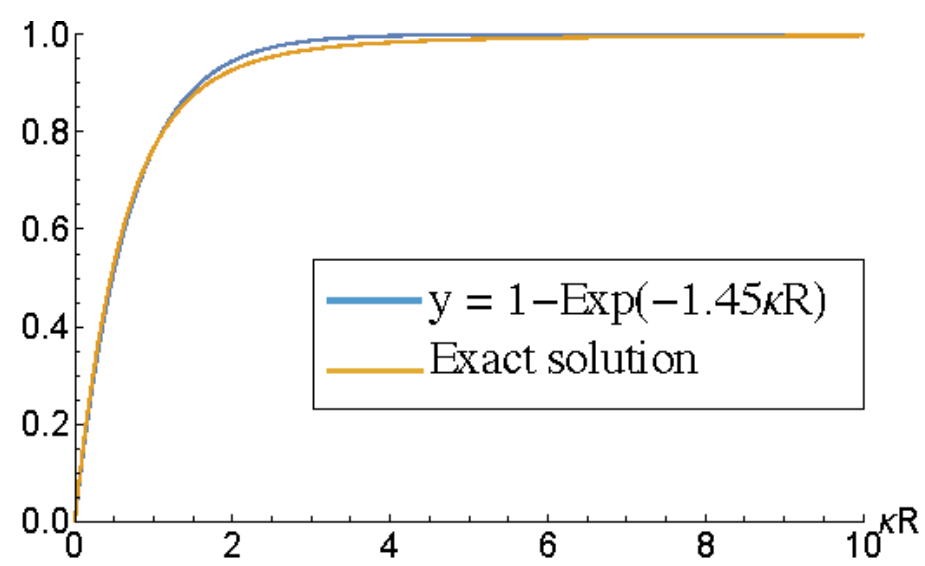

Figure 2.5: Comparison between the exact value of the brackets in Equation 2.26, and the approximation used in this thesis.

With this approximation, Equation 2.26 is now written as

$$
F_{s o}(\nu)=\frac{2 H R}{d^{2}} S(\nu)\left(1-e^{-1.45 \kappa(\nu) R}\right)
$$

To simplify the notation, the aspect ratio of the cylinder $a=H / R$ and the optical depth parameter $\gamma(\nu)=\kappa(\nu) R$ are introduced. It is now possible to write the ratio between the face-on and side-on flux for a cylinder by dividing Equations 2.23 and 2.27 as

$$
\frac{F_{f o}(\nu)}{F_{s o}(\nu)}=\frac{\pi}{2 a} \cdot \frac{1-e^{-a \gamma(\nu)}}{1-e^{-1.45 \gamma(\nu)}}
$$

Figure 2.6 shows a comparison between the result from Equation 2.28 and the exact solution (which uses Equation 2.26) for a value of $a=0.2$. To understand the potential use of this expression, consider an experiment in which the aspect ratio $a$ is known and the face-on and side-on spectra are measured. Equation 2.28 allows the use of their ratio as a function of frequency to obtain $\gamma(\nu)$, and thereby the frequency dependent opacity $(\gamma(\nu)=\kappa(\nu) R)$. The accuracy of the measurement will depend on the limit

$$
\frac{F_{f o} / F_{s o}(\gamma(\nu) \rightarrow \infty)}{F_{f o} / F_{s o}(\gamma(\nu) \rightarrow 0)}=\frac{1.45}{a}
$$

as when this ratio is greater, the flux ratio is more sensitive to differences in opacity. 


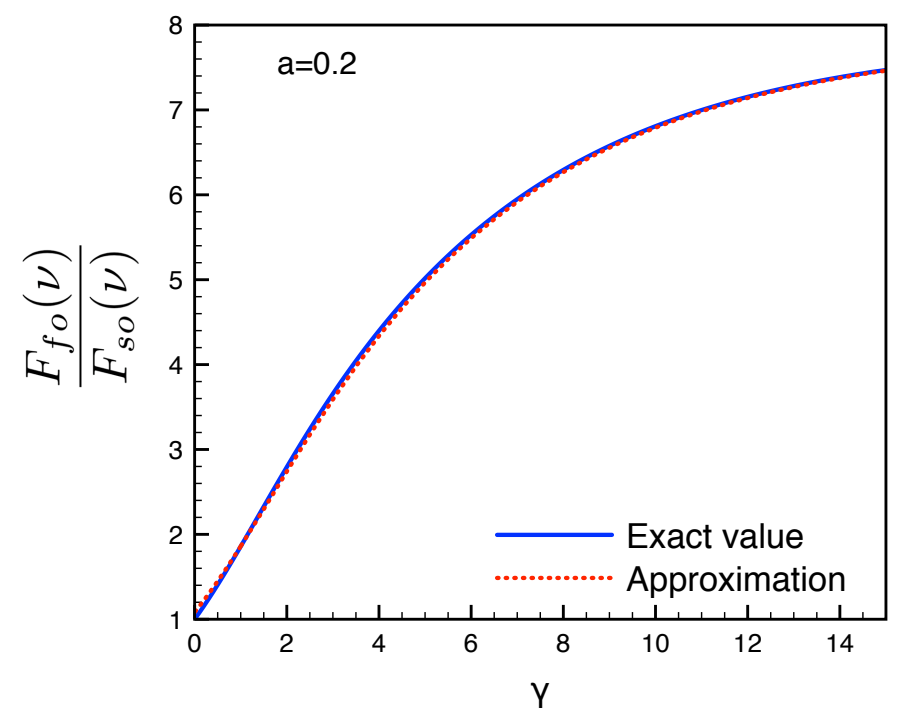

Figure 2.6: Ratio between the face-on and side-on flux for a cylinder (given by Equations 2.23, 2.27 and 2.28) as a function of $\gamma(\nu)$ for an aspect ratio $a=0.2 . \gamma(\nu)$ can be obtained from a measurement of this ratio. In the limit of high values of $\gamma(\nu)$ this ratio tends to the ratio between the emitting surface areas in the face-on and side-on directions. For optically thin radiation, this ratio is 1, as radiation escapes the plasma isotropically. The exact solution and the result from the approximation used in this thesis are shown for comparison.

From Equation 2.29 it follows that in cylindrical geometries, the highest sensitivity with this method, that is, the greatest differences between the face-on and the side-on flux are obtained for low aspect ratios $(H \ll R)$.

\subsubsection{Hemispherical shell}

Consider now the case of a hemispherical shell of thickness $\Delta r$ consisting of a plasma of opacity $\kappa(\nu)$. The hemisphere lies on the XY plane (Figure 2.7). The flux emitted in the $z$ direction (face-on) is

$$
F_{f o}(\nu)=\frac{1}{d^{2}} \int S(\nu)\left(1-e^{-\kappa(\nu) \Delta r^{\prime}}\right) d A
$$

where $\Delta r^{\prime}$ is the path traveled in the direction of emission for a given ray. Under the assumption that $\Delta r$ is small with respect to the radius of the sphere, it is possible 


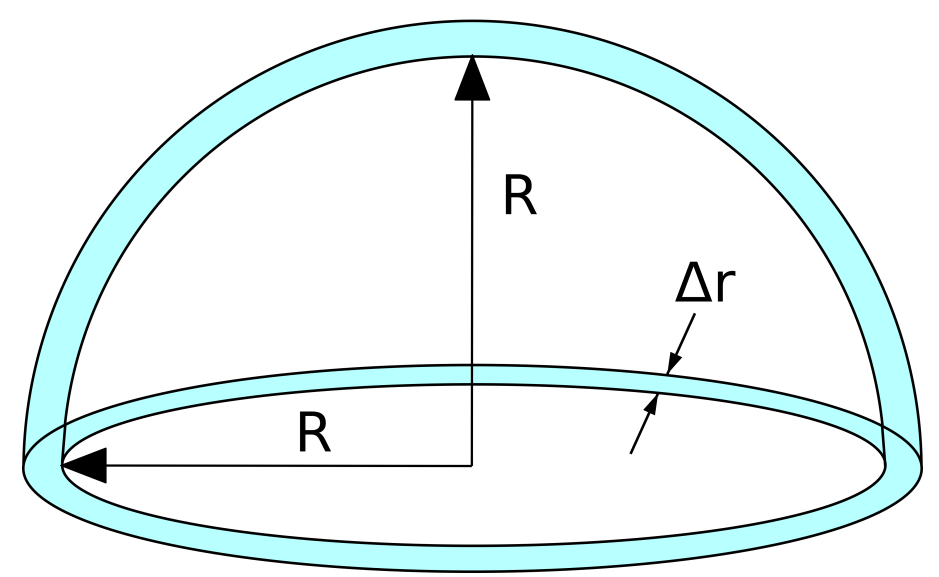

Figure 2.7: Schematic drawing of a hemispherical shell with radius $R$ and thickness $\Delta r$.

to take $\Delta r^{\prime}=\Delta r / \cos (\theta)$, where $\theta$ is the polar angle. Defining in this case $\gamma(\nu)=$ $\kappa(\nu) \Delta r$, this equation has the solution

$$
F_{f o}(\nu)=\frac{\pi R^{2}}{d^{2}} S(\nu)\left[1-e^{-\gamma(\nu)}(1-\gamma(\nu))-\gamma(\nu)^{2} E_{1}(\gamma(\nu))\right]
$$

where $E_{1}(x)$ is the first order exponential integral function [79].

The term in square brackets in Equation 2.31 can be approximated by a function of the form $\left(1-e^{-b \gamma(\nu)}\right)$, similarly as in the previous section. The value of $b$ that minimises the difference is 1.5, which yields a maximum error of $\sim 30 \%$ for $\gamma(\nu) \rightarrow 0$, quickly dropping below $5 \%$ for $\gamma(\nu)>0.45$. A comparison between the exact value and the exponential approximation is shown in Figure 2.8. With this approximation

$$
F_{f o}(\nu)=\frac{\pi R^{2}}{d^{2}} S(\nu)(1-\exp [-1.5 \gamma(\nu)])
$$

Thanks to the symmetry of the system, the side-on emission is identical, doubling the LOS and halving the surface area

$$
F_{s o}(\nu)=\frac{\pi R^{2}}{2 d^{2}} S(\nu)\left[1-e^{-2 \gamma(\nu)}(1-2 \gamma(\nu))-4 \gamma(\nu)^{2} E_{1}(2 \gamma(\nu))\right]
$$




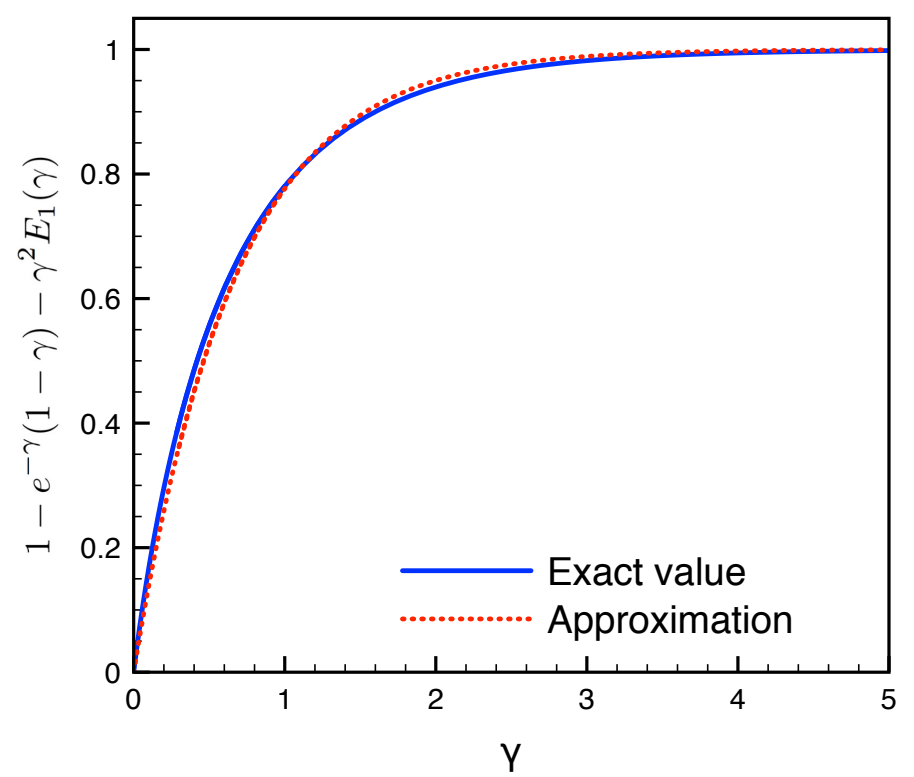

Figure 2.8: Comparison between the exact value of the square brackets in Equation 2.31 and the approximation given in Equation 2.32. The limits in the x-axis have been chosen so that the calculations converge, and the asymptotic behaviour can be observed.

And therefore, using the same approximation, the side-on flux is given by

$$
F_{s o}(\nu)=\frac{\pi R^{2}}{2 d^{2}} S(\nu)(1-\exp [-3 \gamma(\nu)])
$$

The comparison between the flux from both views as a function of $\gamma(\nu)=\kappa(\nu) \Delta r$ is shown in Figure 2.9. For high values of $\gamma$ this ratio tends asymptotically to 2, which is the ratio between the surface areas seen by the face-on and side-on detectors. For optically thin radiation, this ratio is 1 , as opacity effects are negligible and radiation escapes the plasma isotropically.

In this particular geometry, the range of the face-on to side-on ratio is a factor of 2. Given that this range determines the sensitivity of the opacity measurements obtained from the flux ratio, using a cylinder with $H / R<0.725$ is preferable to a spherical shell (see Equation 2.29). 


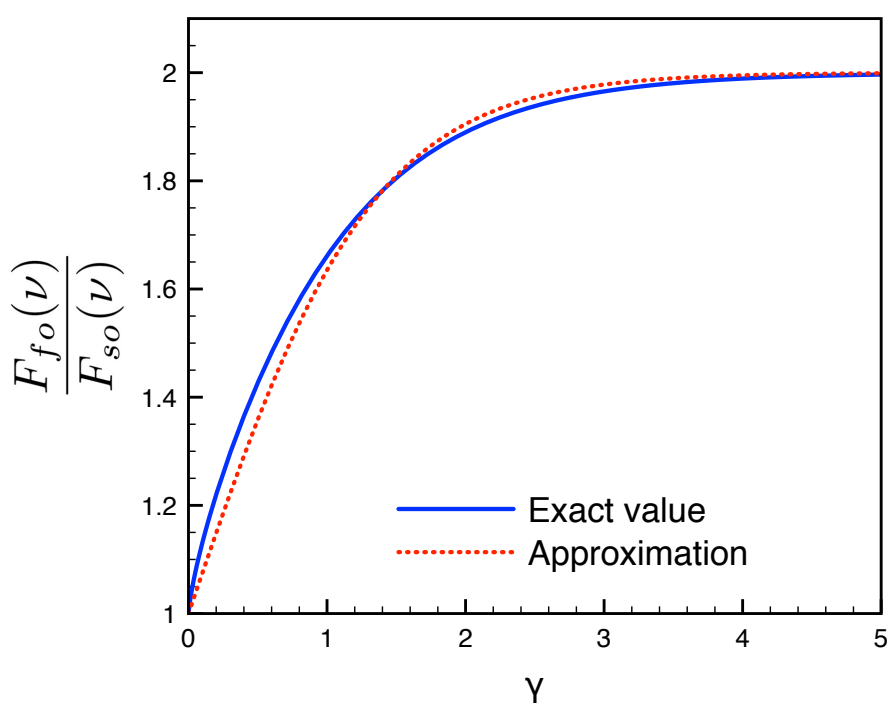

Figure 2.9: Ratio between the face-on and side-on flux for a hemispherical shell as a function of $\gamma(\nu)$.

\subsubsection{Hemi-ellipsoidal shell}

The case of a symmetric hemi-ellipsoidal shell as shown in Figure 2.10 is now considered. The thickness of the shell is $\Delta r$, and $\gamma(\nu)=\kappa(\nu) \Delta r$ as in the spherical case. The aspect ratio of the ellipsoid is denoted $a=R_{2} / R_{1} . \Delta r$ will also be assumed to be small compared with the two main radii of the hemi-ellipsoid. Proceeding as in the hemi-spherical case, the flux emitted in the face-on direction is given by

$$
F_{f o}(\nu)=\frac{2 \pi R_{1}^{2}}{d^{2}} S(\nu) \int_{0}^{\pi / 2} \sin (\theta) \cos (\theta)\left(1-e^{-\gamma(\nu) \sin (\theta)\left(1+\frac{a}{\tan ^{2}(\theta)}\right)}\right) d \theta
$$

which hereafter is written as

$$
F_{f o}(\nu)=\frac{\pi R_{1}^{2}}{d^{2}} S(\nu) G_{e l l}^{f o}(a, \gamma(\nu))
$$

where $G_{\text {ell }}^{\text {fo }}$ asymptotically tends to 1 for high values of $\gamma(\nu)$.

The function $G_{\text {ell }}^{\text {fo }}$ cannot be expressed as an analytical function, and must be calculated numerically. In a similar way to the previous section, it is also approximated 


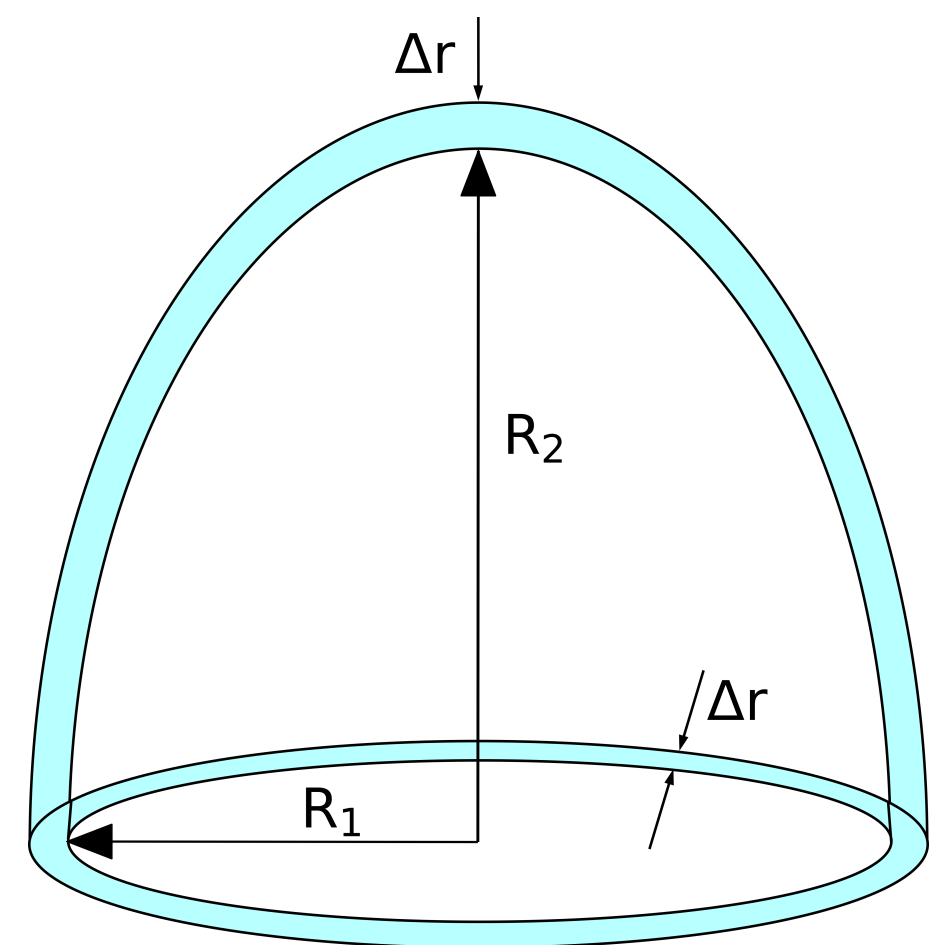

Figure 2.10: Schematic drawing of a symmetric hemi-ellipsoidal shell with radii $R_{1}$ and $R_{2}=a R_{1}$; and thickness $\Delta r$.

by a function of the form $\left(1-\exp \left[-b_{\text {ell }}^{f o}(a) \gamma\right]\right)$, where here $b_{\text {ell }}^{\text {fo }}$ depends on $a$. The values of the function $b_{\text {ell }}^{f o}$ that minimise the difference between the true value and the approximation for different values of $a$, and all $\gamma$ values are shown in Figure 2.11. A polynomial fit to these values is

$$
b_{\text {ell }}^{f o}(a)=-0.08 a^{2}+0.82 a+0.75 \text {. }
$$

And therefore, it is possible to approximate the face-on flux of a symmetric hemiellipsoid as

$$
F_{f o}(\nu)=\frac{\pi R_{1}^{2}}{d^{2}} S(\nu)\left(1-e^{-b_{e l l}^{f o}(a) \gamma(\nu)}\right)
$$

An example of the comparison between the exact value and the approximation is shown in Figure 2.12. This example corresponds to an aspect ratio $a=0.3$. 


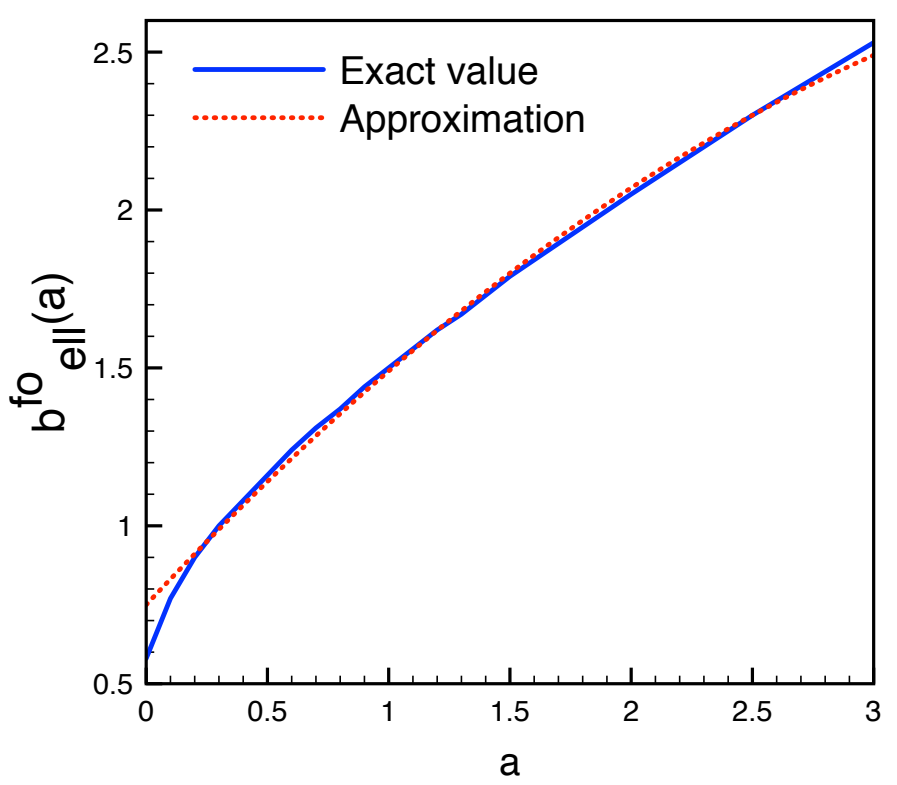

Figure 2.11: Value of $b_{\text {ell }}^{f o}(a)$ such that $1-\exp \left[-b_{\text {ell }}^{f o}(a) \gamma\right]$ is the best fit to the exact solution for the face-on flux of a hemi-ellipsoidal plasma with aspect ratio $R_{2} / R_{1}=a$. The polynomial fit shown is valid for values of $a$ between 0.3 and 3 .

The side-on emission of the hemi-ellipsoid is given by

$$
\begin{aligned}
F_{\text {so }}(\nu)=\frac{a R_{1}^{2}}{d^{2}} S(\nu) \int_{-\pi / 2}^{\pi / 2} \cos (\phi) d \phi & \\
& \int_{0}^{\pi / 2} \cos ^{2}(\theta)\left(1-e^{-2 \gamma(\nu) \cos (\theta) / \cos (\phi)\left(1+\frac{\tan ^{2}(\theta)}{a}\right)}\right) d \theta .
\end{aligned}
$$

which can also be written as

$$
F_{\text {so }}(\nu)=\frac{\pi a R_{1}^{2}}{2 d^{2}} S(\nu) G_{e l l}^{s o}(a, \gamma(\nu))
$$

where $G_{\text {ell }}^{\text {so }}(a, \gamma(\nu))$ asymptotically tends to 1 for high values of $\gamma(\nu)$. Using the same approach as previously, Equation 2.40 becomes

$$
F_{s o}=\frac{\pi a R_{1}^{2}}{2} \cdot \frac{S(\nu)}{d^{2}}\left(1-e^{-2 b_{e l l}^{s o}(a) \gamma(\nu)}\right) .
$$




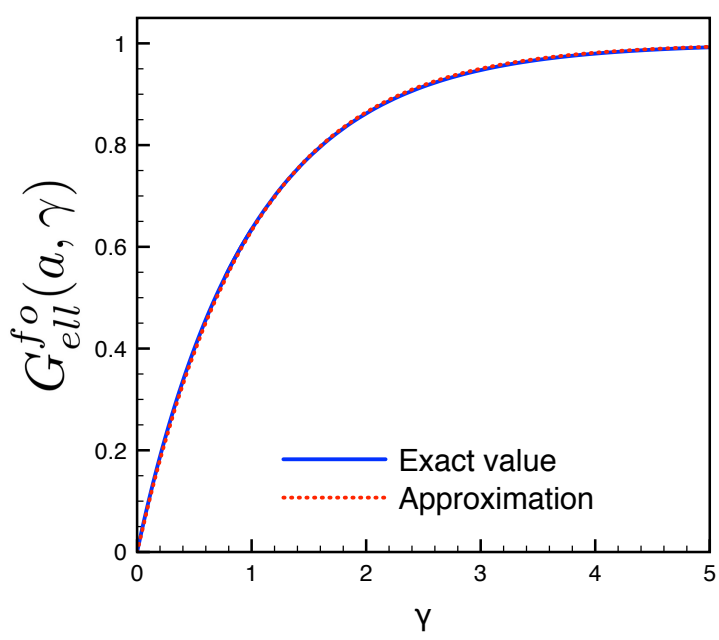

(a)

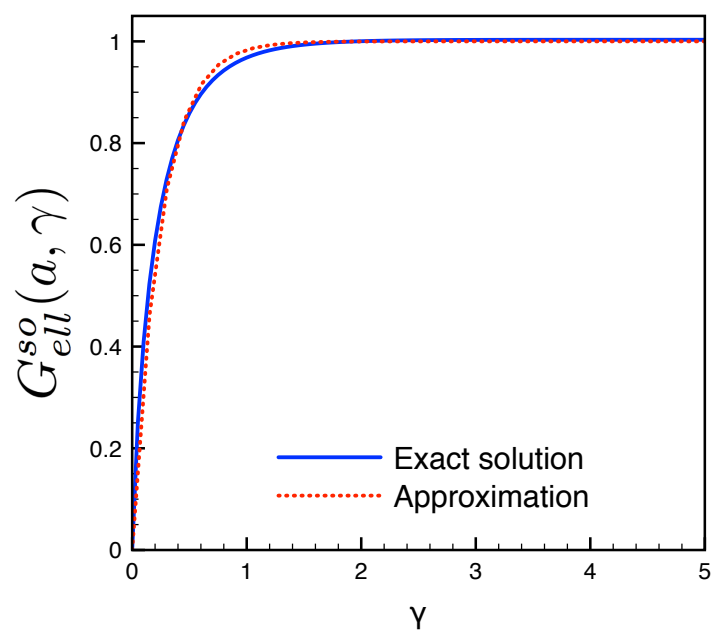

(b)

Figure 2.12: 2.12 Comparison between the exact value for $G_{\text {ell }}^{f o}(a, \gamma)$ and the function $1-e^{-b_{\text {ell }}^{f o}(a) \gamma}$. 2.12b Comparison between the exact value for $G_{\text {ell }}^{\text {so }}(a, \gamma)$ and the function $1-e^{-b_{\text {ell }}^{\text {so }}(a) \gamma}$. The aspect ratio in both cases is $a=0.3$.

where, as before, $b_{\text {ell }}^{\text {so }}(a)$ is approximated as

$$
b_{\text {ell }}^{\text {so }}(a)=-0.013 / a^{2}+0.298 / a+1.191 .
$$

The functions $G_{e l l}^{f o}(a, \gamma(\nu)), G_{e l l}^{s o}(a, \gamma(\nu))$ and their corresponding approximations are shown in Figure 2.12 for a value of $a=0.3$.

The ratio of face-on to side-on flux from in a hemi-ellipsoidal shell depends only on $\gamma(\nu)$ and $a$. As previously discussed, the bigger the range covered by this ratio, that is, the greater the value of

$$
\frac{F_{f o} / F_{s o}(a, \gamma(\nu) \rightarrow \infty)}{F_{f o} / F_{s o}(a, \gamma(\nu) \rightarrow 0)}
$$

the more precise an opacity measurement will be. This ratio is shown in Figure 2.13 , where it can be seen how for small values of $a$ (when the geometry becomes planar and more similar to a cylinder), the ratio in Equation 2.43 and therefore the accuracy of an opacity measurement, is greatest. 


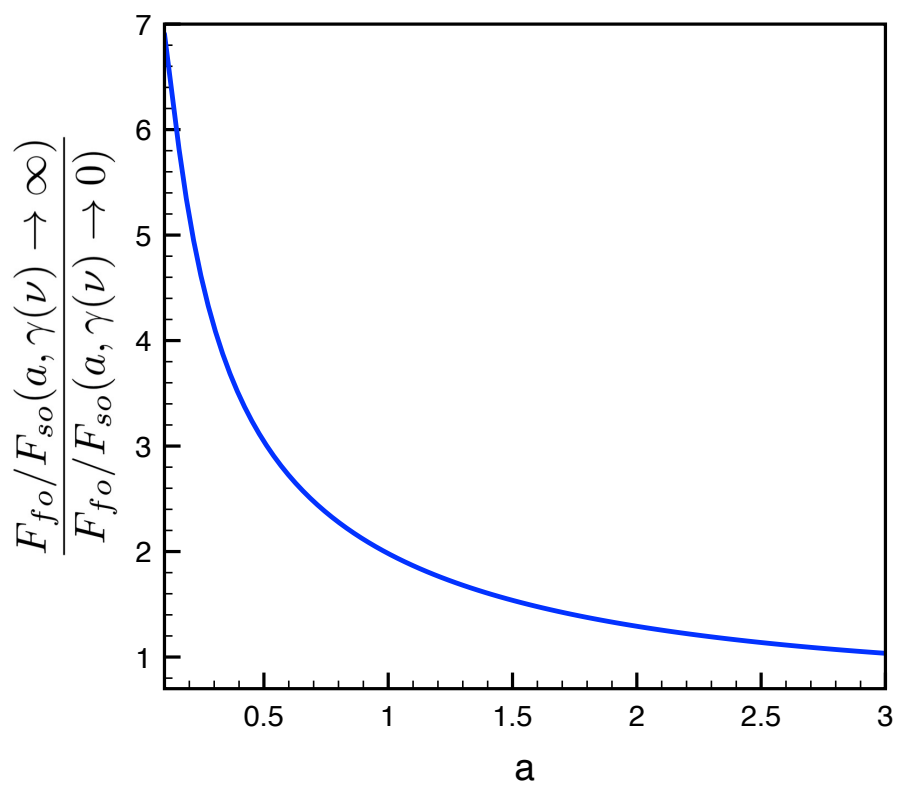

Figure 2.13: Ratio between the face-on/side-on flux for optically thick to optically thin radiation in a symmetric hemi-ellipsoidal plasma as a function of its aspect ratio $a=R_{2} / R_{1}$. The greatest sensitivity is obtained for low aspect ratios, that is, when the hemi-ellipsoid becomes planar.

\subsubsection{Torus}

The final geometry considered in this chapter is a torus where the radius of revolution is denoted $R$ and the radius of the revolving circle is denoted $r$. This is schematically shown in Figure 2.14. In this section, it will be assumed that $R \geq r$.

The face-on emission (in the direction of the axis) can be considered as the side-on emission of a series of differential cylinders with thickness $R d \theta$. This was described in Section 2.3.1, and has the form [55]

$$
F_{f o}(\nu)=\frac{4 \pi a r^{2}}{d^{2}} S(\nu)\left[1-\frac{\pi}{2}\left(L_{-1}(2 \gamma(\nu))-I_{1}(2 \gamma(\nu))\right)\right]
$$

where for the toroidal geometry, $\gamma(\nu)$ is defined as $\kappa(\nu) r$, and $a=R / r$. So, proceeding as in Section 2.3.1, the face-on flux of a torus is given to a good approximation by

$$
F_{f o}=\frac{4 \pi a r^{2}}{d^{2}} S(\nu)\left[1-e^{-1.45 \gamma(\nu)}\right]
$$




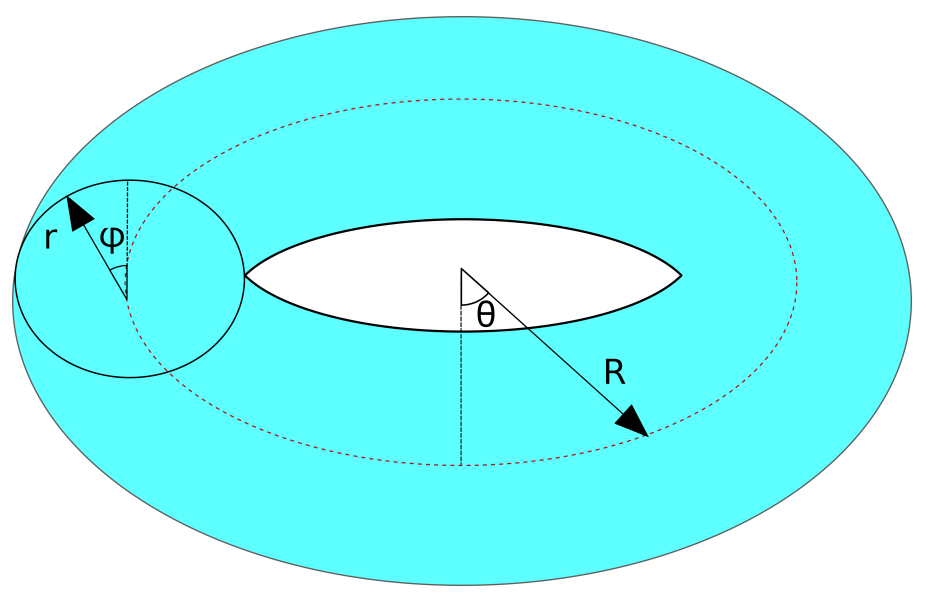

Figure 2.14: Schematic diagram of a plasma torus, where $r$ is the radius of the revolving circle, and $R$ is the revolving radius. The azimuthal and polar angles $\phi$ and $\theta$ are defined as shown.

The side-on emission of a torus has two different components: rays leaving the torus through its inner hole and then reentering before escaping, and rays that do not go through the hole. The paths that these two different kinds of rays travel are given by

$$
\Delta x_{h}=2\left[(R+r \sin \phi) \cos (\theta)-\sqrt{(R+r \sin \phi)^{2} \cos ^{2}(\theta)-4 R r \sin \phi}\right],
$$

and

$$
\Delta x_{n h}=2(R+r \sin \phi) \cos (\theta)
$$

where $\Delta x_{h}$ corresponds to rays that go through the central hole and $\Delta x_{n h}$ corresponds to rays that do not. The total side-on flux is the sum of these two contributions, which 


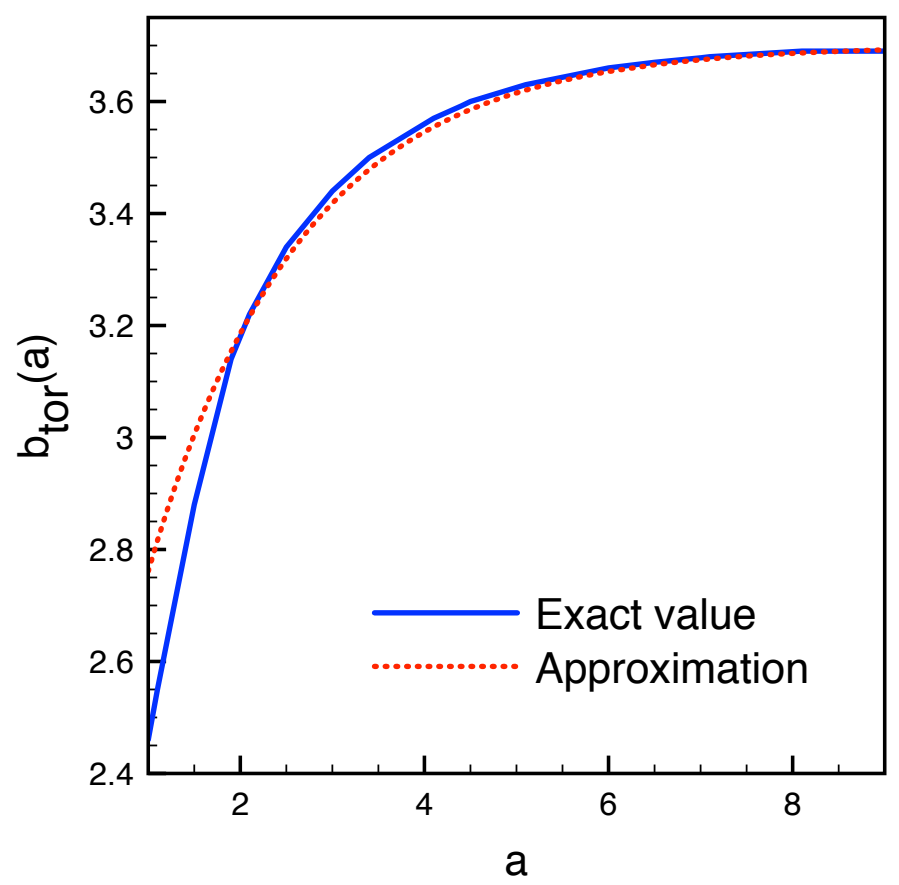

Figure 2.15: Value of $b_{\text {tor }}(a)$, such that the function $1-\exp \left[-b_{t o r}(a) \gamma\right]$ minimises the differences with the exact solution for the side-on emission of a plasma torus with aspect ratio $a=R / r$.

is

$$
\begin{gathered}
F_{s o}(\nu)=\frac{4 r^{2}}{d^{2}} S(\nu)\left[\int_{0}^{\pi / 2}(a+\sin \phi) \sin \phi d \phi\right. \\
\left(\int_{0}^{\arcsin \left(\frac{a-\sin \phi}{a+\sin \phi}\right)}\left[1-e^{-2 \gamma(\nu)\left[(a+\sin \phi) \cos \theta-\sqrt{(a+\sin \phi)^{2} \cos ^{2} \theta-4 a \sin \phi}\right]}\right] \cos \theta d \theta+\right. \\
\left.\left.\int_{\arcsin \left(\frac{a-\sin \phi}{a+\sin \phi}\right)}^{\pi / 2}\left[1-e^{-2 \gamma(\nu)(a+\sin \phi) \cos \theta}\right] \cos \theta d \theta\right)\right],
\end{gathered}
$$

as usual, this can be written as

$$
F_{s o}(\nu)=\frac{(4 a+\pi) r^{2}}{d^{2}} S(\nu) G_{t o r}^{s o}(a, \gamma(\nu))
$$

where $G_{t o r}^{s o}$ asymptotically tends to 1 for high values of $\gamma(\nu)$, and the first factor is the solid angle subtended by the total project area in the direction of the detector.

The function $G_{t o r}^{s o}$ can be approximated as $\left(1-\exp \left[-b_{t o r}(a) \gamma(\nu)\right]\right)$. The values of 


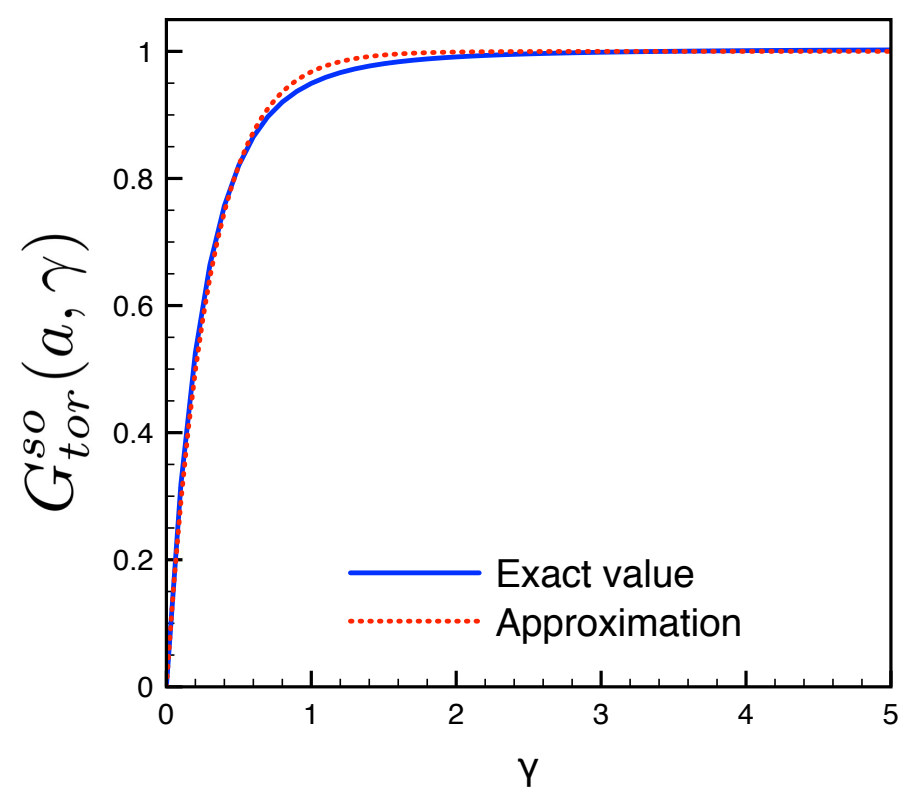

Figure 2.16: Comparison between the exact value for the side-on emission of a torus, given by the integral in Equation 2.48, and the function $1-e^{-b_{t o r}(a) \gamma}$. The aspect ratio for this example is $a=3$, and $b_{\text {tor }}(a)=3.44$.

$b_{\text {tor }}(a)$ that best fit the exact solution for different aspect ratios are shown in Figure 2.15 together with a fit given by

$$
b_{\text {tor }}(a)=1.7143\left(2.1582-e^{-0.60222 a}\right)
$$

With this approximation, the total flux for side-on emission of a torus is

$$
F_{s o}(\nu)=(4 a+\pi) \frac{r^{2}}{d^{2}} S(\nu)\left[1-e^{-b_{t o r}(a) \gamma(\nu)}\right]
$$

An example is shown in Figure 2.16, where the aspect ratio of the torus is $a=3$.

In this geometry the ratio between the face-on/side-on flux for high and low values of $\gamma(\nu)$ is

$$
\frac{F_{f o} / F_{s o}(a, \gamma(\nu) \rightarrow \infty)}{F_{f o} / F_{s o}(a, \gamma(\nu) \rightarrow 0)}=\frac{b_{t o r}(a)}{1.45}
$$

As previously discussed, the higher this ratio, the more important are geometric effects and therefore the more sensitive a measurement of opacity of the material will 
be. It can be seen from Equation 2.52 that this ratio is highest for a torus with a high aspect ratio, that is, one whose three-dimensional features are least important. The maximum value for the ratio in Equation 2.52 is obtained for the limit $a=R / r \rightarrow \infty$, and is $\sim 2.5$, which is greater than the hemispherical case, but far from the value for the planar hemi-ellipsoid or cylinder.

\subsection{Summary and conclusions}

In this chapter the interaction between a radiation field and a plasma has been discussed, with a detailed analysis of the equations that govern the problem. It has been shown how for simple cases the escape factor treatment can lead to much faster computational calculations. The radiative transfer equation has been deduced, with the introduction of the concepts of emissivity and opacity of a plasma, and the solution for a uniform one-dimensional plasma has been obtained, leading to the definition of the optical depth as the length of the plasma in terms of mean-free-paths for radiation of a given frequency.

With these definitions, the enhancement of optically thick radiation in plasmas was introduced as a consequence of the different lengths of the mean chord of the plasma and the LOS through the plasma in the direction of a detector. This effect was shown to depend on the plasma geometry.

The solutions of the radiative transfer equation for several geometries (cylinders, hemi-spherical and hemi-ellipsoidal shells and torii) have been deduced along with useful approximations for computational purposes. It was shown how these solutions were different for the face-on and side-on views, depending on the exact geometry, which could be used as an opacity diagnostic method. The exact expressions for the face-on/side-on flux ratio are summarised below. 
- Cylinder:

$$
\frac{F_{f o}}{F_{s o}}(\nu)=2 \frac{\left[1-e^{-a \gamma(\nu)}\right]}{\left[1-\frac{\pi}{2}\left(L_{-1}(2 \gamma(\nu))-I_{1}(2 \gamma(\nu))\right)\right]}
$$

- Hemispherical shell:

$$
\frac{F_{f o}}{F_{s o}}(\nu)=2 \frac{\left[1-e^{-\gamma(\nu)}(1-\gamma(\nu))-\gamma(\nu)^{2} E_{1}(\gamma(\nu))\right]}{\left[1-e^{-2 \gamma(\nu)}(1-2 \gamma(\nu))-4 \gamma(\nu)^{2} E_{1}(2 \gamma(\nu))\right]}
$$

- Hemi-ellipsoidal shell

$$
\frac{F_{f o}}{F_{\text {so }}}(\nu)=\frac{2}{a} \cdot \frac{G_{\text {ell }}^{\text {fo }}(a, \gamma(\nu))}{G_{\text {ell }}^{s o}(a, \gamma(\nu))}
$$

- Torus

$$
\frac{F_{f o}}{F_{s o}}(\nu)=\frac{4 \pi a}{4 a+\pi} \cdot \frac{\left[1-\frac{\pi}{2}\left(L_{-1}(2 \gamma(\nu))-I_{1}(2 \gamma(\nu))\right)\right]}{G_{t o r}^{s o}(a, \gamma(\nu))}
$$

It has been proved that in the cylindrical, hemi-ellipsoidal and toroidal cases, an opacity measurement would be most sensitive to the flux ratio in the limit of a two-dimensional plasma (planar disk, planar hemi-ellipsoid and two-dimensional ring respectively). In particular, the greatest sensitivity is achieved in the limit of a planar plasma. This is not a surprising result, given that in a planar geometry, the lines of sight for the face-on and side-on fluxes can be made arbitrarily different depending on the ratio between the plasma thickness and radius, whereas in the hemi-ellipsoidal or toroidal cases, these lengths are intrinsically related by the symmetry of the plasma. 


\section{Chapter 3}

\section{Cylindrical plasmas in HED}

\section{conditions}

By the end of Chapter 2 it was shown that the effects of the plasma geometry on its X-ray emission are easiest to study in a cylindrical geometry, given that in this case the two charateristic dimensions of the plasma (its thickness $H$ and its radius $R$ ) are independent of each other. In this chapter, the characteristic X-ray spectra of HED cylindrical plasmas are studied further, focusing on practical measurements, and the model used for the spectroscopic simulations included in this thesis is described. Also, a fully-spectroscopic method for measuring the ion density in HED cylindrical plasmas using its $\mathrm{He}-\alpha$ emission is presented.

The chapter is structured as follows. In Section 3.1, a bibliographic review of the different methods for density calculations in plasmas is given, with focus on the interest of the $\mathrm{He}-\alpha$ complex for these methods. A full description of the lines that make up this complex is also given. In Section 3.2, the radiative transfer model used for the simulations presented in this thesis is described, as well as some of the physical effects that immediately appear in the model as a consequence of the optical depth. Section 3.3 adapts the frequency-resolved equations for the face-on and side- 
on flux of a cylinder (obtained in Chapter 2) to the line-integrated flux for Gaussian lineshapes. The corresponding line ratios are then obtained. Section 3.4 describes the mathematical method to measure the ion density of the plasma from said line ratios. A particular example of the application of this method is presented in Section 3.4 .3 and its robustness with respect to deviations from the perfectly cylindrical geometry is shown in Section 3.4 .4 by studying a plasma frustum. Finally a summary is presented in Section 3.5.

Part of the content of this chapter was published in High Energy Density Physics in 2019 [55].

\subsection{Density calculations in HED plasmas}

Measuring the temperature and density of plasmas in HED experiments is fundamental to understanding the conditions of the plasma and to design better experimental set-ups. Spectroscopy is a commonly used method for temperature [24, 25, 26, 27, 28, 29, 81, 82, 83, 84, 85] and plasma density measurements, with various different methods being applied depending on the conditions involved.

In the high density regime, such as the plasma within an ICF implosion core, one of the most common spectroscopic approaches for measuring the plasma density is the study of Stark broadening of spectral lines [83, 86, 87]. However, for plasmas at lower densities, such as the plasma that fills a hohlraum in indirect drive ICF, the Stark broadening is smaller than thermal broadening associated with the Doppler effect. The line profile is then usually Gaussian or Voigt-like, and since the Lorentzian contribution from the Stark effect must be isolated [88], large uncertainties in the measurement are inevitable.

If diagnosis via Stark broadening is not appropriate, there are some other approaches to characterising the plasma density using its continuum emission [89], flu- 


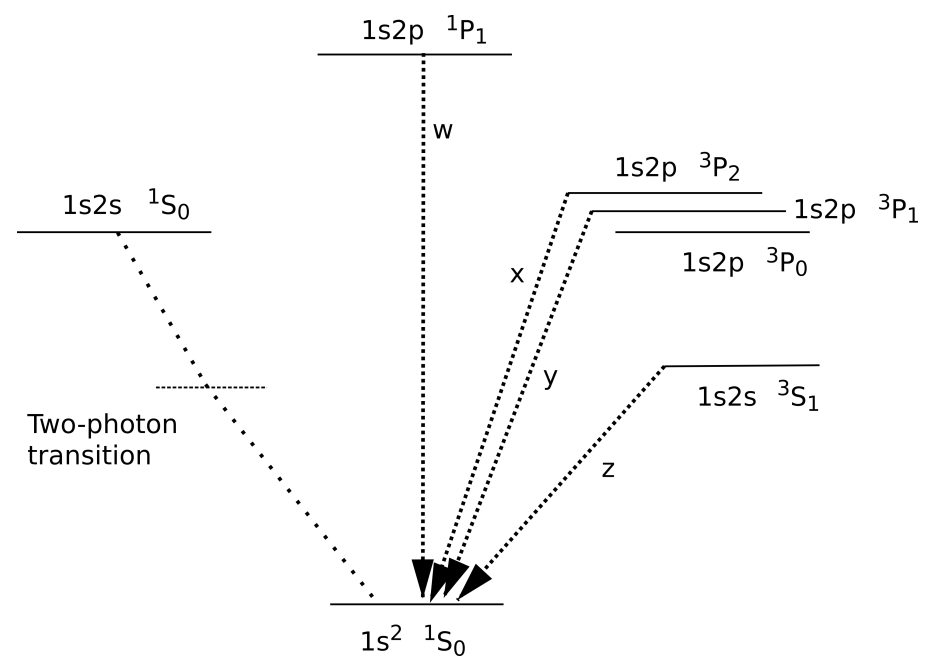

Figure 3.1: Configuration of the excited states of the $\mathrm{He}-\alpha$ complex showing the states and radiative transitions involved.

orescence emission [90], or the study of line ratios, as the relative intensity of lines can provide information about the atomic level population distribution in a plasma and therefore its density.

\subsubsection{Interest of the $\mathrm{He}-\alpha$ complex}

The He- $\alpha$ emission complex $\left(1 s 2 l \rightarrow 1 s^{2}\right)$ is one of the brightest components of the K-shell emission in HED plasmas. This complex is made up of four lines (which are named here according to Gabriel's work [91]), namely the resonance or $w$ line $\left(1 s 2 p{ }^{1} P_{1} \rightarrow 1 s^{2}{ }^{1} S_{0}\right)$, two intercombination lines, the $x$ line $\left(1 s 2 p{ }^{3} P_{2} \rightarrow 1 s^{2}{ }^{1} S_{0}\right)$ and the $y$ line $\left(1 s 2 p^{3} P_{1} \rightarrow 1 s^{2}{ }^{1} S_{0}\right)$; and the forbidden or $z$ line $\left(1 s 2 s{ }^{3} S_{1} \rightarrow 1 s^{2}{ }^{1} S_{0}\right)$. These transitions and the corresponding states are shown in Figure 3.1. Note that there is an additional two-photon transition from the $1 s 2 s{ }^{1} S_{0}$ state, which is not included in the following analysis as the energies of the photons involved are much lower than for the rest of the lines.

While the population of the $1 s 2 p{ }^{1} P_{1}$ state is dictated by direct collisional excitations and radiative emission, the ${ }^{3} P_{2,1,0}$ and ${ }^{3} S_{1}$ states have a longer lifetime and they can interchange population via additional collisional processes [92]. At low densities, 


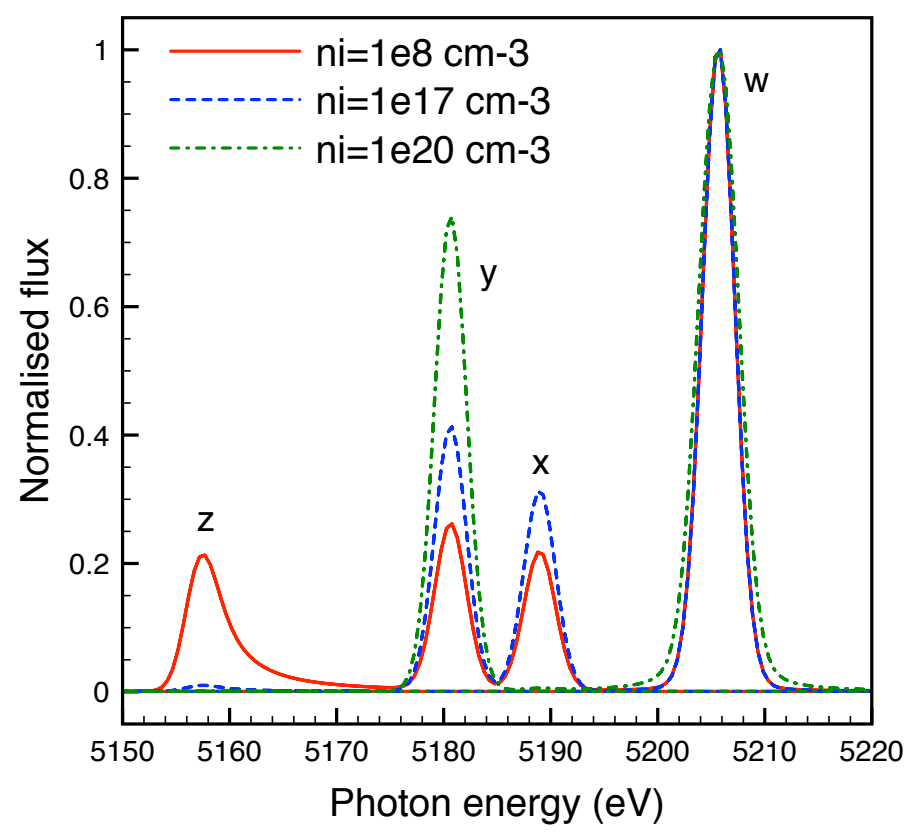

Figure 3.2: Comparison between the emission from He-like V $(\mathrm{Z}=23)$ for different ion densities at a constant electron temperature of $1000 \mathrm{eV}$. Note how the $x$ and $z$ lines are present in a coronal conditions, but they disappear for higher ion densities. The Be-like and Li-like contributions have been removed for clarity.

these collisional processes are in competition with radiative decay, and so emission from all lines can be observed. As the density increases, collisions become more important in balancing the populations, and emission from the $z$ and $x$ line (in this order) diminishes to the point of being unobservable. At the ion densities of interest for this thesis $\left(n_{i} \sim 10^{19}-10^{20} \mathrm{~cm}^{-3}\right)$ the $w$ and $y$ lines are the only remaining He-like components, usually blended with Li-like satellite emission $\left(1 s 2 l n l^{\prime} \rightarrow 1 s^{2} n l^{\prime}\right)$. This is shown in Figure 3.2 , where the simulated flux from the $\mathrm{He}-\alpha$ complex of optically thin vanadium $(\mathrm{Z}=23)$ is shown for different ion densities at a constant electron temperature of $1000 \mathrm{eV}$, and it can be seen how increasing the ion density causes the $z$ line first, and the $x$ line second to disappear from the spectrum. The flux has been normalised to the peak of the $w$ line and the Li-like emission has been removed for ease.

In 1969, Gabriel and Jordan [92] developed a method for characterization of the 
solar corona that uses line ratios from the $\mathrm{He}-\alpha$ complex. In particular, their method uses the ratio of intensities $z /(x+y)$ to characterise the electron density of the plasma. Gabriel and Jordan's method has been widely used for different regions of the solar corona [93, 94, 95, 96] and given its great potential, there have also been many efforts to extend it to different astrophysical plasmas, such as planetary nebulae [97] and stellar winds and coronae [98, 99, 100, 101]. However, this method is only valid at relatively low ion densities $\left(n_{i}<10^{12}-10^{13} \mathrm{~cm}^{-3}\right)$, as for higher densities there is no emission from the $x$ and $z$ lines.

The use of the ratio of the two remaining lines $w / y$ as a density diagnostic for laser-generated plasmas was first studied by Vinogradov et al. [102] and the method was further improved in the following years [103, 104, 105]. After that, the $w / y$ ratio became a commonly used diagnostic for measuring the density of laser-produced plasmas [106, 107, 108, 109] and Tokamaks [110, 111]. Nevertheless, as the opacity of the $w$ line is several times greater than that of the $y$ line, in $\mathrm{HED}$ conditions the $w$ line usually becomes optically thick whereas the $y$ line does not. Therefore, opacity effects and plasma geometry must be taken into account when making diagnostic inferences based upon those lines, as explained in Chapter 2.

\subsection{Cretin model}

As mentioned in Section 1.2, in order to produce accurate and reliable simulations to study the spectral emission of HED cylindrical plasmas, the two-dimensional mode of the radiative transfer code Cretin [31] was used.

The model consists of a cylindrical plasma with arbitrary radius and thickness. The number of computation nodes along the axial and radial direction is selected for each case so that the calculations converge. The plasma is initialised at a given temperature and density, with these conditions being completely uniform with no 


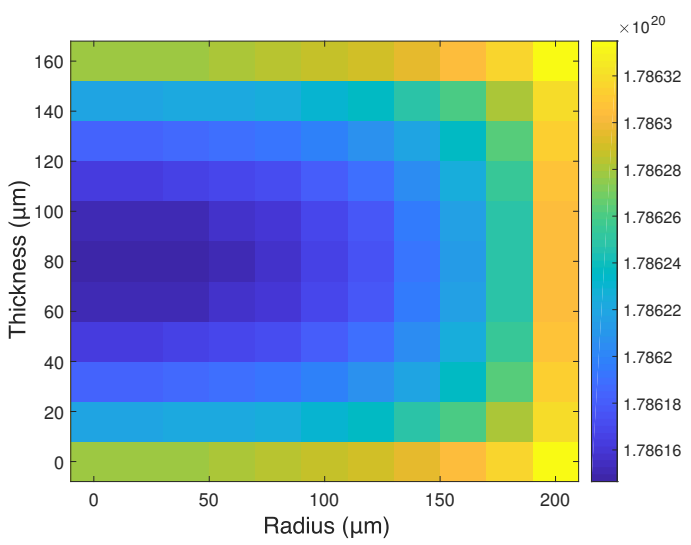

(a)

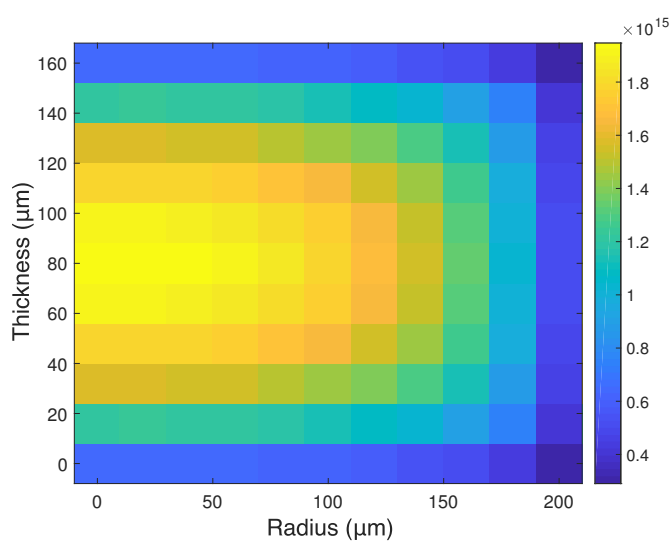

(b)

Figure 3.3: Example of the spatial distribution of the populations of the ground (3.3a) and the excited (3.3b) states of the $w$ line transition in a plasma cylinder, as obtained from the Cretin model.

boundary effects. The plasma cylinder lies on the $z=0$ plane and two spectral detectors are implemented, to measure the face-on and side-on spectral flux. The face-on detector is placed at $(0, H+d)$ - using $(r, z)$ coordinates - and looking in the $-z$ direction, whereas the side-on detector is placed at the coordinates $(R+d, H / 2)$, looking in the $-r$ direction, where $R$ and $H$ are the radius and thickness of the cylinder respectively and $d$ is a positive distance to ensure that the detectors are outside the plasma. Both detectors are designed to observe the whole extent of the plasma.

By letting the plasma evolve for a few iterations of the code so that the radiation field reaches equilibrium with the plasma (usually two iterations are enough with this model), a physically realistic distribution of the population of the atomic states can be obtained, showing the effect of the radiation field. An example of these distributions is shown in Figure 3.3. These figures correspond to the ion populations of the ground (3.3a and excited (3.3b states of the $w$ line of the $\mathrm{He}-\alpha$ complex in a $1500 \mathrm{eV}$ titanium $(\mathrm{Z}=22)$ plasma with an ion density $n_{i}=2.0 \times 10^{19} \mathrm{~cm}^{-3}$. The plasma radius is $200 \mu \mathrm{m}$ and its thickness $160 \mu \mathrm{m}$.

It can be seen how in the centre of the cylinder, the ground state is depopulated 


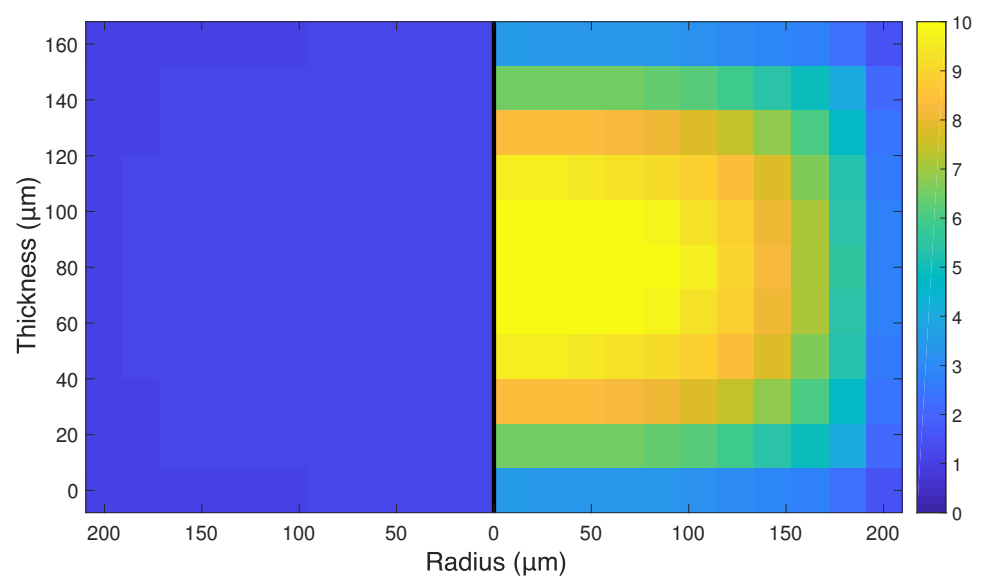

Figure 3.4: Increase in the population of the excited states of the $y$ (left) and $w$ (right) transitions across a cylindrical plasma due to radiation transport effects, as obtained from the Cretin model.

in favour of the excited state. This is caused by photons from the line emission being absorbed within the plasma volume and photopumping the transition, as described in Section 2.2. Given that in the plasma boundaries there are fewer photons colliding with the ions, as photons that escape the plasma cannot come back in, the photopumping effect is lower, thus creating this characteristic distribution.

As a result of Equation 2.6, the radiation field will modify the population of all atomic states, to a greater or lesser extent, depending on the optical depth. To illustrate this, Figure 3.4 shows the factor by which the population of the excited states of the $y$ (left) and $w$ (right) lines, whose optical depths differ by a factor of 22 for titanium, increase when radiation transport is included in the model with respect to the case with no radiation transport. It can be seen how the population of the excited state of the $y$ line barely changes across the cylinder, whereas for the $w$ line, the population at the centre of the cylinder (where the radiation field is more intense) increases by a factor of $\sim 10$.

This increase in the population of the excited state of the $w$ line is an indicator of the potential enhancement of optically thick lines due to optical depth effects, as explained in Section 2.2. Measuring and characterising this effect in real HED 
experiments is the motivation of this thesis and the aim of the experiments described in Chapter 4.

\subsection{Line ratios in cylindrical geometry}

In Section 3.1 it was discussed how the ratio of the flux from the $w$ and $y$ lines could be used as a density diagnostic in HED conditions. Also, Chapter 2 , shows how the ratio between the spectral flux in different directions for non-spherical geometries can provide information about the plasma opacity. Therefore, a combination of these two ratios can potentially be used as a more complete diagnostic. However, in most experimental applications it is not possible to accurately measure the frequency-resolved line ratios, due to the presence of noise in the data, or instrumental broadening effects. For this reason, it is common to work with the line-integrated ratios, which account for the emission across the whole width of the line. These ratios are calculated in this section.

The plasma of study is assumed to be perfectly cylindrical and completely uniform (single temperature and density). Time variations will be neglected, as explained in Section 2.1.2. The temperatures of study are of the order of $1-2 \mathrm{keV}$ and the ion densities have values of $10^{18}-10^{20} \mathrm{~cm}^{-3}$. Under this conditions, Doppler broadening is approximately one order of magnitude greater than Stark broadening 11] [113, 114, 115] and therefore Gaussian lineshapes are assumed, although the calculations can be extended to different lineshapes, such as a Lorentzian or a Voigt profile.

For a single Gaussian-shaped line, the emissivity and opacity coefficients can be written as

$$
\begin{aligned}
& \eta(x)=\eta_{0} e^{-x^{2}} \\
& \kappa(x)=\kappa_{0} e^{-x^{2}},
\end{aligned}
$$

\footnotetext{
${ }^{1}$ This result was verified using the atomic kinetics code SCRAM [112 and Cretin.
} 
where $\eta_{0}$ and $\kappa_{0}$ are respectively the emissivity and opacity at line centre, and $x$ is the dimensionless frequency

$$
x=\frac{\nu-\nu_{0}}{\nu_{D}}
$$

with $\nu_{0}$ the frequency of the transition at line centre and $\nu_{D}$ the Doppler width. Therefore, using Equation 2.16, it is possible to write the intensity of a particular line as

$$
I(x, z)=\frac{\eta(x)}{\kappa(x)}\left(1-e^{-\kappa(x) z}\right)=S_{0}\left(1-e^{-\kappa(x) z}\right)
$$

where $S_{0}$ is the source function at line centre.

Combining this expression with the face-on flux of a plasma cylinder from Equation 2.23 , the integrated line flux emitted by a plasma cylinder in the axial direction can be obtained as

$$
F_{\text {line }}^{\text {face }}=\frac{\pi R^{2}}{d^{2}} S_{0} \int_{-\infty}^{+\infty} d x\left(1-\exp \left[-\kappa_{0} H e^{-x^{2}}\right]\right)
$$

Following the same procedure and using Equation 2.27, the integrated line flux for side-on emission is

$$
F_{\text {line }}^{\text {side }}=\frac{2 H R}{d^{2}} S_{0} \int_{-\infty}^{+\infty} d x\left(1-\exp \left[-1.45 \kappa_{0} R e^{-x^{2}}\right]\right)
$$

Using a similar notation to that in Chapter 2, the integrals in Equations 3.4 and 3.5 are hereafter written as $G^{\text {face }}$ and $G^{\text {side }}$ respectively. The flux corresponding to a particular line $(w$ or $y$ ) is specified by denoting $w$ or $y$ as a subscript, considering only the line-integrated fluxes, unless otherwise specified. The subscript 0 for the source function is also dropped, always considering its value at line centre.

With this notation it is now possible to write the line ratio $w / y$ for either face-on or side-on view as

$$
\left.\frac{F_{w}}{F_{y}}\right|_{\text {view }}=\frac{S_{w} G_{w}^{\text {view }}}{S_{y} G_{y}^{\text {view }}}
$$


Given that the source functions of both lines are independent of the direction of emission for uniform plasmas (they are a function of the temperature and density of the plasma), if the $w / y$ ratios for both views are divided, the result depends only on the $G$ integrals for both lines in both views.

$$
\frac{F_{w} /\left.F_{y}\right|_{\text {face }}}{F_{w} /\left.F_{y}\right|_{\text {side }}}=\frac{G_{w}^{\text {face }}}{G_{w}^{\text {side }}} \cdot \frac{G_{y}^{\text {side }}}{G_{y}^{\text {face }}} .
$$

The equations deduced here are used in the next section to calculate the ion density of the plasma. In the conditions of interest, it is required that the $w$ line is significantly optically thick $\left(1-e^{-\tau_{w}} \sim 1\right.$ near line centre) whereas the $y$ line is not. However, for the method presented here, it is not necessary that the $y$ line is strictly optically thin $\left(\tau_{y}<1\right)$, but it can have optical depth of a few. For the remainder of this chapter, it will be assumed that these conditions hold.

\subsection{A fully spectroscopic method for measuring the ion density}

In this section, a method for measuring the ion density of a cylindrical plasma using only its spectra is presented. This method requires two measurements of the $\mathrm{He}-\alpha$ spectrum (the spectral flux in the axial and the radial directions), one of which can be the already existent measurement in the Dot Spectroscopy platform for temperature diagnostic, described in detail in Chapter 5. A single element plasma is considered for simplicity. This is not a necessary assumption, as the method is still valid for a multi-species plasma as long as no lines from one element overlap with the $\mathrm{He}-\alpha$ complex of another. In the case of more than one element being present in the plasma, the $\mathrm{He}-\alpha$ spectrum of each element can be used to obtain the ion density of that particular element. The total ion density can then be calculated as the sum of the 
individual contributions, provided that the assumption of a uniform plasma holds.

\subsubsection{Aspect ratio}

In most experiments, it is not possible to obtain direct measurements of the size of the plasma and the spectral flux in both directions at the same time. However, the fact that the $w$ line is optically thick makes it possible to obtain information about the plasma geometry just from the ratio of the spectral fluxes in two directions, without explicitly measuring the plasma size. In particular, the aspect ratio $\beta=H / R$ can be calculated. If the $w$ line fluxes for both views are divided, then

$$
\frac{\left.F_{w}\right|_{\text {face }}}{\left.F_{w}\right|_{\text {side }}}=\frac{\pi R}{2 H} \frac{G_{w}^{\text {face }}}{G_{w}^{\text {side }}} .
$$

In the limit of high values of the optical depth, the ratio of the $G$ functions can be simplified. Using L'Hôpital's rule once

$$
\frac{G_{w}^{\text {face }}}{G_{w}^{\text {side }}} \sim \lim _{\kappa_{0} \rightarrow \infty} \frac{H}{1.45 R} \frac{\int_{-\infty}^{+\infty} d x \exp \left[-\kappa_{0} H e^{-x^{2}}\right] e^{-x^{2}}}{\int_{-\infty}^{+\infty} d x \exp \left[-1.45 \kappa_{0} R e^{-x^{2}}\right] e^{-x^{2}}}
$$

and then Holstein's approximation for the resulting integrals [59], one obtains

$$
\frac{G_{w}^{\text {face }}}{G_{w}^{\text {side }}} \sim \sqrt{\frac{\ln \left(1.45 \kappa_{w} R\right)}{\ln \left(\beta \kappa_{w} R\right)}}=\sqrt{\frac{\ln \left(1.45 \cdot A \tau_{0}\right)}{\ln \left(A \beta \tau_{0}\right)}}
$$

where $\tau_{0}=\kappa_{y} R$. The factor $A=\kappa_{w} / \kappa_{y}$ is the ratio of the opacity of the $w$ and the $y$ lines, given by the ratio of the oscillator strengths of the transitions and therefore fixed for each element. It can be adjusted for the element of consideration, and it is independent of temperature and density. This factor is introduced here as it is easier to work with the optical depth of the $y$ line, than the $w$ line. The dependence of the ratio $G_{w}^{f a c e} / G_{w}^{s i d e}$ with the optical depth of the $y$ line for different aspect ratios, for $A=15$ is shown in Figure 3.5 


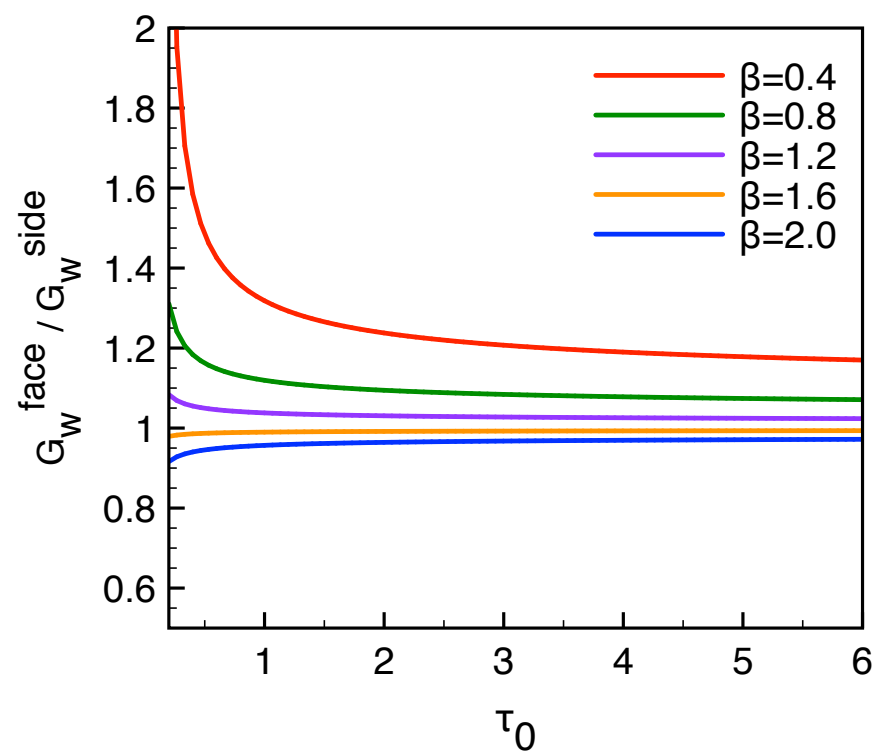

Figure 3.5: $G_{w}^{\text {face }} / G_{w}^{\text {side }}$ ratio as a function of the optical depth of the $y$ line for a variety of aspect ratios, when $\kappa_{w} / \kappa_{y}=15$. For the values considered in this work ( $\tau_{0}$ of the order of a few), this value is not strongly dependent on $\tau_{0}$, except for low aspect ratios

From Equations 3.8 and 3.10 , it is now possible to obtain the following expression for the aspect ratio $\beta=H / R$

$$
\beta=\frac{\pi}{2} \cdot \frac{\left.F_{w}\right|_{\text {side }}}{\left.F_{w}\right|_{\text {face }}} \sqrt{\frac{\ln \left(1.45 \cdot A \tau_{0}\right)}{\ln \left(A \beta \tau_{0}\right)}}
$$

And therefore, Equation 3.7 can be now written explicitly as

$$
\frac{F_{w} /\left.F_{y}\right|_{\text {face }}}{F_{w} /\left.F_{y}\right|_{\text {side }}}=\sqrt{\frac{\ln \left(1.45 \cdot A \tau_{0}\right)}{\ln \left(A \beta \tau_{0}\right)}} \cdot \frac{\int_{-\infty}^{+\infty} d x\left(1-\exp \left[-1.45 \tau_{0} e^{-x^{2}}\right]\right)}{\int_{-\infty}^{+\infty} d x\left(1-\exp \left[-\tau_{0} e^{-x^{2}} \beta\right]\right)},
$$

where the notation $\tau_{0}=\kappa_{y} R$ is used to indicate that, although $\kappa_{y}$ and $R$ cannot be calculated separately, their product is an independent variable in Equations 3.11 and 3.12. Physically, $\tau_{0}$ corresponds to the optical depth of a $y$ line photon (at line centre) travelling a distance equivalent to the radius of the cylinder, $R$. Equivalently, it can be understood as the radius of the cylinder in units of the mfp of a $y$ line-centre photon. 
Equations 3.11 and 3.12 can now be solved together thus obtaining $\beta$ and $\tau_{0}$. From direct manipulation of Equation 3.11, it is possible to obtain a direct definition of $\tau_{0}$ as a function of $\beta$ as

$$
\tau_{0}=\frac{1}{A} \exp \left[\frac{\beta^{2} \ln (\beta)-C^{2} \ln (1.45)}{C^{2}-\beta^{2}}\right]
$$

where

$$
C=\frac{\pi}{2} \cdot \frac{\left.F_{w}\right|_{\text {side }}}{\left.F_{w}\right|_{\text {face }}}
$$

which can be directly measured from the spectra. The function in Equation 3.13 is monotonically decreasing with $\beta$ for a given value of $C$, which implies that for a given aspect ratio, the corresponding value of $\tau_{0}$ is unique. Note that when $\beta \sim 1.45$, from Equation 3.11 it follows that $\beta \sim C$, which corresponds to a pole in the function in Equation 3.13 . This is because when the aspect ratio approaches 1.45 (or values close to $\pi / 2$ ), the face-on and side-on flux become very similar (this can be easily seen by comparing Equations 3.4 and 3.5 and independent of the optical depth. Therefore, for these geometries, the line ratios between different views provide no information whatsoever about the plasma.

It is now possible to write $\tau_{0}=f_{1}(\beta, C)$, and substitute this expression into Equation 3.12 , which can now be written as

$$
\frac{F_{w} /\left.F_{y}\right|_{\text {face }}}{F_{w} /\left.F_{y}\right|_{\text {side }}}=\sqrt{\frac{\ln \left(1.45 \cdot A f_{1}(\beta, C)\right)}{\ln \left(A \beta f_{1}(\beta, C)\right)}} \cdot \frac{\int_{-\infty}^{+\infty} d x\left(1-\exp \left[-1.45 f_{1}(\beta, C) e^{-x^{2}}\right]\right)}{\int_{-\infty}^{+\infty} d x\left(1-\exp \left[-f_{1}(\beta, C) e^{-x^{2}} \beta\right]\right)},
$$

and is just a function of the aspect ratio $\beta$ and the observable $C$. Defining $f_{2}(\beta, C)=$ $\frac{F_{w} /\left.F_{y}\right|_{\text {face }}}{F_{w} /\left.F_{y}\right|_{\text {side }}}$ for simplicity, it is possible to study the uniqueness of $\beta$ for a given pair of values of $C$ and $f_{2}(\beta, C)$. Figure 3.6 shows the value of $f_{2}(\beta, C)$, and it can be seen how the function decreases monotonically with $C$ and increases monotonically with $\beta$. This implies, that for a given value of $C$ and $f_{2}(\beta, C)$, which are both measured 


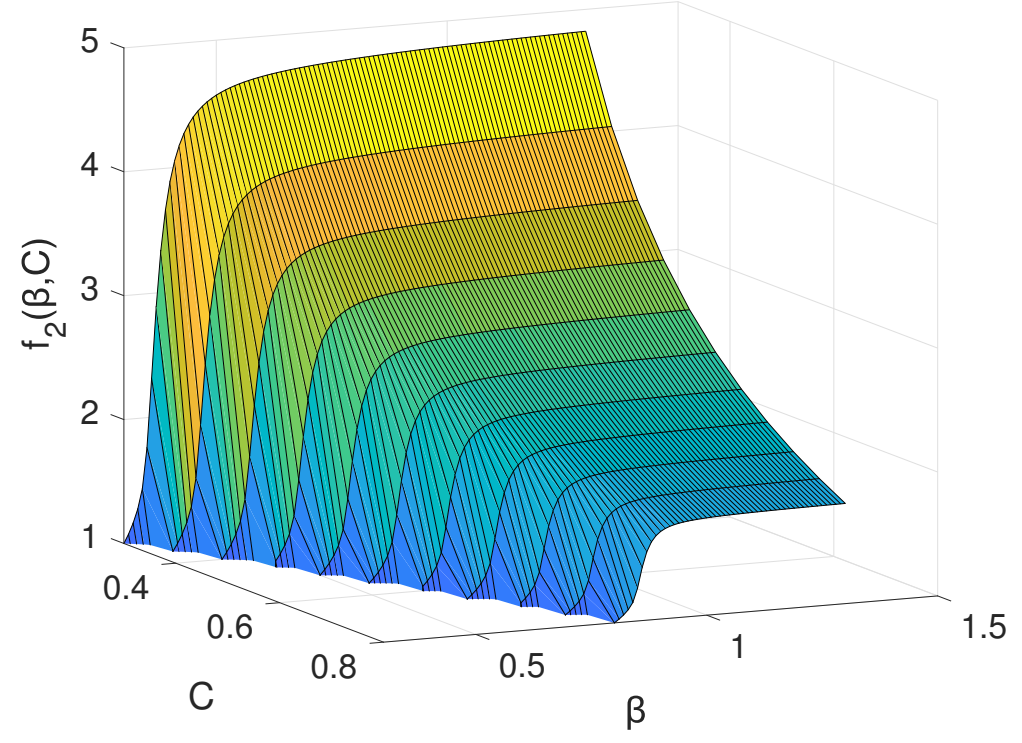

Figure 3.6: Map of the values of the observable $f_{2}(\beta, C)=\frac{F_{w} /\left.F_{y}\right|_{\text {face }}}{F_{w} /\left.F_{y}\right|_{\text {side }}}$, given by Equation 3.15, as a function of the aspect ratio of the cylinder $\beta=H / R$, and the observable $C$, given in Equation 3.14 .

from the face-on and side-on spectra, there is only one value of the aspect ratio $\beta$ that satisfies Equation 3.12. Furthermore, as it was explained above, for a given value of $\beta$, there is only one possible value of $\tau_{0}$ that satisifes Equation 3.11, which is given by Equation 3.13 .

This concludes that for every pair of values $C$ and $f_{2}$ - which are values directly extracted from the experiment and therefore, known - there is only one pair of values $\beta$ and $\tau_{0}$ that satisfy Equations 3.11 and 3.12 at the same time. The solution can be obtained by solving Equation 3.15 numerically for $\beta$ and then calculating $\tau_{0}$ from Equation 3.13 .

\subsubsection{Calculation of the ion density}

The optical depth parameter $\tau_{0}$ is related to the ion density of the plasma by

$$
\tau_{0}=n_{i} F \sigma_{0} R,
$$


where $n_{i}$ is the number density of ions, $F$ is the fraction of ions in the ground state of the transition (which, for the case of the $y$ line is the He-like ground state $1 s^{2}{ }^{1} S_{0}$ ) and $\sigma_{0}$ is the cross section for photon absorption at line centre. For a line profile $\phi(x)$, the cross-section is

$$
\sigma_{0}=\frac{\pi e^{2}}{m_{e} c} f \phi(0)
$$

where $f$ is the oscillator strength of the transition, in this case the $y$ line. The ion density $n_{i}$ can be related to the total number of atoms in the plasma, $N$ and its volume $V$ as

$$
n_{i}=\frac{N}{V}=\frac{N}{\pi \beta R^{3}}
$$

The quantity $N$, in turn, can be expressed as the initial mass of the target divided by the atomic mass, $N=M / m_{a}$ so that

$$
n_{i}=\frac{M}{m_{a} \pi \beta R^{3}}
$$

Combining Equations 3.16 and 3.19 , the following expression for the ion density is obtained

$$
n_{i}=\left(\frac{m_{a} \pi \beta}{M}\right)^{1 / 2}\left(\frac{\tau_{0}}{F \sigma_{0}}\right)^{3 / 2}
$$

Equation 3.20 allows one to calculate the ion density of a plasma from its aspect ratio $\beta$ and the optical depth of the $y$ line at line centre $\tau_{0}$. As shown in Equations 3.11 and 3.12 , these two parameters can be calculated from the ratio of fluxes between the $w$ and the $y$ line for face-on and side-on views.

\subsubsection{A particular example}

This method was tested using the model described in Section 3.2. The cylinder consists of a titanium plasma, with a radius $R=200 \mu \mathrm{m}$ and an aspect ratio $\beta=0.8$, thus fixing the height to $H=160 \mu \mathrm{m}$. The temperature of the plasma is $1500 \mathrm{eV}$ and 


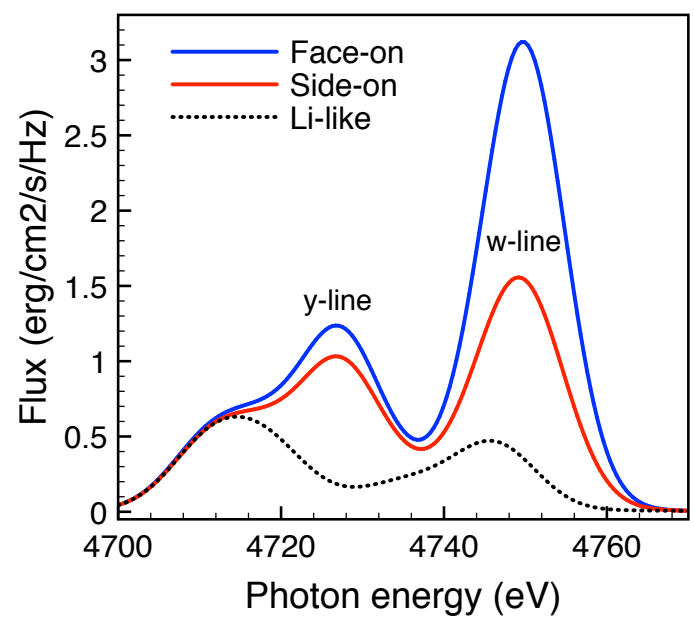

(a)

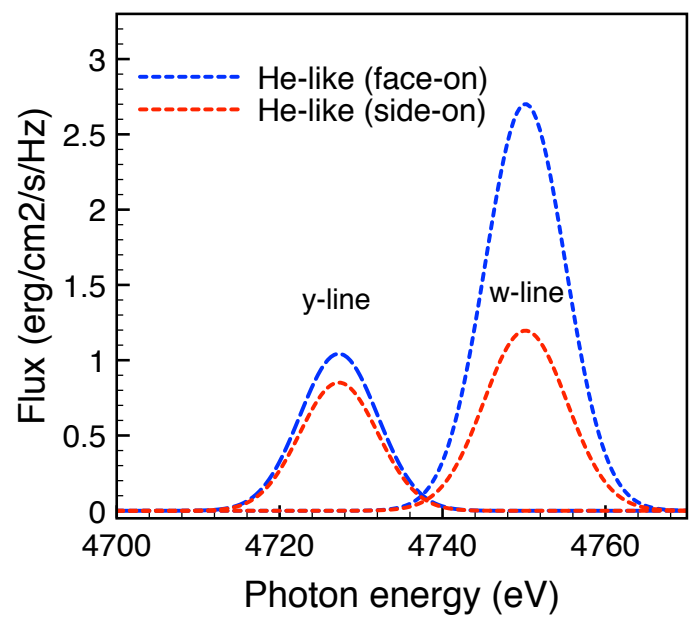

(b)

Figure 3.7: Spectra of the He-like complex for the example case. These correspond to the face-on and side-on flux of a Ti cylinder with an aspect ratio $\beta=0.8$ an ion density of $n_{i}=2 \times 10^{19} \mathrm{~cm}^{-3}$ at $T=1500 \mathrm{eV}$. Figure $3.7 \mathrm{a}$ shows the total flux including He-like and Li-like satellites. The Li-like contribution for both cases is shown as the black dotted line. In Figure 3.7b, the satellite contribution has been subtracted and the He-like contribution is shown. The only remaining components are the $w$ and $y$ lines, that can now be fitted to two Gaussians to apply the process described in this chapter.

the plasma is in steady state. The ion density is fixed to a value of $n_{i}=2.0 \times 10^{19} \mathrm{~cm}^{-3}$. These conditions correspond to those shown in Figure 3.3. The total number of ions is then $N=V n_{i}=4.02 \times 10^{14}$. The side-on and face-on spectra obtained from the simulation are shown in Figure 3.7a, where the positions of the $w$ and $y$ lines are indicated.

As the temperature of the cylinder is known in the simulation (in practice it can be determined self-consistently from spectroscopic measurements), the Li-like structure can be modeled and subtracted from the data, because the Li-like satellite flux is optically thin and therefore, its spectral distribution is density-independent, and its magnitude is independent of the viewing orientation in the conditions of interest (see Equation 2.21. The Li-like structure is shown as the black dotted line in Figure $3.7 \mathrm{a}$. The He-like flux is then obtained for each view, from which the $w$ and $y$ lines can be 
extracted. The He-like lines are shown in Figure $3.7 \mathrm{~b}$.

In this particular example, the flux ratios obtained are:

$$
\begin{aligned}
& \frac{\left.F_{w}\right|_{\text {face }}}{\left.F_{w}\right|_{\text {side }}}=2.21, \\
& \frac{\left.F_{w}\right|_{\text {face }}}{\left.F_{y}\right|_{\text {face }}}=2.68, \\
& \frac{\left.F_{w}\right|_{\text {side }}}{\left.F_{y}\right|_{\text {side }}}=1.50 .
\end{aligned}
$$

The value of $\beta$ and $\tau_{0}$ can now be obtained as described above. For this example, an iterative method was used instead, in order to explore the uncertainties obtained in the ion density. This iterative method consists of introducing a first numerical estimation of $\beta$ and $\tau_{0}$ in the square root in Equation 3.11, thus obtaining a new value of $\beta$ on the left hand side of that same equation. This newly obtained value for $\beta$ is introduced then in Equation 3.12, which is now numerically solved for $\tau_{0}$. The newly calculated values of $\beta$ and $\tau_{0}$ become the new estimated values and the process is repeated until consistency is achieved and the initial estimations are equal to the values obtained after iterating.

From the plasma temperature $(1500 \mathrm{eV})$, the cross section for photon scattering at the centre of the $y$ line is calculated, obtaining $\sigma_{0}=1.70 \times 10^{-18} \mathrm{~cm}^{2}$. The fraction $F$, of ions in the He-like ground, that is, the product of the He-like ionic fraction and the fractional ground level population, can be estimated from the known temperature in the calculations. Its value is 0.89 at this temperature, and therefore, the ion density calculated from Equation 3.20 is

$$
n_{i}=2.0 \times 10^{19} \mathrm{~cm}^{-3}
$$


In a real experiment, there will be experimental errors in the flux of the $w$ and $y$ lines in both directions, which will affect the measurement of the $w / y$ ratio. In addition, as it can be seen in Figure 3.7a, the apparent flux of the He lines has an important contribution from the Li-like satellites that needs to be removed with a model, introducing the potential for additional errors in the line flux. These sources of error will cause the measured $w / y$ ratios to differ from the theoretical ones, and will lead to an error on the calculated ion density. To study the sensitivity of this method to these errors, the flux from each line in both views was allowed to vary randomly within a $10 \%$ of the value from the simulations. This relatively standard value for the error is estimated assuming that the temperature of the plasma is known from other spectroscopic measurements, and that the plasma is uniform. In case the temperature is not known, or there are non-uniformities in the plasma, the shape of the Li-like satellites will have a greater uncertainty and therefore, the error will be greater and there would be additional problems, as the cross-section for photon absorption also depends on the temperature. With this error included in the calculation, the ion density obtained is

$$
n_{i}=2.0 \pm 0.4 \times 10^{19} \mathrm{~cm}^{-3}
$$

As the simulation had a fixed ion density value of $n_{i}=2.0 \times 10^{19} \mathrm{~cm}^{-3}$, there is good agreement with the calculated result.

\subsubsection{Deviations from cylindrical geometry}

During this chapter, it has been assumed that the plasma is a perfect cylinder. However, in practical cases, that is not a realistic assumption, as hydrodynamic forces will act upon the plasma, and the geometry is likely to be deformed. Hydrodynamic effects can be mitigated using a low-Z tamper that provides some confinement pressure and keeps to plasma from expanding [56, 178, 116]. Nevertheless, as much as the 


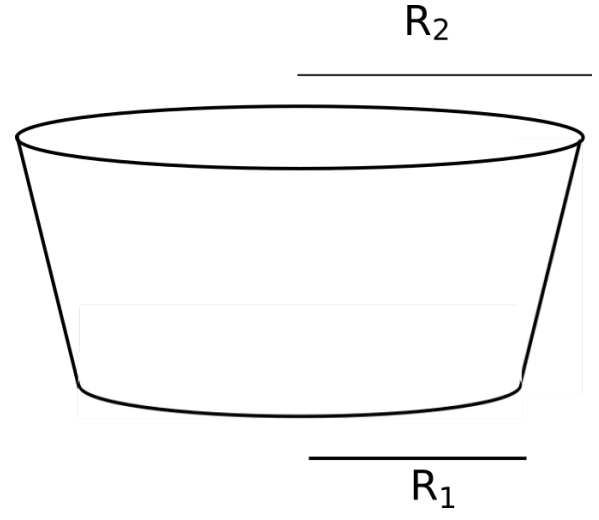

(a)

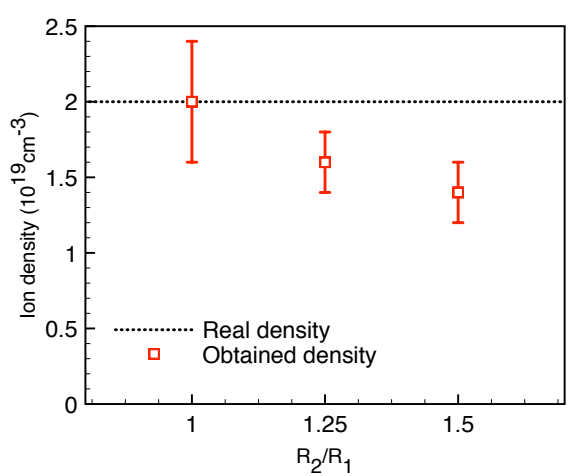

(b)

Figure 3.8: 3.8a Schematic drawing of the frustum geometry. 3.8b Ion density obtained applying this method to Cretin simulations for a frustum as a function of the ratio between the radii of its bases. The point with $R_{2} / R_{1}=1$ corresponds to the cylindrical case shown in Section 3.4.3.

plasma can be confined, a perfect cylindrical shape is never achieved in experiments.

To study how this density characterisation method behaves when the plasma geometry differs from a perfect cylinder it was considered that the plasma expands in solid angle into a frustum (Figure 3.8a), therefore losing its cylindrical geometry. This case can also be studied with the Cretin model defined in the previous section allowing the radius of the cylinder to depend on the $z$ coordinate. For this purpose, every parameter in the simulation was kept identical, except the radius $R_{2}$ of the frustum, defined in Figure 3.8a.

As the cylinder expands in solid angle, the results obtained for the ion density start differing from the real value. The difference increases with the ratio between the two radii of the cylinder. Using the results directly extracted from the simulations, for values of this ratio up to 1.50 in this particular example, the density obtained with this method is in agreement with the correct value within a factor of 2 , as Figure $3.8 \mathrm{~b}$ shows.

These results show that, even if the cylinders expand into solid angle, these method can still be used for obtaining the ion densities with good agreement within the expected experimental errors. 


\subsection{Summary and conclusions}

In this chapter, the interest of the He- $\alpha$ complex for diagnosing the plasma density in HED experiments has been described. The spectral flux from this complex has been analysed in terms of the $w$ and $y$ lines. Since in most experiments it is not possible to frequency-resolve the spectral emission from individual lines, focus was put on the line-integrated flux under each line and their ratios. In particular, the line-integrated flux ratios were obtained for the face-on and side-on emission of a completely uniform and perfectly cylindrical plasma.

The computational model used for the cylindrical simulations in this thesis was also described, showing how the enhancement of optically thick lines with respect to their optically thin limit appears in said simulations as an immediate consequence of the interaction between the plasma and the radiation field. It was shown how photons from an optically thick line get absorbed within the plasma, thus photopumping the excited state of the transition, whereas for optically thin lines this effect does not happen.

In addition, a novel method to obtain the ion density in a cylindrical HED plasma has been introduced. This method only requires spectroscopic measurements of the $\mathrm{He}-\alpha$ region of the spectrum and can be complemented with different spectroscopic measurements of the plasma temperature. It is a general method for HED cylindrical plasmas. This method makes use of the difference in optical depth of the $w$ and $y$ lines to use their ratio from face-on to side-on view as a measurement of the plasma geometry and its optical depth. It was shown that, although a perfectly cylindrical plasma was assumed, small deviations from a cylindrical geometry into a frustum do not critically affect the outcome from this method, and that experimental errors up to a $10 \%$ are acceptable, and the right ion density is obtained within an order of magnitude. 


\section{Chapter 4}

\section{Experimental application: The 'OpticalDepth' campaign}

In this chapter the first experiments of the so-called 'OpticalDepth' campaign at OMEGA are described. These experiments study the angular distribution of the X-ray emission in HED cylindrical plasmas. The computational model described in Chapter 3 as well as some of the results obtained for the line ratios in different views of a cylinder are applied here to study the experimental results.

The chapter is structured as follows. In Section 4.1 the different diagnostic instruments that were fielded in the experiment are described. Section 4.2 describes the targets and the laser configuration, giving a general overview of the experimental set-up. The results are given in Section 4.3. In particular, a comparison between the ion density obtained by directly measuring the plasma size, and using the method described in Chapter 3 is shown. At the end of this section, the measured line ratios for the face-on and side-on view of the generated plasma cylinder are compared with the results from the Cretin model for both a cylinder and a spherical plasma, showing the differences between both cases. Using the results from Chapter 3 , Section 4.4 shows how the experimental data from these experiments can be used to extract 
some information about the opacity of the target, thus allowing one to compare and discriminate between the atomic models used in different codes. Section 4.5 is dedicated to analysing how the experimental results change when using a different pulse shape, to determine whether a more efficient laser drive could be achieved in future experiments. Finally, a summary is presented in Section 4.6 .

Part of the content of this chapter was published in Physics of Plasmas in 2019 [56]. Note that some of the plots presented here are different from those in the publication. The reason for this difference is that, after the paper was published, new and more powerful data analysis techniques using machine learning (such as genetic algorithms [117] or the maleo optimisation algorithm [118]) became available, which slightly modified the results shown in the paper. Also, more precise atomic models for Cretin were developed, yielding a better spectral analysis.

\subsection{Diagnostic instruments}

This section is dedicated to introducing the main set of diagnostics used, their operating principle and their particular application for the OpticalDepth experiments.

\subsubsection{DANTE}

Dante is an absolutely calibrated diode-array spectrometer installed at the OMEGA laser facility and at NIF. It has 18 X-ray Diode (XRD) channels obtaining the timeresolved absolute flux emitted by the target, plus one Photoconductive Detector (PCD) channel measuring the integrated spectrum, although due to limitations in the LOS dedicated to this diagnostic, only 15 channels can be run simultaneously. In this experiment, there were 14 active channels, covering photon energies from 50 to 20,000 eV. These are specified in Table 4.1 together with the corresponding energy ranges. 
For each channel, radiation escapes the target chamber through a pinhole aperture, goes through the X-ray filters that determine the energy range of said channel and reaches an $\mathrm{XRD}$ with a time resolution of $\Delta t \sim 100 \mathrm{ps}$. The energy range of the high energy channels $(E>600 \mathrm{eV})$ is determined by a combination of $\mathrm{K}$ and L-edge filters and the response of the cathode material of the corresponding $\mathrm{XRD}$. In the low energy channels, in order to mitigate the high photon energy response of the edge filters, grazing angle X-ray mirrors are placed between the X-ray filters and the XRD. The combined response of filters, mirror and XRD produces a narrow spectral range with a well defined cutoff at the edge energy for the low energy channels, whereas for the higher energies the response rises at energies $>5 \mathrm{keV}$. The signal from each channel is timed with an electrical fiducial pulse that is referenced to the timing of the laser pulse, obtaining a sinchronisation better than 200 ps [119, 120, 121, 122].

The absolute combined response function of each channel, as well as its uncertainty are known and characterised. This allows for absolute measurements of the X-ray emission which are commonly used by experimentalists to calculate the radiation temperature in ICF related experiments [123, 124]. Recently, it was shown that $a$ priori information can be introduced in this analysis to elliminate the assumption of a Planckian radiation field, which produces more accurate results [125].

For this particular experiment, Dante was used for direct comparison of the X-ray emission in different energy ranges between different shots.

Table 4.1: Photon energy range for all active DANTE channels.

\begin{tabular}{c|ccccccc}
\hline \hline Ch. No. & 1 & 2 & 3 & 4 & 5 & 6 & 7 \\
Energy (eV) & $50-70$ & $150-180$ & $250-280$ & $450-520$ & $750-820$ & $900-980$ & $980-1050$ \\
\hline Ch. No. & 8 & 9 & 10 & 11 & 12 & 14 & 18 \\
Energy (eV) & $1200-1300$ & $1300-1500$ & $1600-1800$ & $2000-3000$ & $1800-2500$ & $3170-4800$ & $10000-20000$ \\
\hline \hline
\end{tabular}




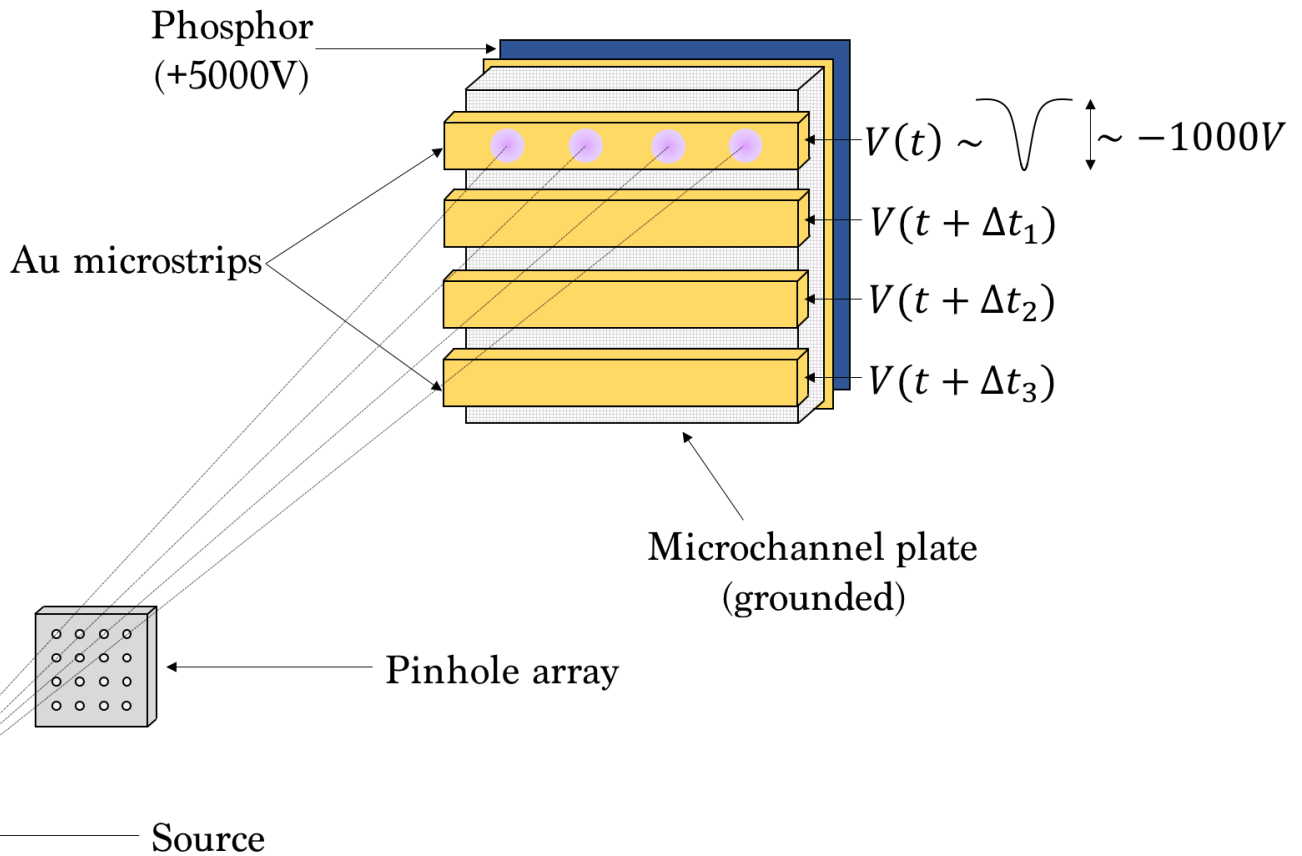

Figure 4.1: Schematic of the XRPINH design used for these experiments. Each of the pinholes creates a different image on the MCP. As each strip of the MCP is activated at a different time, the user can map different moments of the experiment by modifying the delay $\Delta t$. The delay between the different images within a single strip is determined by the time that the voltage pulse takes to travel across the strip.

\subsubsection{Framing cameras}

Framing cameras are X-ray detectors in which different regions are activated at different times, allowing for time-resolved measurements. These measurements are not continuously resolved, but rather grouped in different temporal windows, or 'frames'. The operating principle of framing cameras is illustrated in Figure 4.1. In particular, this figure shows a X-Ray Pinhole Camera (XRPINH), but the principle can be easily extrapolated to different uses.

The core of the camera, shown on the top of Figure 4.1 contains a Microchannel Plate (MCP) whose front face is coated with several $\mathrm{Au}: \mathrm{Cu}$ microstrips whereas the bottom face is completely coated. The MCPs used for this experiment are $0.5 \mathrm{~mm}$ thick glass plates with leaded pores. The pores are $\sim 1 \mu \mathrm{m}$ in diameter and $\sim 10 \mu \mathrm{m}$ apart from each other, covering $50-80 \%$ of the surface of the plates, making the MCPs 


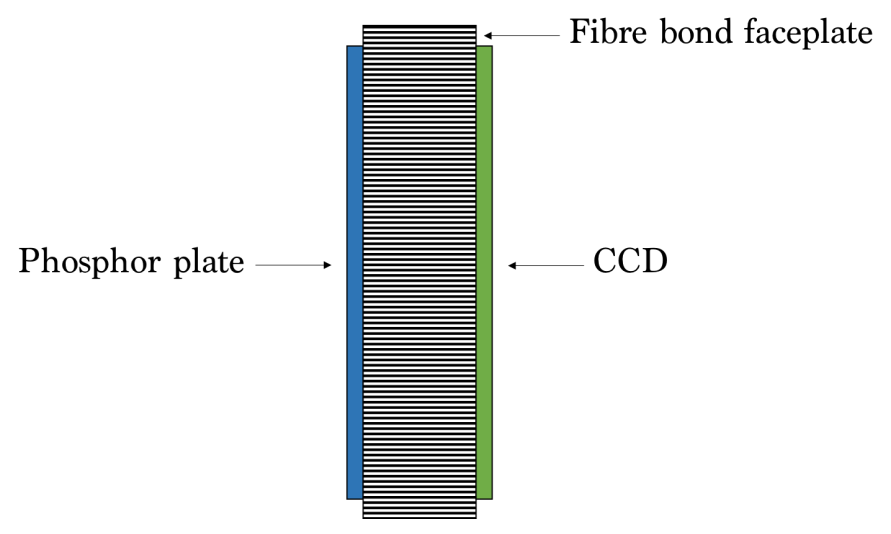

Figure 4.2: Fibre bond faceplate that sends the phosphor signal to the CCD.

mostly empty space and extremely fragile. The use of MCPs in framing cameras for soft X-ray detection and X-ray imaging has been commonly documented in recent decades [126, 127, 128, 129, 130].

Each microstrip corresponds to one 'frame' in the image and has a constant voltage bias of $\sim+200 \mathrm{~V}$ whereas the bottom of the MCP is 'grounded' (kept at zero potential), so that the electrons generated by X-rays arriving at the microstrips are not transferred to the bottom of the $\mathrm{MCP}$ and therefore do not generate any signal. The frame is activated by sending a negative voltage pulse $V(t)$ through the strip, so that then, the X-ray generated electrons cascade to the bottom of the MCP. As the electrons cascade and collide with the leaded walls of the pores, given that they are accelerating, more electrons are produced, and the signal is amplified (the signal amplification scales with $\sim V^{9}-V^{12}$ ). When the electrons reach the bottom of the MCP, they are further accelerated towards a phosphor plate kept at a constant voltage of $\sim+5000 \mathrm{~V}$. Each frame is activated by its own voltage pulse, which allows for activation at different times by delaying subsequent pulses.

For the particular case of the XRPINH used in this experiment, Sydor Framing Cameras were used, whose design is very similar to that described in reference [131]. A pinhole array is mounted on a snout in front of the framing camera, as depicted in Figure 4.1. This pinhole array creates sixteen images of the plasma, four in each 
microstrip. When a microstrip is activated, the pulse $V(t)$ takes $\sim 200$ ps to travel through the microstrip and this small delay allows for obtainment of images at different times within the same strip. This is the principle of operation of picosecond imaging framing cameras 132 .

The accelerated electrons impacting on the phosphor plate generate phosphorescent emission, which is transferred through a fibre bond faceplate onto a Chargecoupled Device (CCD) camera, where the image is recorded as shown in Figure 4.2 .

\subsubsection{Multi Purpose Spectrometer (MSPEC)}

The spectra for the experiment were obtained using framing cameras with MultiPurpose Spectrometer (MSPEC) snouts with elliptical crystals. In this geometry, the crystal is curved along one of its dimensions following an ellipse, whereas its other dimension is flat [133, 134]. This curvature provides a wide range of Bragg angles for X-ray diffraction. When the source (or effectively, a pinhole aperture) is placed at one focus $F$ of said ellipse, all the rays diffracting from the crystal will cross at the other focus, $F^{\prime}$, before getting to the detector. This particular characteristic allows one to place a second pinhole at $F^{\prime}$ to get rid of stray reflections, as shown schematically in Figure 4.3. The adaptation of the elliptical geometry to its use with framing cameras in the MSPEC is due to Heeter et al. [135] who did a thorough analysis of the materials and designs that could be used for an X-ray photon energy range between $\sim 0.4-8 \mathrm{keV}$, common in HED experiments.

The raw spectral data from the MSPEC are processed following the procedure in Marrs et al. [136]. In this particular experiment a pentaerythritol (PET) ellipse with a so-called E3 geometry was used, for which the crystal and MCP responses are known. The ellipse details for this geometry are given in Table 4.2, where the notation from [136] is used. With this notation $f$ is the distance between the two foci of the ellipse, $e$ is the eccentricity, $\eta$ is the angle between the instrument centreline 


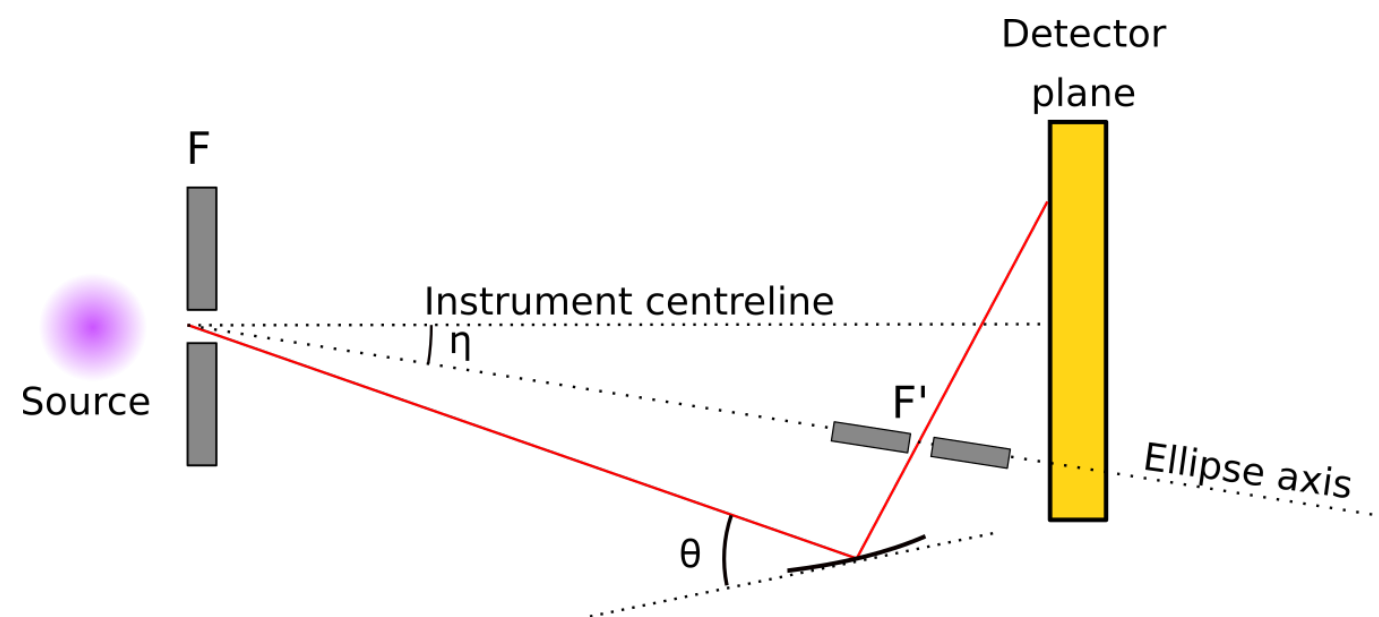

Figure 4.3: Schematic of the elliptical spectrometer design used for these experiments. When a pinhole aperture is placed at one focus $F$ of the ellipse, all the rays diffracting from the crystal will cross at the other focus, $F^{\prime}$, before getting to the detector. Placing a second pinhole at $F^{\prime}$, allows one to get rid of stray reflections.

and the ellipse axis and $\theta$ is the diffraction angle. Images on the detector lie within $\pm 15 \mathrm{~mm}$ of the spectrometer centreline.

For this diagnosis, instead of a CCD camera, the phosphor signal from the MCP is imprinted on photographic film for recording the spectrum. This choice was made because the CCD cameras available at OMEGA have some damaged pixels that fall within the spectral regions of interest. Furthermore, photographic film has a known response that is calibrated, and every dataset is accompanied by transmission data from a well-characterised optical wedge, which are imprinted upon the film next to the spectral data. However, an important caveat with using film is that it takes a long time to obtain a digitised signal that can be studied, as the film needs to be developed and scanned with a densitometer. This process can take up to several months due to the high volume of data that needs to be digitised and the scarcity of appropriate densitometers that can provide this service. For this reason, there

Table 4.2: Parameters for the geometry of the PET E3 ellipse used in the MSPEC.

\begin{tabular}{ccccc}
\hline \hline$f(\mathrm{~mm})$ & $e$ & $\eta\left(^{\circ}\right)$ & $\theta\left(^{\circ}\right)$ & Energy range $(\mathrm{eV})$ \\
\hline 310 & 0.9950 & 5.2 & $8.8-18.4$ & $4483-9280$ \\
\hline \hline
\end{tabular}


have been considerations to switch to using an Image Plate (IP) as a detector for the MSPEC data, but this idea was disregarded, as IPs present important variations depending on the scanner used [137, 138, and from IP to IP.

\subsection{Experimental set-up}

The experiments were performed at the OMEGA laser facility [35] at the LLE. The targets were tamped disk-shaped foils consisting of an inner foil made out of an iron and vanadium mixture, volumetrically equal; surrounded by a beryllium tamper. The inner sample material was $2000 \AA$ thick, $250 \mu \mathrm{m}$ in diameter. The outer tamper was $10 \mu \mathrm{m}$ thick ( $5 \mu \mathrm{m}$ on either side of the sample), with an outer diameter of $1000 \mu \mathrm{m}$. Having a larger tamper radius allows for a greater amount of Be outside the sample radius to provide radial confinement via thermal pressure [116]. The surface of the targets was smoothed to obtain a more uniform ablation surface which means the conduction layer will reach the sample material evenly and keep the sample temperature as uniform as possible, preventing hydrodynamic instabilities. 18 of the 60 OMEGA laser beams at $3 \omega$ were irradiated on each surface, with an intensity of $\sim 3 \times 10^{14} \mathrm{~W} \mathrm{~cm}^{-2}$ per side, or $\sim 6 \times 10^{14} \mathrm{~W} \mathrm{~cm}^{-2}$ when the laser burns through, with a total laser energy of $\sim 10 \mathrm{~kJ}$, delivered with a $3.0 \mathrm{~ns}$ square pulse. A schematic diagram of the experimental set-up is shown in Figure 4.4.

During the design of the experimental platform, two-dimensional HYDRA [57] simulations were run to estimate the degree to which the plasma would remain uniform. It was found that $\sim 2$ ns into the pulse, the plasma becomes underdense and the laser heats the entire plasma volume. At this point, the plasma becomes roughly isothermal, although with density variations in the Fe: $\mathrm{V}$ disk of up to a factor of $\sim 4$ mostly in the radial direction.

Time-resolved K-shell spectral emission data from the Fe:V plasma were obtained 

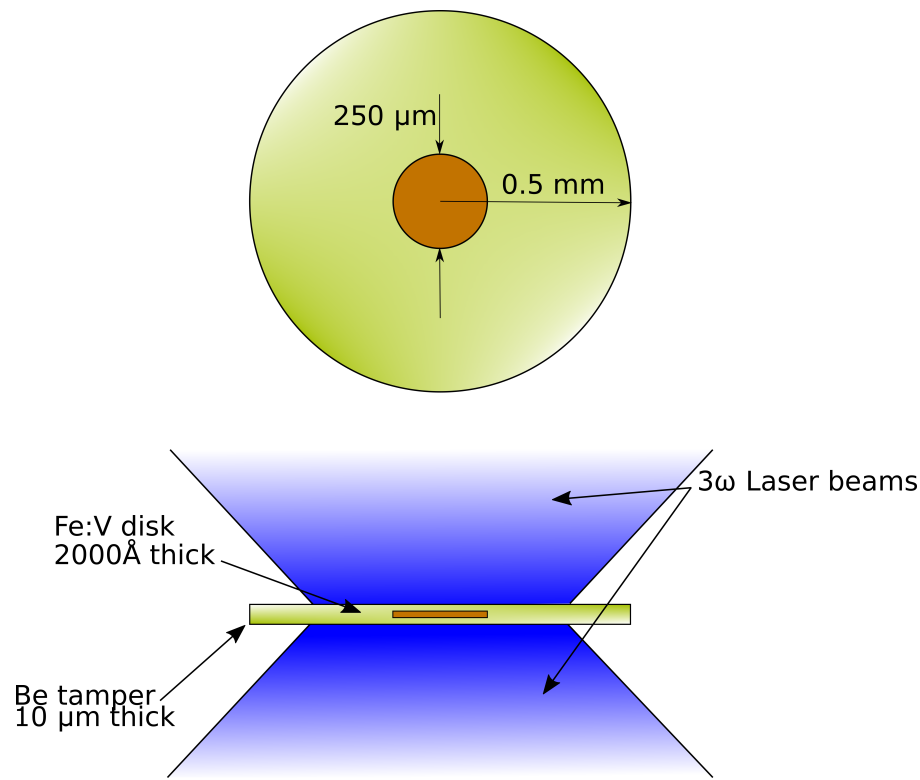

Figure 4.4: Front (top) and side (bottom) view of the targets. The $250 \mu \mathrm{m}$ diameter disk is a mixture of $\mathrm{Fe}$ and $\mathrm{V}$, volumetrically equal, tamped in a $10 \mu \mathrm{m}$ thick $1 \mathrm{~mm}$ diameter Be disk. 36 laser beams impinge upon the target, 18 on each side, heating the target up to $\sim 2 \mathrm{keV}$ in 3.0 ns. The thickness of the buried disk has been exaggerated for clarity.

using two MSPEC, each looking at the emission from either face-on or side-on view. These instruments obtained spectra at four time windows, each one integrated over $200 \mathrm{ps}$ and with a separation of $400 \mathrm{ps}$. The time-resolved axial and radial expansion was measured using two XRPINH, one for each view. The expansion data were obtained in sixteen $50 \mathrm{ps}$ time windows, grouped in four strips delayed $400 \mathrm{ps}$ from each other (as described in Section 4.1.2).

The OMEGA chamber has a truncated icosahedron geometry (football). Ports for diagnostics are at the centre of the flat hexagons or pentagons. There are six Ten-Inch Manipulator (TIM) ports, in which various cameras and snouts can be loaded, moved into the target chamber at desired distance from the centre, and pointed. Of the six TIM ports, four are in two pairs, such that the ports in each pair are opposing each other (TIM 2 and 3, and TIM 4 and 6). Each pair housed one framing camera with a MSPEC snout and one framing camera with a XRPINH snout, so both diagnostics had the same view. For the XRPINH ports, X-ray filters were selected such that 


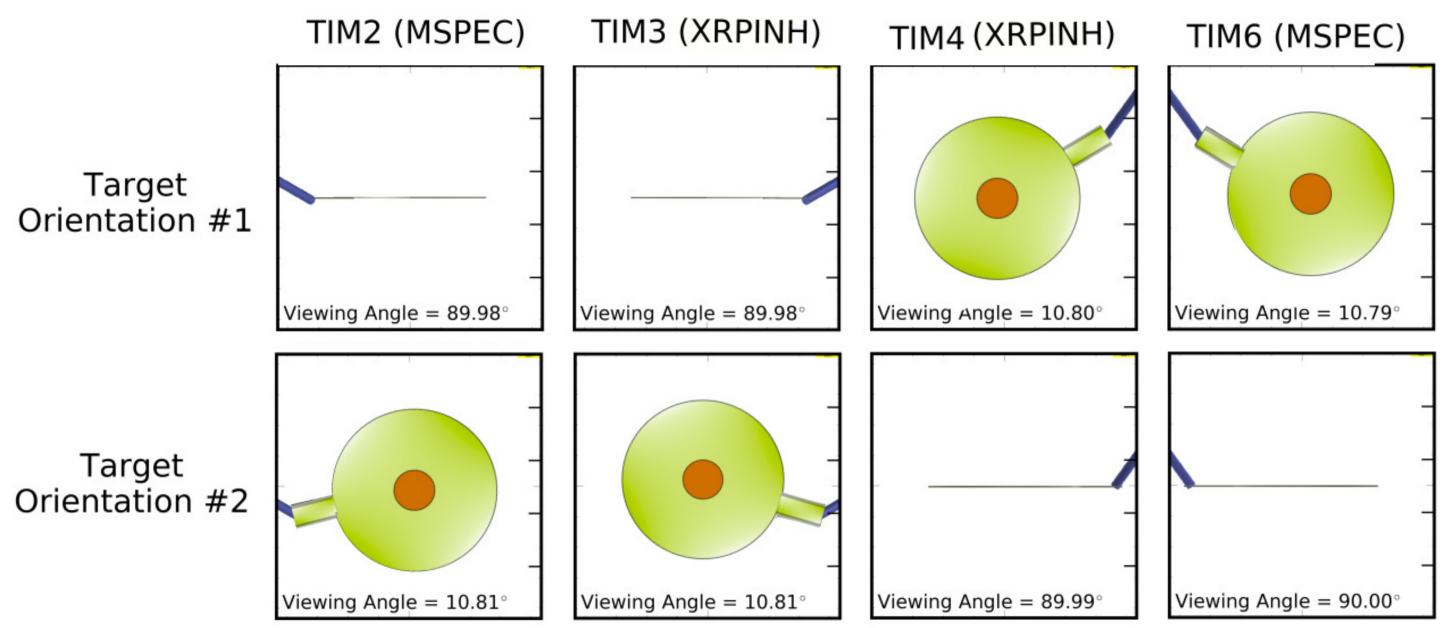

Figure 4.5: Different views for all crystal spectrometers (MSPEC) and pinhole imagers (XRPINH) for the different TOs. Each column corresponds to one of the TOs, and each row to a diagnostic. Each column corresponds to one of the TOs, and each row to a diagnostic. The indicated viewing angle is measured with respect to the target normal. Using this configuration it is possible to eliminate instrumental effects in the face-on and side-on data.

photon energies below $2 \mathrm{keV}$ were not detected, to minimise the signal from the Be tamper.

To minimise measurement uncertainties due to differences in individual diagnostic sensitivities, two Target Orientation (TO) configurations were used on alternating shots. This allowed each crystal spectrometer and pinhole camera to have both face-on and side-on views of the target, eliminating systematic errors due to crystal imperfections or framing camera sensitivities. At the same time, this configuration minimises the timing uncertainty, as the jitter for each MSPEC and XRPINH with respect to the start of the laser pulse is consistent, whereas it changes between diagnostics. The TOs were calculated such that in one case (TO 1) the target normal was perpendicular to TIMs 2 and 3, whereas in the other (TO 2) it was perpendicular to TIMs 4 and 6 . In both cases, the TO was such that the face-on view was as close to normal as possible $\left(10.8^{\circ}\right)$. The Target Normals $(\mathrm{TN})$ were calculated for each TO 

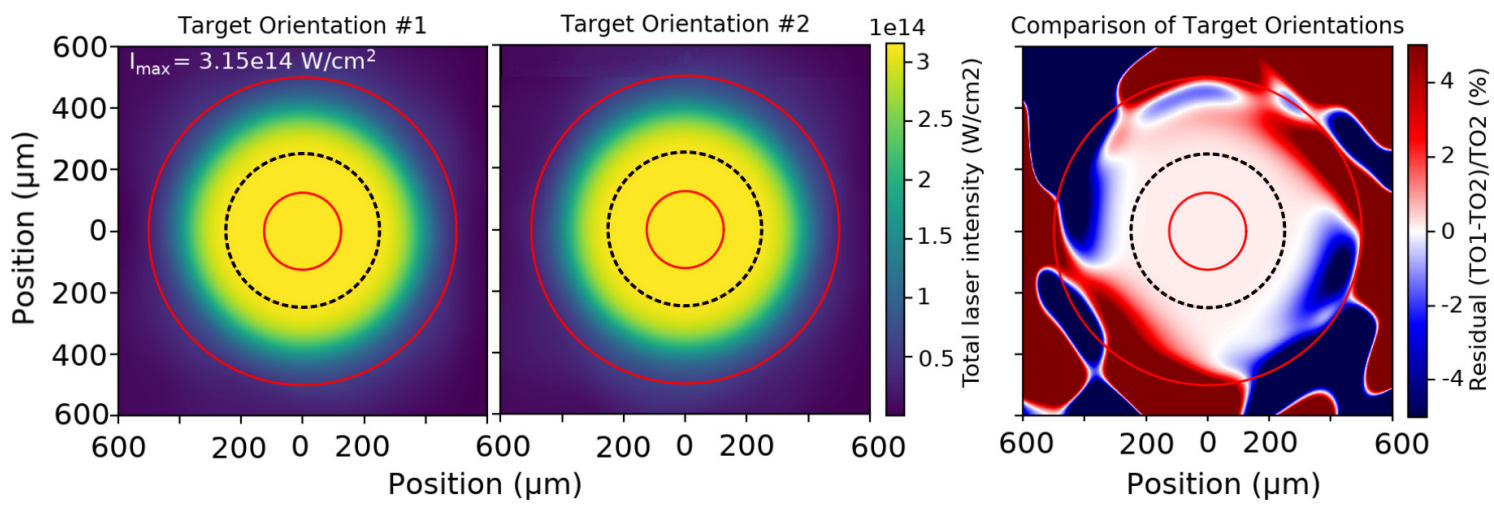

Figure 4.6: The centre and left plot show simulations of the laser drive on target for both TOs. The outer solid circle corresponds to the diameter of the Be tamper, whereas the inner solid circle corresponds to the diameter of the Fe: $\mathrm{V}$ disk. The laser profile was designed so that $90 \%$ of the laser intensity was deposited in a circle of twice the diameter of the buried disk (dashed black line) to minimise edge effects. The plot on the right shots the percentual residual between the drives for each configuration.

as

$$
\begin{aligned}
& \mathrm{TN}_{1}[\theta, \phi]=\left(\mathrm{T}_{2} \times \mathrm{T}_{4}\right) \times \mathrm{T}_{2}=\left[52.64^{\circ}, 341.98^{\circ}\right] \\
& \mathrm{TN}_{2}[\theta, \phi]=\left(\mathrm{T}_{4} \times \mathrm{T}_{2}\right) \times \mathrm{T}_{4}=\left[26.57^{\circ}, 161.97^{\circ}\right]
\end{aligned}
$$

where $\theta$ and $\phi$ are the polar and azimuthal angles of the OMEGA target chamber, and T2 and T4 are the diagnostic port orientations of TIM2 and TIM4 respectively (where the MSPEC were mounted). Comparison between the data from both MSPECs in each TO allowed to minimise the systematic error in the line ratios from the instrument response. Figure 4.5 shows the view of each instrument for both TOs.

The laser profile was designed so that $90 \%$ of the laser intensity was deposited on a circle with twice the diameter of the target disk, thus minimizing edge effects in the heating process of the plasma, and causing the tamper to provide the radial pressure needed for the radial confinement of the target. In order to maintain nominally similar on-target drive, beams were selected for each TO to match overall intensity and laser footprint, while also meeting facility beam safety requirements. For this purpose, the average incident beam angle with respect to target normal, as well as the variance in incident beam angle for each $\mathrm{TO}$ were matched. Calculations prior to the experiment 


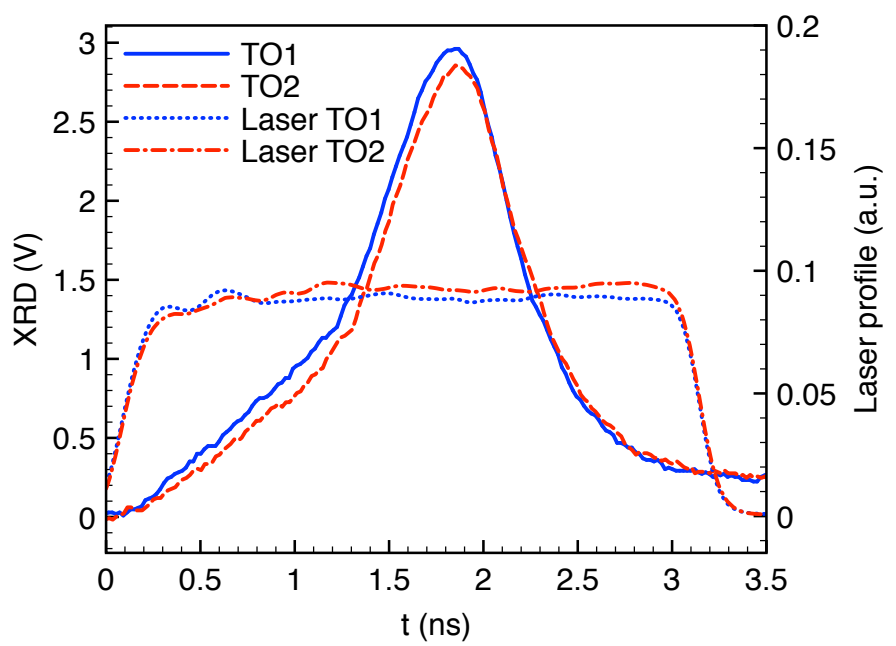

Figure 4.7: Comparison between the total power (in units of voltage measured by DANTE) emitted by the plasma in the $1.3-1.5 \mathrm{keV}$ photon energy range for both TOs. It can be seen that in both TOs, the emitted power is very similar, which is indicative of the fact that the shots are equivalent and therefore, they can be compared. The x-axis corresponds to the time with respect to the start of the laser pulse. The $\mathrm{y}$-axis shows the signal measured by the X-ray Diode (XRD) in Volts. The laser profile for both TOs is also shown in arbitrary units for comparison.

predicted an agreement between the nominal drive for both TOs within $0.16 \%$. The laser configuration for each TO, as well as the residual between them are shown in Figure 4.6. In this figure, the inner solid red circle represents the target disk, whereas the dashed line corresponds to twice its diameter. The plot to the right of Figure 4.6 shows the percentual difference in laser intensity between both views, where it can be seen that there are no significant differences within the target disk.

\subsubsection{Validation of target orientation}

The consistency between the two TOs was checked experimentally using two diagnostics. A p510 optical streak camera measured the total power profile and indicated that it was similar for both TOs, given shot to shot variation, with peak differences of less than $\pm 20 \%$.

A second consistency check was done using the spectrometer Dante by comparing the absolute flux of soft X-rays emitted by the target in both TOs. Good agreement 
(within 10\%) was found at all times during the emission, thus showing that both laser configurations drive the targets in a similar way. Figure 4.7 shows an example of this comparison, corresponding to the signal measured by the channel 9 of Dante $(h \nu=$ $1.3-1.5 \mathrm{keV}$ ), which measures the L-shell emission of the plasma, after correcting for the channel response. It can be seen that in both TOs, the emitted power is very similar, which is indicative of the fact that the shots are equivalent and therefore, they can be compared. The $\mathrm{x}$-axis corresponds to the time with respect to the start of the laser pulse. The y-axis shows the signal measured by the XRD in Volts. The laser profile for both TOs is also shown in arbitrary units for comparison

\subsection{Experimental results}

High quality spectra were obtained from these experiments. Figure 4.8 shows the MSPEC and XRPINH data from two different shots representing an experimental "pair" of shots which had identical drive parameters with the exception of TOs. The left two columns are side-on data from TO 1 while the right two columns are face-on data from TO 2. The MSPEC data shown in columns 1 and 3 in Figure 4.8 are consequently taken from the same diagnostic meaning the diagnostic timing with respect to the start of the driver beams is nominally identical. Additionally, since the same MSPEC is shown in both columns, the crystal reflectivity and overall diagnostic response is identical making direct comparisons between them straightforward. Likewise, the XRPINH data shown in columns 2 and 4 are also from the same diagnostic and therefore the target geometry from face-on and side-on views are shown at identical times with respect to the start of the laser pulse for each respective shot. The spectra are normalised to the peak of the $y$ line, as the detector is not absolutely calibrated. The imaging data are normalised to the peak brightness at every time step. The red dashed lines represent the measured limits of the plasma in both dimen- 
Side-on
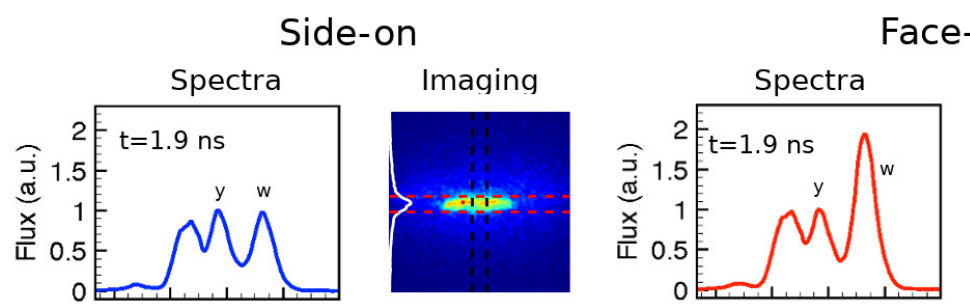

Face-on
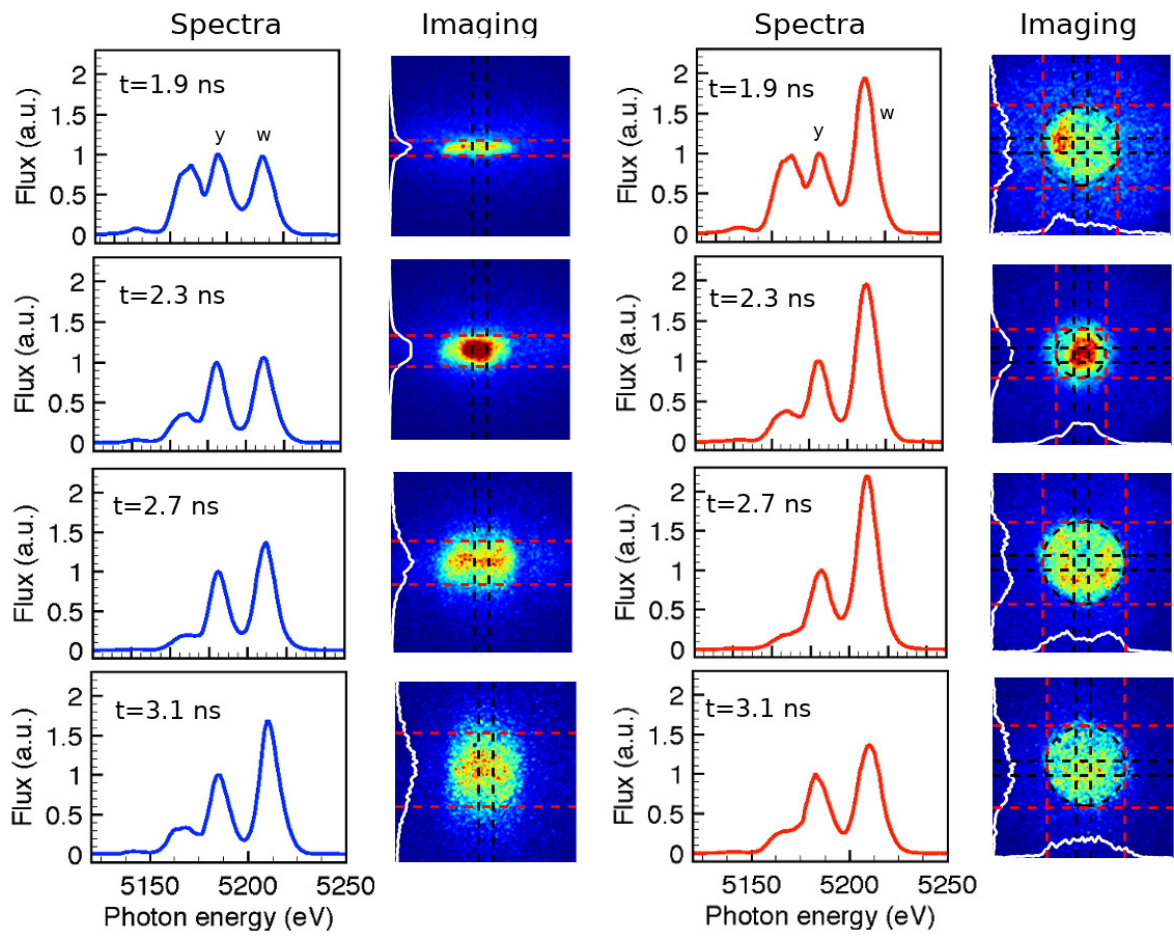

Figure 4.8: Spectral and imaging data for side-on and face-on views. Each row represents a timestep in the plasma expansion, with time going from top to bottom. The spectra are normalised to the peak of the $y$-line. The bumps on the low-energy wing of the $y$-line correspond to the Li-like satellite emission. In the imaging data, the red dashed lines represent the measured limits of the plasma in both dimensions. The white lines show the lineouts through the centre of the images, whereas the dashed black lines represent the regions used to integrate those lineouts. For the face-on view, an additional black dashed line shows the circle that was fit to the data.

sions. The white lines show the lineouts through the centre of the images, whereas the dashed black lines represent the regions used to integrate those lineouts. For the face-on view an additional black dashed line shows the circle that was fit to the data.

The rows in Figure 4.8 correspond to different frames for all diagnostics, with time going from top to bottom. The first row was taken at 1.9 ns after the start of the driver beams, the second row at $2.3 \mathrm{~ns}$, the third at $2.7 \mathrm{~ns}$ and the fourth at $3.1 \mathrm{~ns}$, just after the laser is turned off (at $3.0 \mathrm{~ns}$ ). In the XRPINH data there is a density gradient in the radial direction, which is most intense between the first and the second rows. This behavior is in agreement with the density gradients predicted 


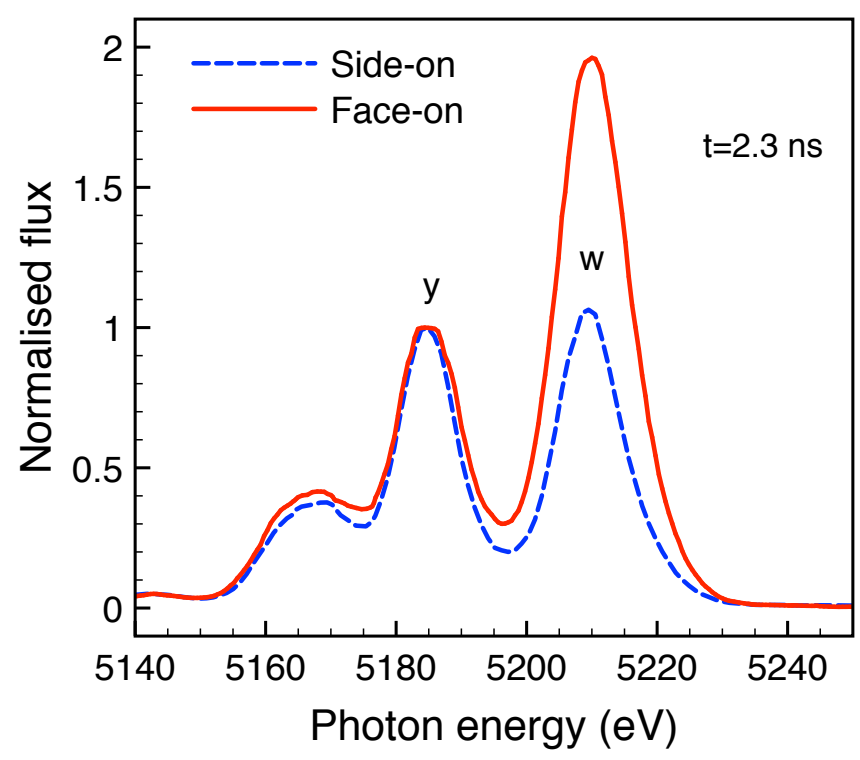

Figure 4.9: Example of face-on and side-on spectra for $\mathrm{V}$, taken from Figure 4.8. These were obtained by the same spectrometer in two subsequent shots using the two different TOs. The spectra are normalised to the peak of the $y$-line (photon energy $\sim 5185 \mathrm{eV})$.

by the hydrodynamic simulations.

The iron emission is not accounted for here, as there was a significant amount of iron in the Be tamper, which blends with the emission from the disk. The spectral data in columns 1 and 3 show only the Vanadium $\mathrm{He}-\alpha$ complex. Since the $y$ line $(\sim 5185 \mathrm{eV})$ is optically thin, whereas the $w$ line $(\sim 5210 \mathrm{eV})$ is not, it is possible to quantify the anisotropy effect due to target geometry by comparing the $w / y$ ratio at each time.

It can be seen that the spectra from face-on and side-on view differ in the relative intensity of the $w$ and $y$ lines, whereas the relative intensity of the $y$ line and the Li-like satellite peak $(\sim 5165 \mathrm{eV})$ is independent of the view. Figure 4.9 shows a particular example of these data, corresponding to the second row in Figure 4.8. The spectra were taken at $t=2.3 \mathrm{~ns}$ after the start of the pulse, and the aspect ratio $H / R$ at that time is $1.0 \pm 0.1$. Here, the $w$ line is significantly more intense than the $y$ line for the face-on view (column 3 in Figure 4.8), whereas in the side-on view (column 1) 
both lines are comparable. This is indicative of the fact that the $w$ line is enhanced along the shortest escape path (face-on) and reduced along the longest (side-on). As the aspect ratio increases (third and fourth rows in Figure 4.8) this effect becomes less important and the differences between both views are reduced.

It was predicted by the hydrodynamic simulations, and later confirmed by the Xray imaging data, that for the last timestep (3.1 ns), the target is no longer confined by the beryllium tamper and expands freely, therefore losing its cylindrical geometry. For this reason, for the remainder of this chapter, data from only the first three timesteps is presented.

\subsubsection{Target expansion}

The size of the plasma was obtained from the XRPINH images (second and fourth columns in Figure 4.8). Lineouts through the centroid of each image were fitted to supergaussians. A combined analysis of the Full Width at Half Maximum (FWHM) and the second derivative of these supergaussians was used to obtain a measurement of the plasma extension in both directions. For the face-on images, two perpendicular lineouts were taken to obtain an average radius (shown in Figure 4.8 as a black dashed circle).

The measured dimensions of the plasma during the expansion are plotted in Figure 4.10. It was found that the radius stays constant within $20 \%$, showing that the large radius of the Be tamper compared to the Fe: $\mathrm{V}$ sample is confining the plasma as expected. The target is therefore mostly expanding in the axial direction. The expansion velocity was measured to be $v=206 \pm 7 \mu \mathrm{ms}^{-1}$, which corresponds to each face of the cylinder moving at half this value $\left(v_{\text {face }}=103 \pm 4 \mu \mathrm{ms}^{-1}\right)$.

The aspect ratio $H / R$ was calculated from the data in Figure 4.10. As the integration window for the XRPINH cameras is $50 \mathrm{ps}$, and for the MSPECs it is $200 \mathrm{ps}$, the average target size in each MSPEC time window was used for the spectral analysis. 


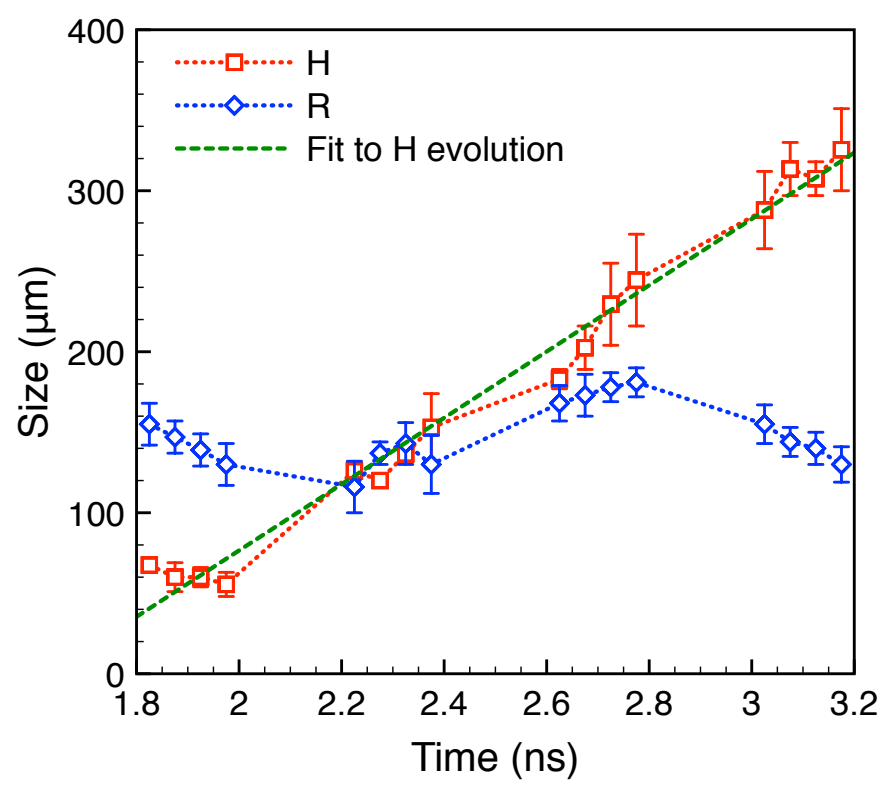

Figure 4.10: Size evolution of the targets in both the axial $(\mathrm{H})$ and radial $(\mathrm{R})$ dimensions. The velocity of the expansion is obtained from a linear fit to the axial expansion (dashed green line), obtaining a value of $206 \pm 7 \mu \mathrm{ms}^{-1}$

\subsubsection{Plasma conditions}

To estimate the plasma temperature from the spectra, each experimental spectrum is fitted to a combination of Cretin spectra for different temperatures using a genetic algorithm [117]. Initially, the algorithm creates several random temperature distributions and selects those which fit the spectrum best. The next iteration of the algorithm takes the best combinations from the previous iteration, and combines them (hence the name 'genetic'). Every iteration, the newly generated combinations, or offspring, have one of their parameters randomly changed, or 'mutated', and additional random distributions are introduced. By allowing the algorithm to iterate several thousands of times, the temperature distribution eventually converges to the best possible fit to the data. To avoid any optical depth effects affecting the result, an additional parameter was introduced in the fit, multiplying the $w$ line (only optically thick component of the spectra) in order to reproduce the experimental data. This is shown in Figure 4.11. 4.11a shows an example of a fit obtained for the K-shell 


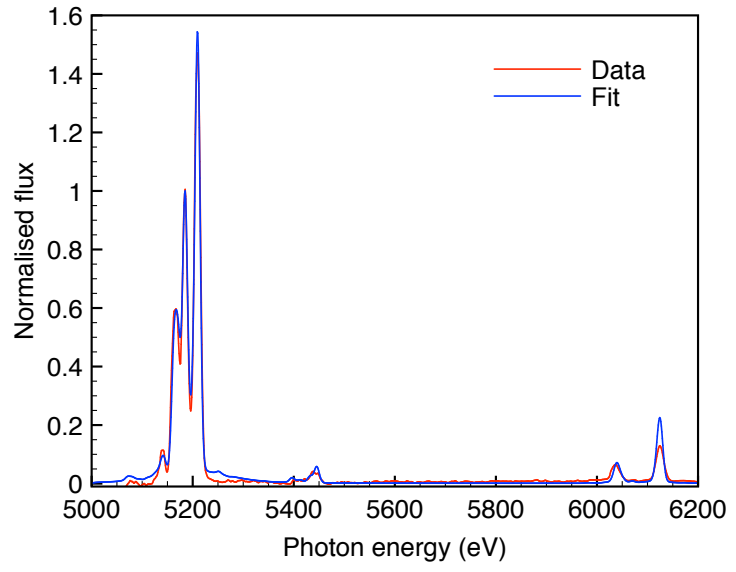

(a)

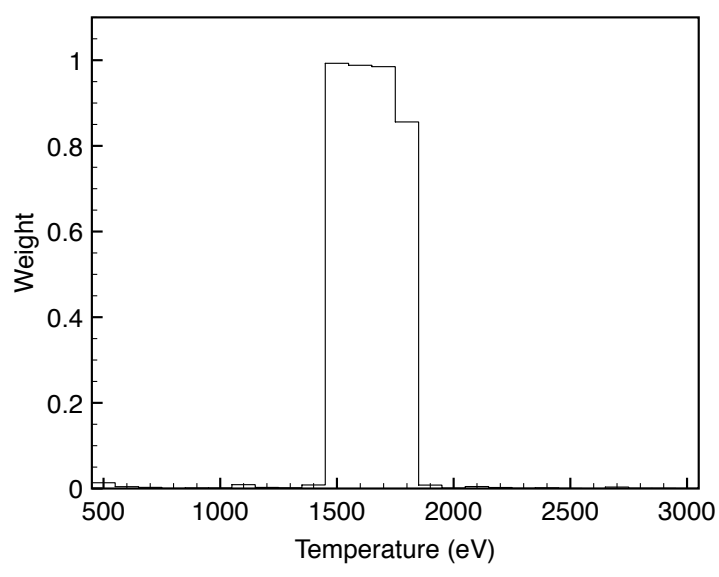

(b)

Figure 4.11: Example of the results from the genetic algorithm used to estimate the plasma temperature. (4.11a) Comparison between experimental spectrum and the result from the fit. (4.11b) Distribution of temperatures in the plasma obtained from the fit.

spectrum of vanadium, whereas the temperature distribution corresponding to that fit is shown in 4.11b. Note how, even though the temperature distribution is initialised as random, the final temperature distribution is relatively narrow. For the fit, temperatures from 500 to $5000 \mathrm{eV}$ were included, but the range in the figure has been limited for simplicity. The results for the plasma temperature distribution are shown in Figure 4.12a, where the error bars include the width of the temperature distributions calculated and their variability among shots and MSPEC.

The ion density of the plasma was calculated using the size of the plasma measured from the XRPINH images and imposing a conservation of particles condition. The results for the ion density of vanadium are indicated in Figure $4.12 \mathrm{~b}$ as green circles. The error in these data comes from the resolution of the system (due to the pinhole size, diffraction and point-spread function of the framing cameras), as well as from differences between shots and cameras. The ion density of this element was also obtained from the $w / y$ ratios for face-on and side-on view, using the method described in Section 3.4 [55]. The results are shown in Figure $4.12 \mathrm{~b}$ as the blue triangles. The error values arise from the error in the line ratios (shown later in Figure 4.14). It can 


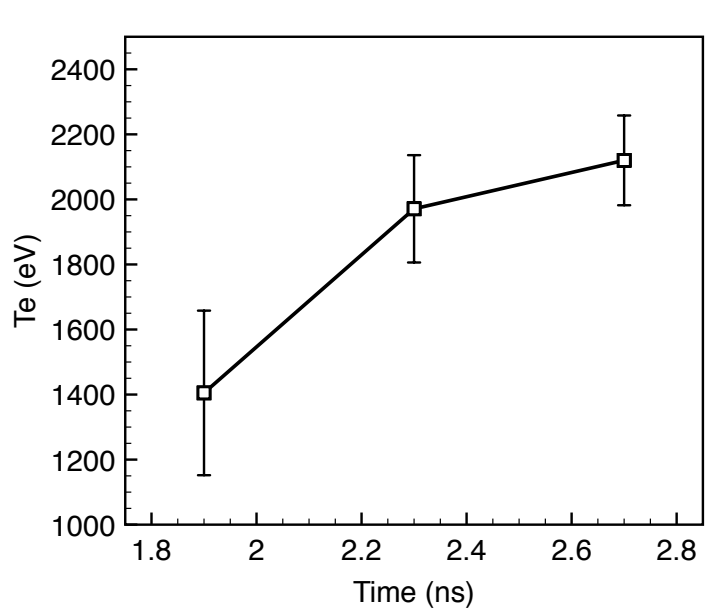

(a)

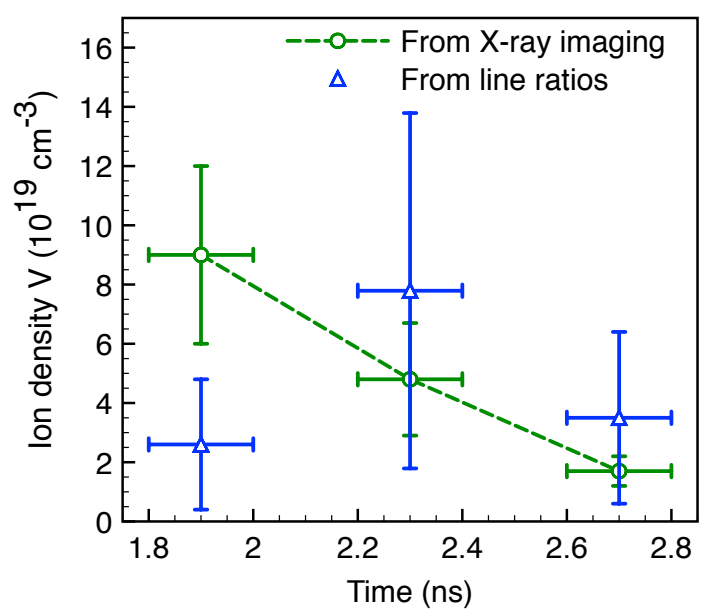

(b)

Figure 4.12: 4.12a) Temperature obtained from the genetic algorithm fit for both spectrometers. (4.12b) Density of Vanadium ions in the targets measured by two different methods.

be seen how for the second timestep, where the density gradient is most important, the error in the density is maximum, as the assumption of a uniform plasma does not hold. Although the spectral method does not show a clear trend for the density, which is observed in the imaging data, it retrieves the right order of magnitude.

\subsection{3 $w / y$ line ratios}

To understand the effects of the geometry on the spectra, the plasma was simulated using the Cretin model described in Chapter 3. This model was used for two things - to obtain the theoretical $w / y$ line ratio for a perfect cylinder at the experimental conditions, and to model the Li-like satellite contribution, so that it can be removed from the data. An experimental $w / y$ ratio was then obtained from the data by integrating the flux under the remaining $\mathrm{He}-\alpha$ lines. It was found that density gradients of the order of those predicted by HYDRA had a negligible effect in the line ratios at the conditions of interest. Figure 4.13 shows an example of how this ratio is obtained for face-on and side-on emission. 


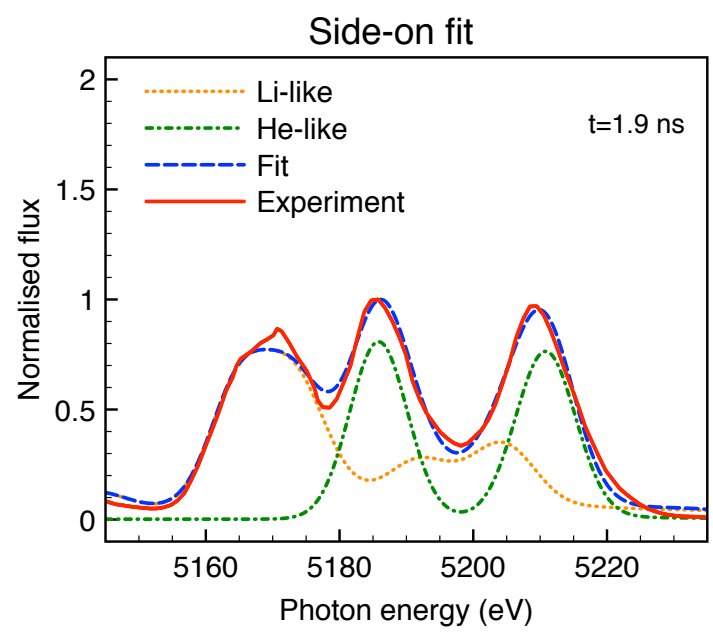

(a)

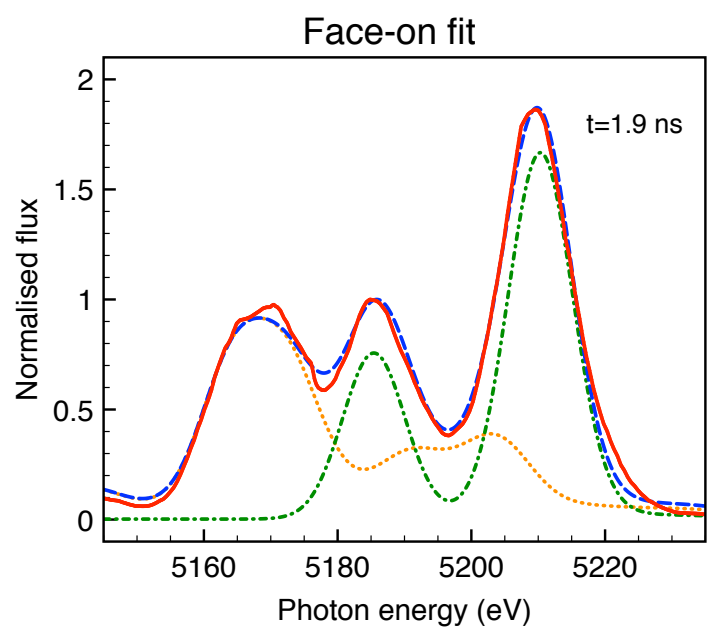

(b)

Figure 4.13: Example of face-on and side-on fits to the spectra at $t=1.9 \mathrm{~ns}$, where the Li-like contribution has been obtained from the Cretin model. The $w / y$ ratio was obtained by integrating the emission under each of the He-like lines.

Figure 4.14 shows the comparison between the $w / y$ ratios for face-on and sideon view, as measured in the experiment. The red squares correspond to the ratio measured for face-on view, whereas the blue circles correspond to the side-on view results. The error bars correspond to shot-to-shot differences, as well as the differences between the two spectrometers. The black lines (solid and dashed) correspond to the results obtained for a perfect cylinder using the Cretin model. The solid line corresponds to the face-on view prediction while the dashed line corresponds to the side-on view. The error bars in the model correspond to the uncertainty in the temperature and density of the plasma. For comparison, the results predicted for a spherical plasma at the same temperature and density are also shown (gray shaded region). In this case, there are no differences between views, and this region lies between the prediction for face-on and side-on views of a cylinder.

Note how early in the expansion, for small aspect ratios, the obtained ratios agree well with the model predictions for a perfect cylinder. As the aspect ratio grows and the plasma expands, the geometry starts to differ from a perfect cylinder (as it can 


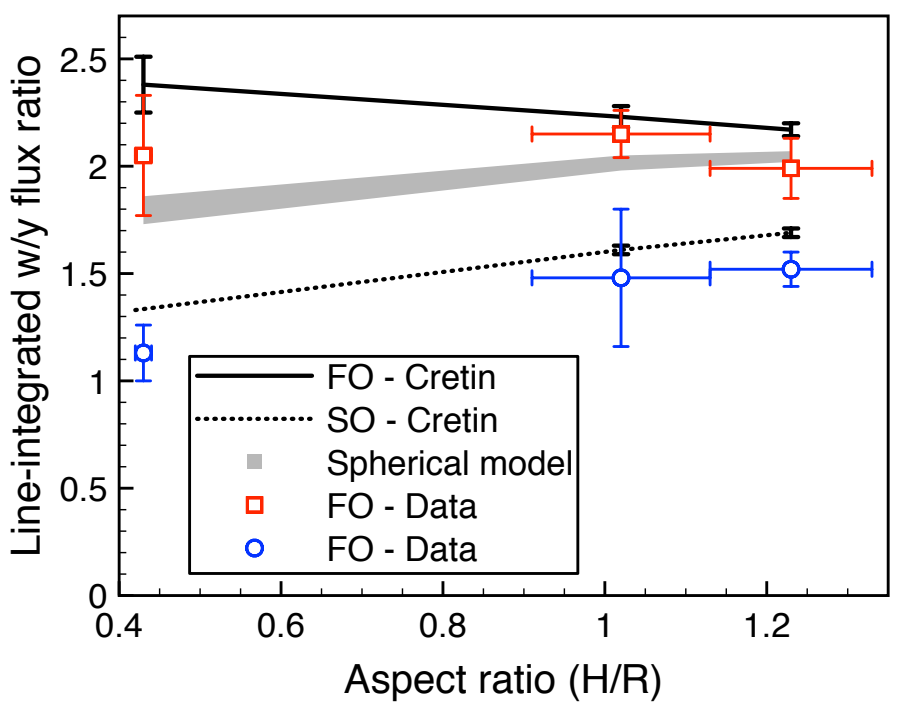

Figure 4.14: Line-integrated $w / y$ flux ratio from experiment and simulations as a function of the aspect ratio $(H / R)$ of the plasma. The red squares correspond to the face-on view experimental results, whereas the blue circles represent those obtained from a side-on view. The horizontal error bars arise from the error in the plasma dimensions, and the vertical bars represent the shot-to-shot variations in the line ratio. The black lines correspond to the line-integrated line ratios obtained from the Cretin model for a cylindrical plasma (the solid and dashed lines correspond to faceon and side-on view spectrometers respectively). The vertical error bars in the model arise from the uncertainty in the temperature and density of the plasma. The gray shaded region corresponds to the line ratios obtained using a spherical plasma model.

be seen in Figure 4.8 and therefore the experimental results start differing from the model.

\subsubsection{Statistical significance}

The data show a clear difference between the spectra for face-on and side-on views. To measure the strength of this evidence, two different statistical tests were performed on the data. First, a t-test, in which the null hypothesis was that no differences are present between the $w / y$ ratios for face-on and side-on view. This test retrieved a probability of $p=6 \times 10^{-4}$ of obtaining the experimental data if the null hypothesis were true, which indicates strong evidence that enhancement and suppression of the optically thick line were observed. 
Secondly, a Bayes factor was calculated for the cylindrical radiative transfer model with respect to the spherical plasma model, given the experimental data. Bayes factors indicate the preference for one model with respect to another given a particular dataset [139, 140, 141]. They can be calculated as

$$
K=\prod_{i=1}^{N} \frac{\exp \left[-\left(\frac{d_{i}-m_{1 i}}{\sigma_{i}}\right)^{2}\right]}{\exp \left[-\left(\frac{d_{i}-m_{2 i}}{\sigma_{i}}\right)^{2}\right]}
$$

where $d_{i}$ represents the $w / y$ ratio of each data point, and $m_{(1,2) i}$ the corresponding value from both models being compared. The value of $\sigma_{i}$ is the standard deviation from the data, and $N$ is the total number of data points. For each model the most favorable value within the error bars was taken to calculate $K$.

For the data in Figure 4.14 a value of $\log (K) \sim 24$ is obtained. This corresponds to a 'decisive' preference for the cylindrical model with respect to the spherical plasma, according to both the interpretations of Jeffreys [140], and Kass and Raftery [141], corresponding to the maximum degree of certainty achievable in both cases. It is interesting to note that this value is heavily weighted by the side-on data, as for the face-on view there is not a clear preference for a particular model. However, the cylindrical model can reproduce the face-on and side-on data at the same time, whereas the spherical model cannot, making it the preferential model for this case.

\subsection{Validation of atomic models}

Most experiments that aim to measure the opacity of a plasma use either transmission [142] or emission [143] measurements. However, this platform can be used to obtain information about the plasma opacity just from the geometry of the target. As mentioned in Section 4.3.2, this platform can be used to test the density calculation method described in Chapter 3. In addition, given that in this particular platform 
the dimensions of the plasma were measured, it is possible simplify the calculations and obtain an absolute value of the opacity at the centre of the $y$ line of vanadium, $\kappa_{0}^{y}$, at the experimental temperatures and densities. This can easily be seen by taking the ratio of the $w$ and $y$ lines at line centre (for simplicity), for both face-on and side-on view. At line centre, the $w$ line is completely optically thick $\left(1-e^{\tau_{w}} \sim 1\right)$, and therefore its emission can be simplified, yielding

$$
\frac{F_{w} /\left.F_{y}\right|_{\text {face }}}{F_{w} /\left.F_{y}\right|_{\text {side }}} \sim \frac{1-e^{-1.45 \kappa_{0}^{y} R}}{1-e^{-\kappa_{0}^{y} H}},
$$

where the only unknown is $\kappa_{0}^{y}$. This equation can now be solved using the experimentally measured line ratios and plasma dimensions. The results are shown as the red shaded region in Figure 4.15 .

Figure 4.15, also shows the results obtained for the opacity at line centre of the $y$ line at the same plasma conditions for three different codes: Cretin [31], SCRAM [112] and FLYCHK [144]. It is interesting to note that these codes predict very different values for the opacity for a fixed set of experimental conditions.

From Equations 3.16 and 3.17 and the definition of optical depth, it follows that the opacity of a given transition at line centre is given by

$$
\kappa_{0}=n_{i} F \frac{\pi e^{2}}{m_{e} c} f \phi_{0}
$$

For a fixed temperature and density, the only variables in Equation 4.4 are $F$ and $f$. It was found that in the experimental conditions all three codes calculate a similar $F$ for the $y$ line (that is the fraction of ions in the He-like ground state). However, the codes present differences of up to $\sim 50 \%$ in the value of the oscillator strength of the transition. This differences cause the calculated opacity of the line to differ greatly between codes and from the experimental data. While Cretin and SCRAM have a similar atomic model, and therefore give a similar answer for the opacity of the 


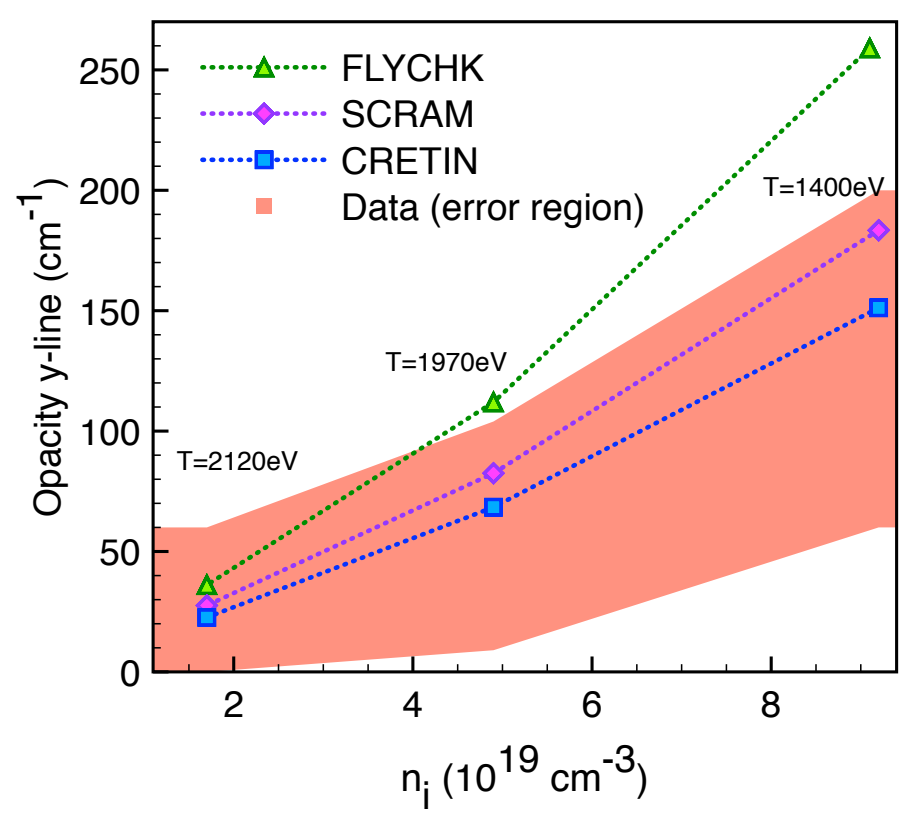

Figure 4.15: Value of the opacity of the $y$ line at line centre as calculated from the experimental line ratios compared with the results from three different codes.

line, FLYCHK uses a scaling expression for the oscillator strength of the same line in different elements which overestimates the strength of some transitions (including this one). If the value of the oscillator strength is corrected, using the numbers from the NIST database, the difference between FLYCHK and the other codes is reduced to $\sim 10 \%$.

This result shows how differences in the atomic models used in different codes can produce important differences when calculating properties of the plasma, such as opacity. Using experimental values to benchmark these values can help discriminate between atomic models.

\subsection{Influence of the pulse shape}

For one particular shot in the experiment, a different pulse shape was used. The new pulse shape consisted on a 2.6 ns square pulse, preceded by a low-intensity picket, fired 1 ns before (hereafter referred to as the 'picket' pulse). A comparison between 


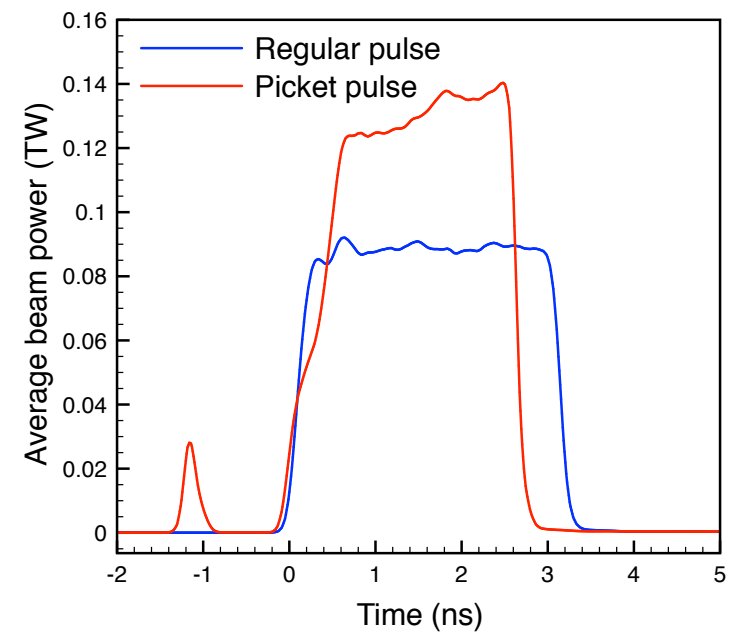

(a)

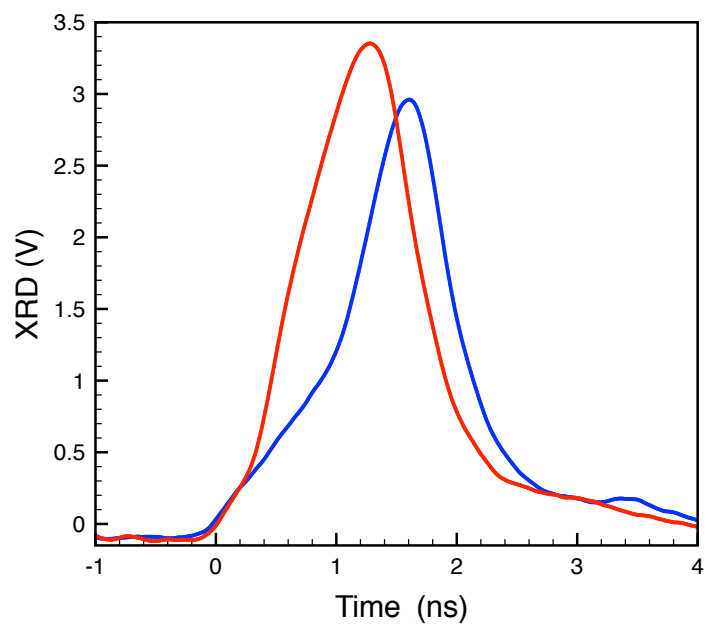

(b)

Figure 4.16: 4.16a Comparison between the two laser pulses used in the experiment. (4.16b) Comparison between the total power emitted by the plasma in the $1.3-1.5 \mathrm{keV}$ photon energy range for both pulse shapes.

the two laser pulses is shown in Figure 4.16a, The obtained results are compared in this section with those from the 'regular' square pulse.

\subsubsection{X-ray emission}

The idea behind the 'picket' pulse was to check whether an initial impulse - the picket

- would make the plasma absorb the laser energy more efficiently. This was checked by looking at the Dante data for the picket pulse shot, and comparing it with the shots which used the square pulse with the same TO. Figure 4.16b shows the voltage measured by Dante's channel $9(1.3-1.5 \mathrm{keV})$ for both cases. It can be seen how for the picket pulse the emission rises faster, as the instantaneous power is greater than in the case of the single square pulse.

To compare the efficiency of the laser energy absorption in the plasma, the total emission measured by DANTE for all channels was time-integrated and normalised to the total energy in the laser pulse. Figure 4.17 shows the ratio of the normalised intensity in the regular pulse to the picket pulse for all active channels (channels 12 


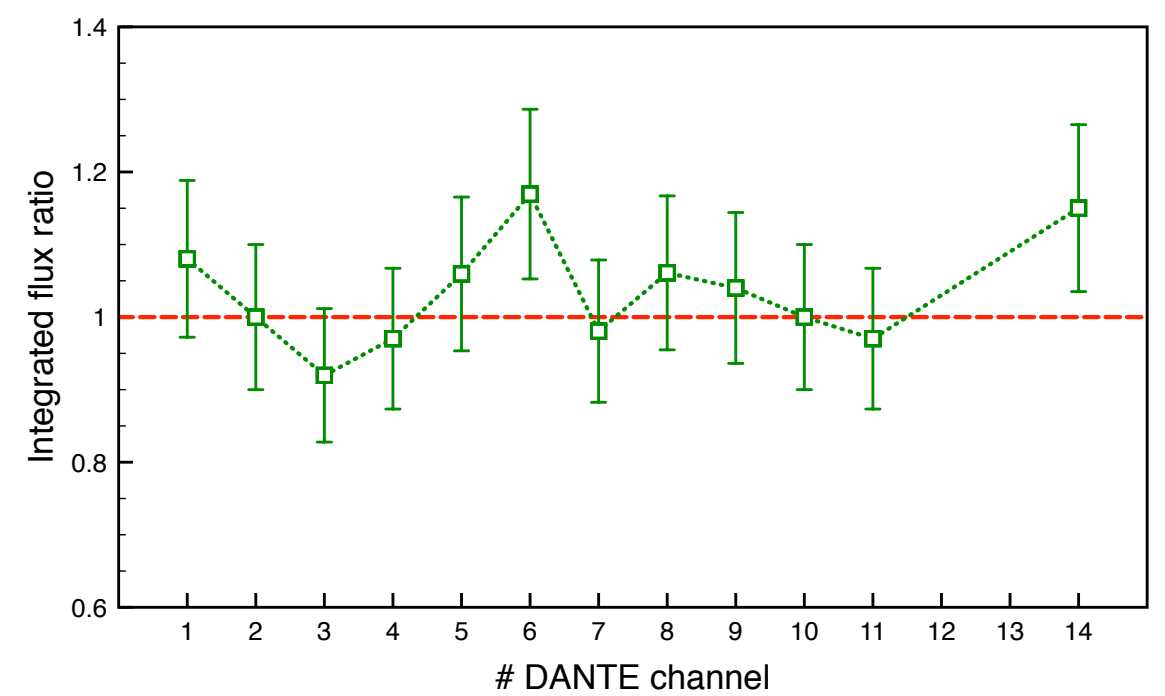

Figure 4.17: Ratio of the integrated radiation flux (normalised by the pulse energy) emitted by the plasma using a square laser pulse with respect to the picket pulse for each active DANTE channel. The DANTE channels are numbered as indicated in Table 4.1. The dashed red line indicates a constant value of 1 , which corresponds to no differences in the efficiency of the laser absorption between the two pulse shapes. The vertical error bars correspond to $10 \%$ of the measured ratios, to account for differences between TOs.

and 18 have not been included as the signal-to-noise ratio in them is too low). The error bars indicate $10 \%$ of the ratio, which is an estimate of the difference in radiation intensity between the different TOs.

It can be seen that the difference between both pulses is negligible, as the ratio is roughly constant and equal to one across all energy ranges (the energy range for each Dante channel was indicated in Table 4.1).

\subsubsection{Expansion of the target}

The two main differences between the picket and the regular pulse (picket arriving 1 ns earlier than main pulse and power being greater in the case of the picket) cause the expansion of the plasma in both the axial and radial directions to be different in both cases. Figure 4.18 shows a comparison between the time evolution of the thickness and radius of the plasma (as well as its aspect ratio) for both the picket 


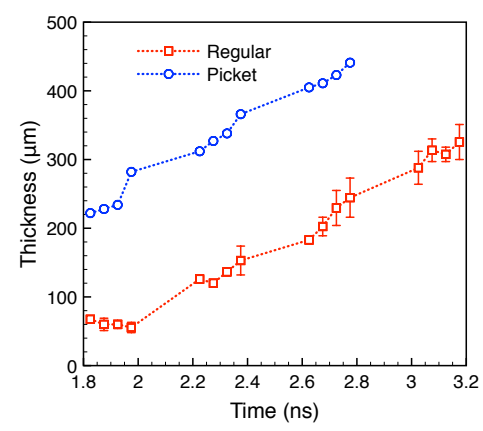

(a)

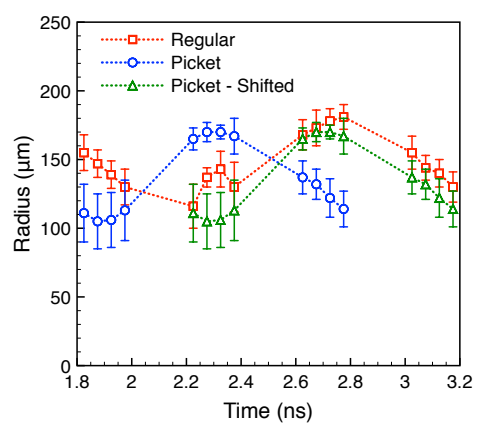

(b)

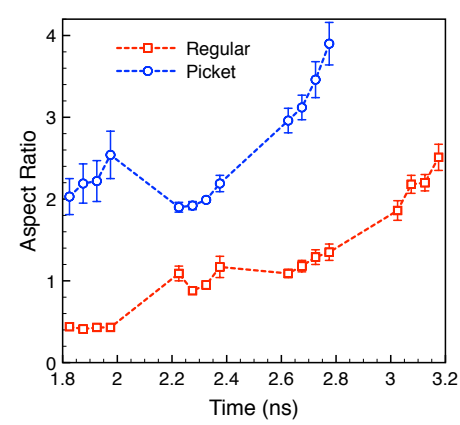

(c)

Figure 4.18: Expansion data from the picket pulse compared with the regular pulse. (4.18a) Thickness of the target in the axial direction. 4.18b Radius of the target as a function of time. The green triangles correspond to the data from the picket pulse shifted by $0.4 \mathrm{~ns}$, showing that the radial expansion follows a similar trend regardless of the pulse shape. 4.18c Aspect ratio of the plasma cylinder as a function of time.

(blue circles) and the regular pulse (red squares).

In the picket case, the thickness of the target (Figure 4.18a) grows faster than in the case of the single square pulse. This is due to the fact that the laser power is greater for the picket, and therefore the plasma gets hotter and the ions move faster. Fitting the thickness data to a line it is possible to estimate a velocity of $\sim 226 \pm 9 \mu \mathrm{m} \mathrm{ns}^{-1}$ for the picket case. The expansion velocity for the square pulse was fitted as $\sim 206 \pm 7 \mu \mathrm{ms}^{-1}$, indicating an $10 \%$ increase in the picket case. This is not surprising, as the intensity of the picket pulse is $\sim 55 \%$ greater than that of the square pulse, and the absorbed laser intensity and the plasma temperature are related by [145]

$$
I_{a b s} \propto T_{e}^{3 / 2}
$$

Assuming that the electrons and the ions are at the same temperature and taking into account that $T_{i} \propto v^{2}$,

$$
\frac{v_{\text {picket }}}{v_{\text {regular }}}=\left(\frac{T_{e}^{\text {picket }}}{T_{e}^{\text {regular }}}\right)^{1 / 2}=\left[\left(\frac{I_{\text {abs }}^{\text {picket }}}{I_{\text {abs }}^{\text {regular }}}\right)^{2 / 3}\right]^{1 / 2} \sim 1.55^{1 / 3} \sim 1.16
$$


which is in good agreement with the measured difference in velocities from the picket to the regular pulse. This result is an additional indication that the laser absorption in the plasma is proportional to the laser intensity, showing that the picket pulse is not driving the plasma more efficiently than the regular pulse.

The pulse shape also causes the radial expansion to be different. Although in both cases the radius follows a similar trend, initially contracting to later expand, in the picket case this trend starts earlier, which is likely to be due to the delay between the picket and the main pulse. In Figure $4.18 \mathrm{~b}$, the green triangles correspond to the radius of the plasma in the picket pulse, shifted by 0.4 ns later in time to compare with the regular pulse data.

Due to the combination of the axial and radial expansion being different in the picket and the regular pulse, the aspect ratio of the target in the picket case evolves differently than in the regular case and aspect ratios between 2 and 4 are obtained (Figure 4.18c).

Following experiments did not use the picket pulse, as no evidence of a greater drive efficiency was found, and the faster expansion of the targets implies a lower density, hence a lower optical depth, which makes the differences in line ratios harder to detect and characterise.

\subsection{Conclusions}

A new experimental platform for diagnosing the effects of the plasma geometry on the anisotropy of the emission of optically thick lines in HED plasmas was successfully developed at OMEGA. By using multiple target orientations and a carefully constructed laser drive configuration, self-consistent spatial and spectral data were produced from cylindrical targets with geometrical aspect ratios ranging from 0.4 to 1.4. These data enabled the characterization of the line anisotropies in the K-shell emission spectrum 
of mid-Z materials such as those used in Dot Spectroscopy experiments at NIF.

Strong evidence was found for enhancement and suppression of the optically thick line emission depending on the direction of observation. It was shown that twodimensional radiative transfer models can reproduce the observed data to a much higher degree than models which do not account for the plasma geometry. This allows for a correction in the temperatures and densities obtained from line ratios in HED or ICF experiments using dot-like plasmas as a spectral diagnostics.

The temperatures measured from the data are consistent among shots and for different views, which is in agreement with the plasma being uniform. The first experimental results of ion density calculations using exclusively the $\mathrm{He}-\alpha$ spectra of cylindrical HED plasmas [55] have also been obtained. These results are in good agreement with the measured density from the imaging data and show the capabilities of the method described in Section 3.4 as a spectroscopic diagnostic. This method has been extended with the explicit measurement of the plasma size, thus calculating the absolute opacity of the $y$ line. Several codes have been tested against the obtained data, showing disagreements in their atomic models of up to $50 \%$. 


\section{Chapter 5}

\section{Applications for ICF experiments}

This chapter is dedicated to the results from the Dot Spectroscopy experimental campaign at the $\mathrm{NIF}$, which was introduced in Section 1.1.2. Special focus is put on two shots from the 'warm subscale' experiments with Mn only dots. The cylindrical geometry of the dots is used as an additional diagnostic, by comparing the experimental results with those from the Cretin model described in Chapter 3 and benchmarked at the OMEGA experiments described in Chapter 4. This allows one to use the experimental line ratios as an optical depth diagnostic, and hence obtain an experimental value for the ion density of the plasma. This density calculation process does not follow the fully spectroscopic method described in Chapter 3, as in these experimental campaign, only the face-on emission spectrum was measured.

The chapter is structured as follows. A process to benchmark the absolute units of the spectral flux from the Cretin model is described in Section 5.1. Section 5.2 describes the diagnostic instruments that were fielded in this experiment, with focus on the NIF X-ray Spectrometer (NXS), used to obtain the manganese K-shell spectra. Although the experimental set-up was briefly described in Section 1.1.2, a complete and detailed description is given in Section 5.3. The raw results from the experiment are presented in Section 5.4 , with Section 5.5 dedicated to the spectroscopic analysis. 
In this section the process to obtain the ion density from the experimentally available data is detailed. The results obtained for the ion density are given in Section 5.6, where their significance is discussed by comparing the absolute flux predicted by the simulations with the experimental values. A comparison with the density values obtained by using a 1-dimensional spherical model is also presented in this section. Finally a summary of this work is given in Section 5.7 .

\subsection{Benchmarking the spectral flux units from the Cretin model}

The absolute spectral flux obtained from the cylindrical simulations used in this thesis was benchmarked against the results in Chambers et al. [77]. In their work, Chambers and coauthors studied the time-integrated K-shell emission of laser-generated aluminium plasmas. The targets were originally Al microdots $200 \mu \mathrm{m}$ wide and $1 \mu \mathrm{m}$ thick. The electron temperature of the generated plasma was measured using Thomson scattering, whereas the electron density was characterised using a combination of shadowgraphy and hydrodynamic simulations with the code MEDUSA [146, 147]. Their results for the electron temperature and density are shown in Figure 5.1 by the solid and dashed black lines respectively.

As the Cretin simulations assume that the plasma is in steady-state, and the spectrum from Chambers et al. is time-integrated, several points along the expansion have been simulated separately and time-integrated later. This, of course, is not ideal, as any time-dependent effects are lost, but it is sufficient for this purpose. The red squares and blue dots in Figure 5.1 correspond to the values of the temperature and density that were used as inputs of the Cretin simulations. The spacing between them is $\Delta t=100 \mathrm{ps}$, as this is the time resolution reported in the experiment.

For the other inputs in the Cretin model, the radius of the cylinder was assumed 


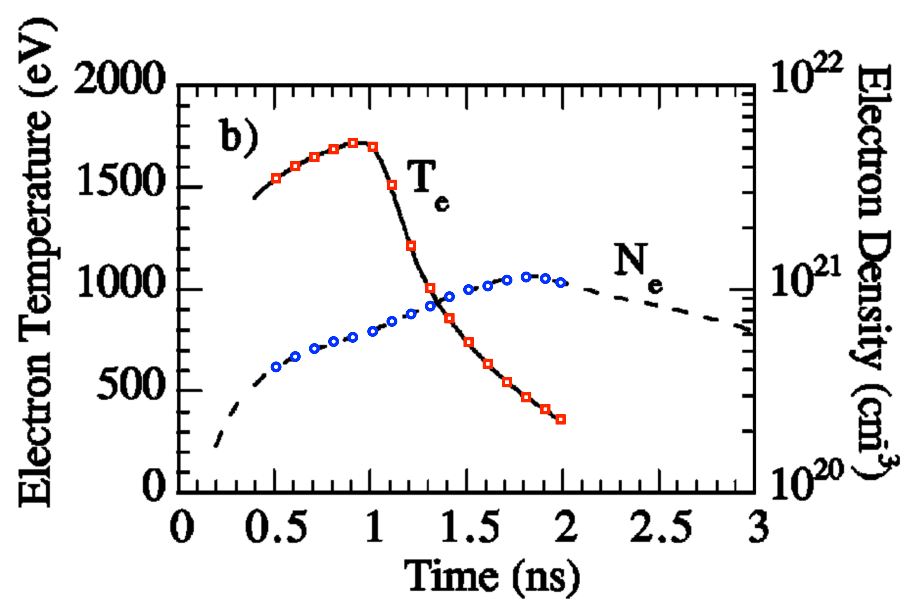

Figure 5.1: Electron temperatures (solid line) and electron densities (dashes) as a function of time at $300 \mu \mathrm{m}$ from the original target surface. Time 0 represents the start of the heater pulses. The red squares and blue circles correspond to the discrete values used in this work to obtain the synthetic spectrum.

to be constant and equal to the initial radius $(200 \mu \mathrm{m})$, whereas the thickness was obtained from the values of the electron density reported by Chambers et al., assuming conservation of particles. For each timestep, the thickness was adjusted such that the electron density obtained at the corresponding temperature agreed with the measured value. Given that in the experiment, the spectra were obtained at $90 \mathrm{deg}$ with respect to the normal of the microdot, the side-on spectra from the model were used to compare with the experimental results.

The synthetic spectra obtained for each timestep were then integrated and convolved with a Gaussian curve with a FWHM of $20 \mathrm{~m} \AA$ to account for the instrumental resolution in the experiment (as described in [77]). The raw result was also shifted $19 \mathrm{~m} \AA$ to greater wavelengths to reproduce the experimental line positions, and a constant background of $1.07 \times 10^{14}$ Photons $\mathrm{cm}^{-2} \mathrm{sr}^{-1} \mathrm{m \AA} \AA^{-1}$ was added.

The comparison between the experimental spectrum and the result from the Cretin simulations is shown in Figure 5.2. The solid black line correspond to the experimental result in absolute units, whereas the solid red line is the result from this work. The

\footnotetext{
${ }^{1}$ Reprinted figure with permission from [77. Copyright (2002) by the American Physical Society
} 


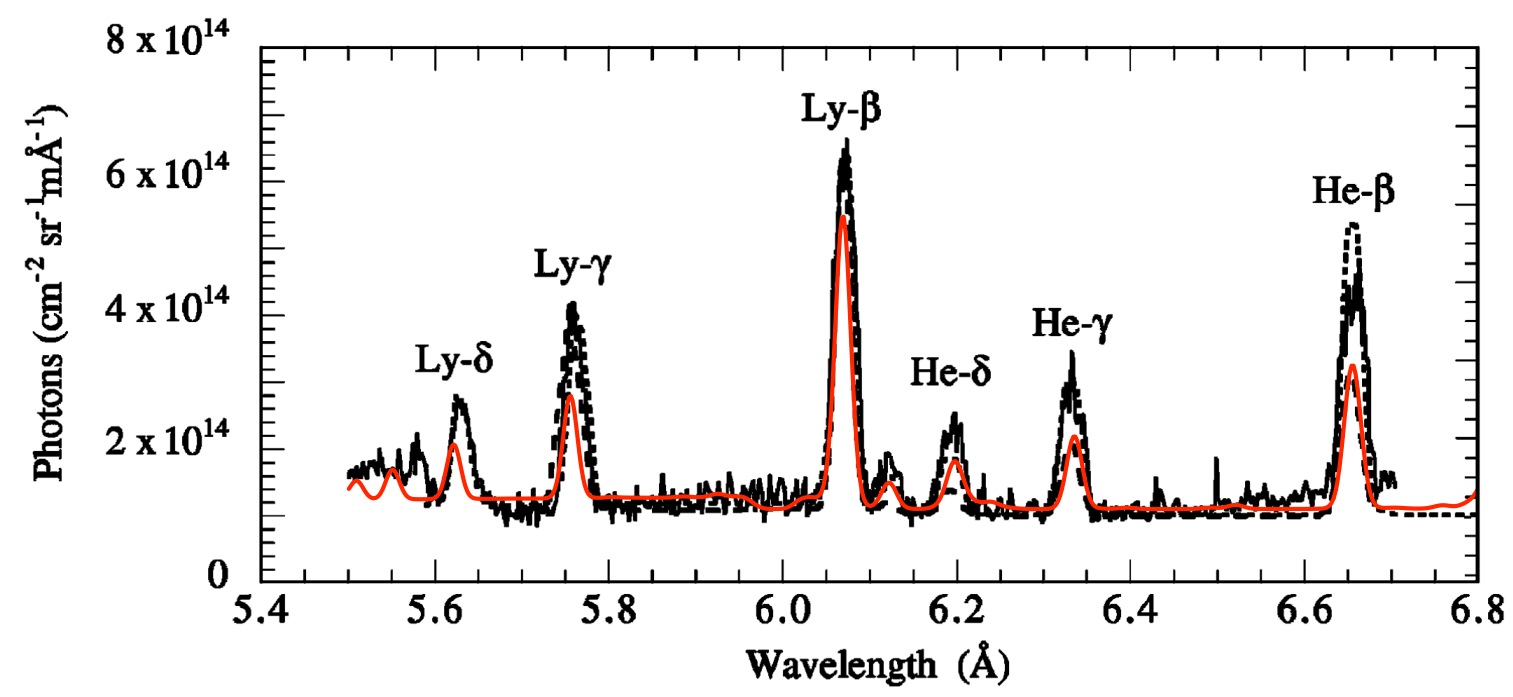

Figure 5.2: Experimental spectrum from [77] (solid black line) compared with the result from thie Cretin model (solid red line). The dashed and dotted black lines correspond to the steady-state and time-dependent simulations in [77] respectively. ${ }^{2}$

additional dashed and dotted black lines correspond respectively to the steady-state and time-dependent simulations done by the authors. It can be seen that the Cretin simulations agree with the data to a good extent. Furthermore, one can see in Figure 5.2 how the agreement is better between the Cretin simulations and the steadystate simulations done by the authors. This is expected, as in both cases the timedependent effects are not accounted for.

This agreement shows that the cylindrical Cretin model presented in this thesis can be used to characterise the absolute spectral flux emitted by HED plasmas.

\subsection{Diagnostic instruments}

In the Dot Spectroscopy experiments, two main diagnostics were used to obtain the data that are of interest to this work. The axial expansion of the dots, as well as their position along the axis the hohlraum were measured with an XRPINH, whose working principle and design were described in Section 4.1.2. In addition, the dots'

\footnotetext{
${ }^{2}$ See footnote 1
} 


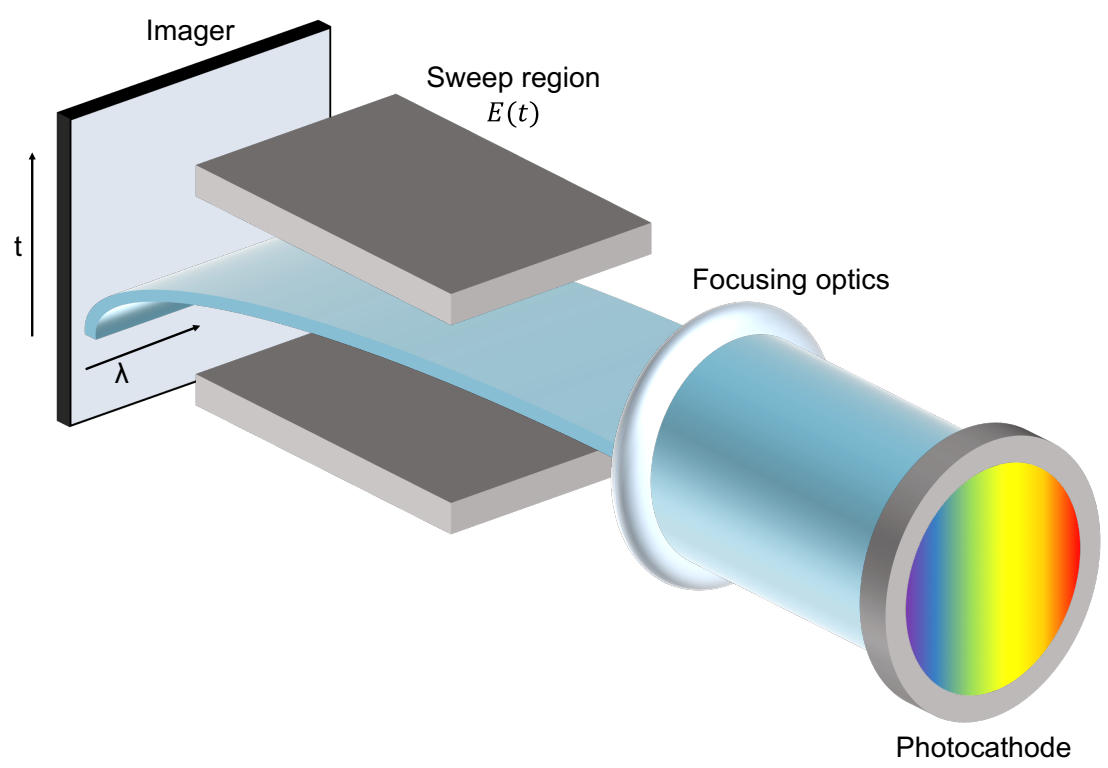

Figure 5.3: Schematic drawing of the design of a streak camera. The X-rays are impinge upon the photocathode generating a beam of electrons that gets focused into one dimension by the optics within the system. This beam is sent between through a sweeping electric field, and gets deflected before reaching the detector.

K-shell spectra were obtained with the NXS. This instrument uses a streak camera for time-resolving the emission, and is absolutely calibrated. In this section, the operating principle of streak cameras, as well as the overall design and operation of the NXS are described.

\subsubsection{Streak cameras}

Picosecond streak cameras [148, 149] are common diagnostics used in HED experiments to obtain information about the time evolution of a physical system, They are used for several different purposes as characterising short light pulses [150, 151], measuring the implosion velocity of ICF capsules [152, 153] or time-resolving the X-ray emission of plasmas [28, 29, 154] - purpose for which they were used in this case.

The working principle of a streak camera to time-resolve spectra is illustrated in Figure 5.3. The experimental spectrum (usually produced by diffracting off a Bragg crystal or similar) is shone upon a photocathode, which produces a beam of 
photoelectrons. The intensity of the electron beam varies across the dispersive axis, as a function of the spectral intensity. This electron beam is accelerated towards the detector applying a voltage gradient across the photocathode and possibly afterwards. Electrostatic lenses are then used to focus the beam across the non-dispersive axis. The resulting beam is sent through a sweeping electric field $E(t)$, which deflects the electrons in a direction perpendicular to the dispersive axis before they reach the imager. The sweeping electric field ensures that the position of the electron beam on the imager is a function of time. In the schematic shown in Figure 5.3, the vertical position of the electron beam in the imager uniquely determines the time at which the spectrum was produced.

The streak camera used in these experiments is the NIF diagnostic insertion manipulator imaging streak camera (DISC) [155, 156], which records the signal with an MCP coupled to a fibre bond CCD, in an equivalent way to the XRPINH mechanism described in Section 4.1.2. In particular, a CsI photocathode with a $250 \mu \mathrm{m}$ slit and a $10 \mathrm{~ns}$ sweep pulse was used, resulting in a temporal resolution of $\sim 80$ ps.

\subsubsection{The NIF X-ray Spectrometer}

The NXS is a time-resolved absolutely calibrated X-ray spectrometer designed for use at the NIF. Its operating principle is similar to that of the MSPEC described in Chapter 4, as an elliptical PET Bragg crystal is used to diffract the radiation that enters the spectrometer through the entrance aperture. However, the way that these spectra are recorded is different.

The spectra impinge upon two IPs, which obtain a time-integrated signal. These IPs are absolutely calibrated, in combination with the IP scanner at the NIF, so the time-integrated spectrum is obtained in absolute units [137, 138, 157]. In addition, the fact that two IPs are present further allows one to cross-check that there are no unexpected problems with one of the IPs. An example of the spectra recorded by 


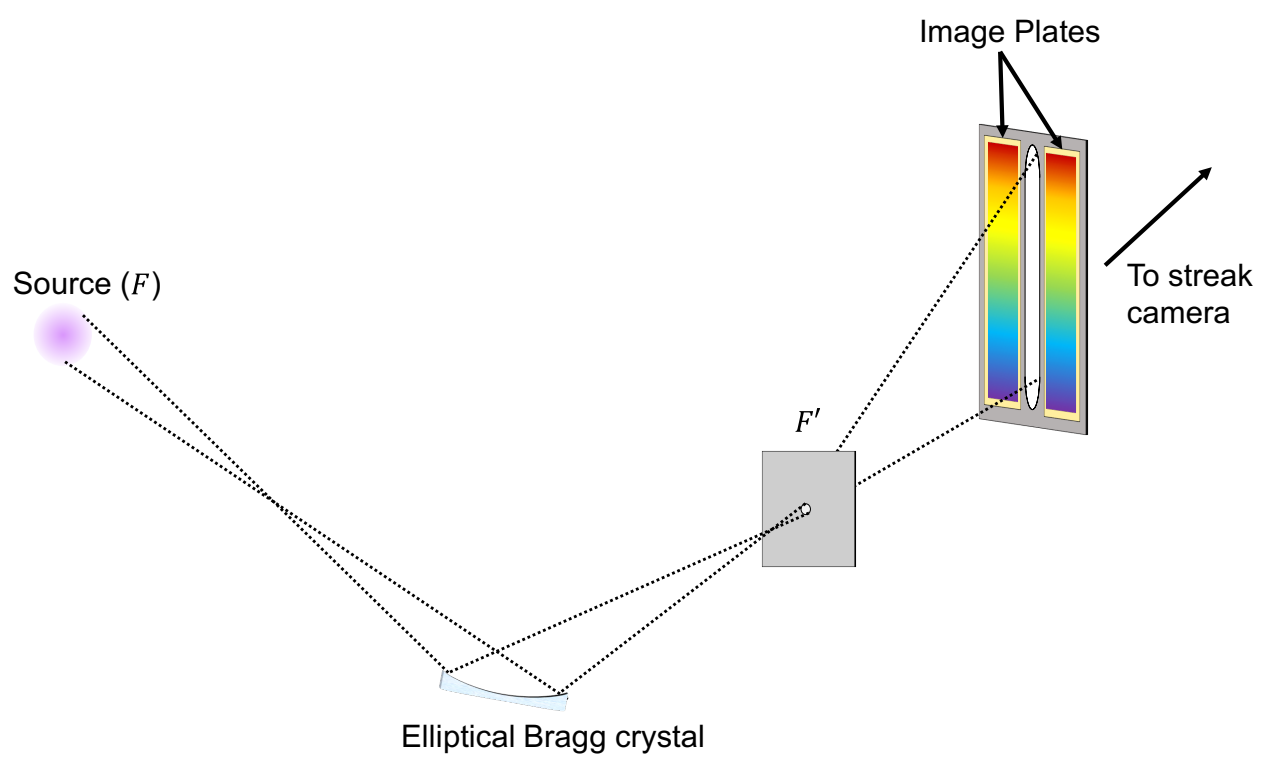

Figure 5.4: Schematic of the working principle of the NXS. Two images plate record time-integrated spectra that is then used to absolutely calibrate the units of the time-resolved spectrum obtained by the streak camera.

the IPs is shown in Figure 5.5. It can be seen in this picture that the bottom IP has two different levels of illumination across it. That is due to the fact that, to avoid overexposure of the $\mathrm{IP}$ and therefore saturation of the signal, different filters can be introduced in the NXS to cover diferent regions of the IP. By doing this, it is possible to extract the data from the region of the $\mathrm{IP}$ with the optimal exposure that does not produce saturation of the detector.

In the space between the two aforementioned IPs there is an aperture (which can also be seen in Figure 5.5 that leads to a streak camera which produces a timeresolved spectrum. This time-resolved signal is not calibrated per-se, but it is possible to convert the counts on the CCD to absolute units by integrating the CCD signal over time and comparing it with the absolute signal from the IPs. Therefore, the combination of the absolutely calibrated $\mathrm{IP}$ data and the streak camera returns a time-resolved spectrum in absolute units. 


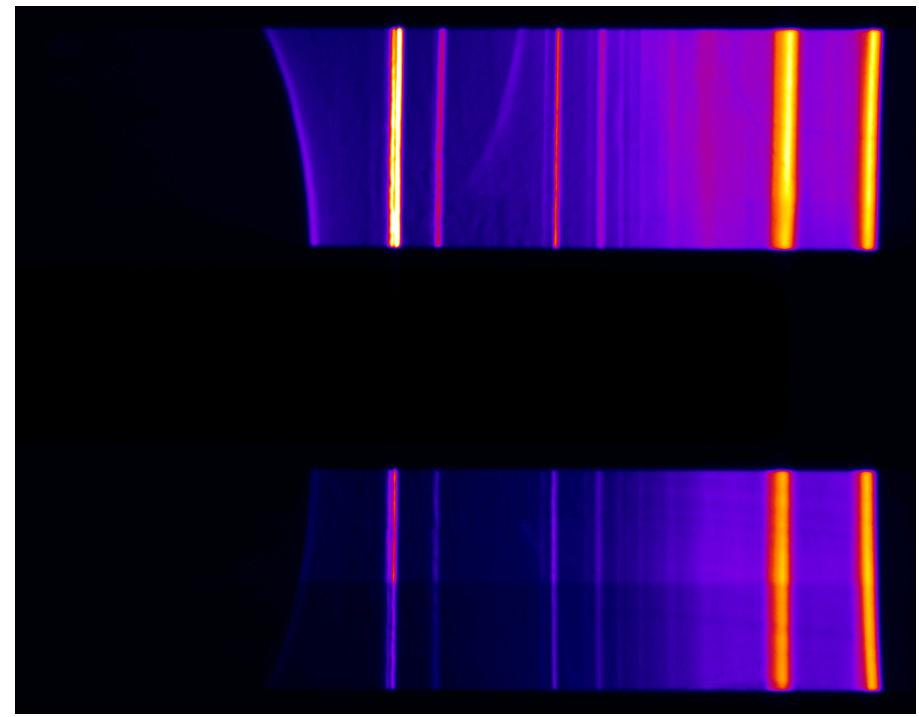

Figure 5.5: Example of spectra obtained by the IPs in the NXS. The region between both IPs is open so that the signal can reach the streak camera.

\subsection{Experimental set-up}

The experiments in the Dot Spectroscopy campaign were performed at the NIF laser, at the LLNL. This chapter is focused on the results from two particular shots in which the dots were pure manganese. The experimental conditions in both cases were nominally identical, with the exception of the thickness of the dots. In shot N150913-002, the thickness of the dot was $2400 \AA$, whereas in shot N151116-003, the thickness was $800 \AA$. This targets will hereafter be referred to as Target 2400 and Target 800 respectively. The radius of both dots was $400 \mu \mathrm{m}$.

In both cases, a $\mathrm{CH}$ capsule $25 \mu \mathrm{m}$ thick and $2.4 \mathrm{~mm}$ in diameter was mounted inside a ViewFactor target - a truncated hohlraum scaled to $80 \%$ the dimensions of a typical hohlraum used in ICF implosion experiments. The hohlraums were $4.69 \mathrm{~mm}$ in diameter and $5.92 \mathrm{~mm}$ long, with a Laser Entrance Hole (LEH) $3.1 \mathrm{~mm}$ in diameter in the non-truncated base. The capsule was centred $3.92 \mathrm{~mm}$ directly below the LEH and aligned with the axis of the hohlraum. Each dot was sputter-coated on top of a capsule keeping the axis of the dot aligned with the axis of the hohlraum. The hohlraums were filled with neopentane $\left(\mathrm{C}_{5} \mathrm{H}_{12}\right)$ at $1.37 \mathrm{mg} / \mathrm{cm}^{3}$ and positioned in the 


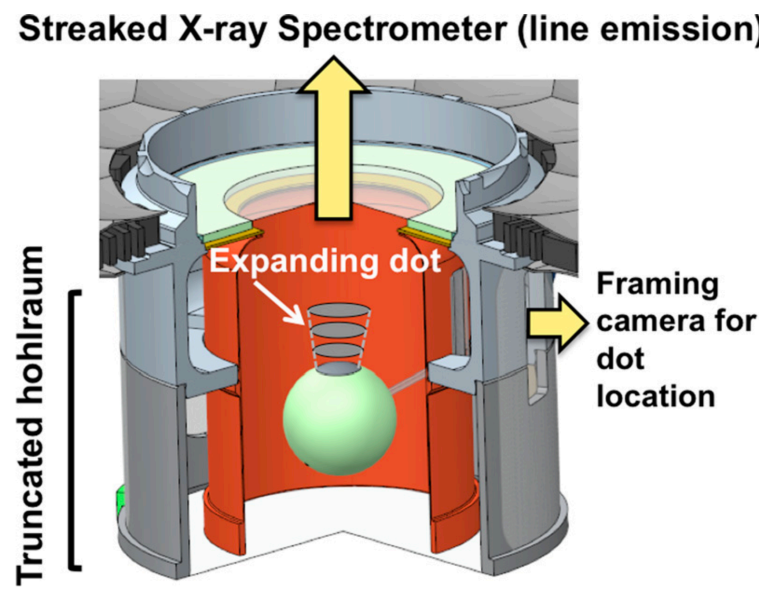

(a)

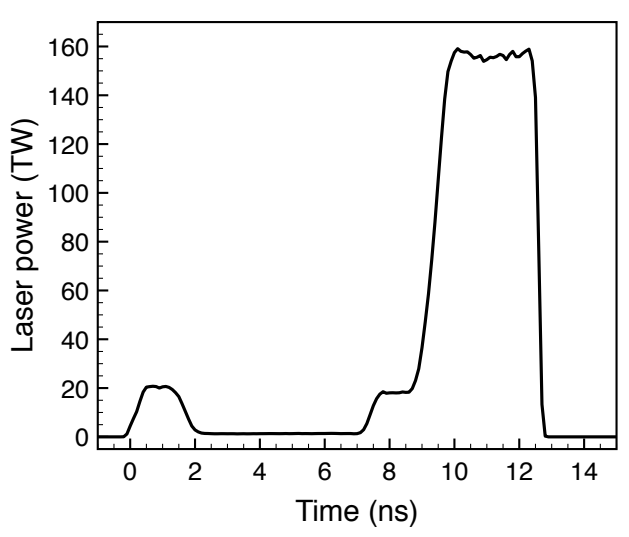

(b)

Figure 5.6: 5.6a Schematic of the experimental set-up showing the view of the different instruments and the position of the dot and the capsule inside the truncated hohlraum $\sqrt[3]{5.6 \mathrm{~b}}$ Shape of the laser pulse used in the experiment.

NIF target chamber such that the centre of the $\mathrm{CH}$ capsule was at the centre of the chamber. This set-up is shown in Figure 5.6a

While the K-shell emission of the dots was observed with the NXS looking down the hohlraum axis through the $\mathrm{LEH}$, the position and axial expansion of the dot were measured with an XRPINH. A rectangular aperture $(250 \mu \mathrm{m} \times 2300 \mu \mathrm{m})$ was cut in the hohlraum wall to provide a radial LOS, as shown in Figure 5.6a. This aperture was covered with a $\mathrm{HDC}$ window $80 \mu \mathrm{m}$ thick with dimensions $150 \mu \mathrm{m} \times 2200 \mu \mathrm{m}$, to avoid blockage of the aperture. The HDC window was held in place with $6 \mu \mathrm{m}$ thick Mylar strips. The $50 \mu \mathrm{m}$ gap to each side of the HDC window was also covered by the solid angle of the XRPINH, and therefore the thickness and position of the dot were measured through the $\mathrm{HDC}$ window and from both edges, to correct for any defects in the HDC transmission. An additional aperture was cut on the opposite wall (also covered with a HDC window) to minimise the signal from the gold wall on the XRPINH.

The laser profile used in the experiment was a $12.8 \mathrm{~ns}$ high-foot pulse, as shown

\footnotetext{
${ }^{3}$ Reprinted from [28] with the permission of AIP publishing.
} 


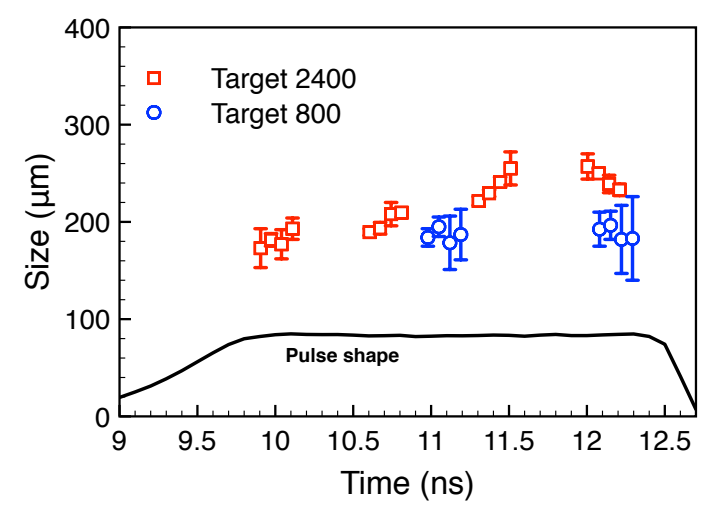

(a)

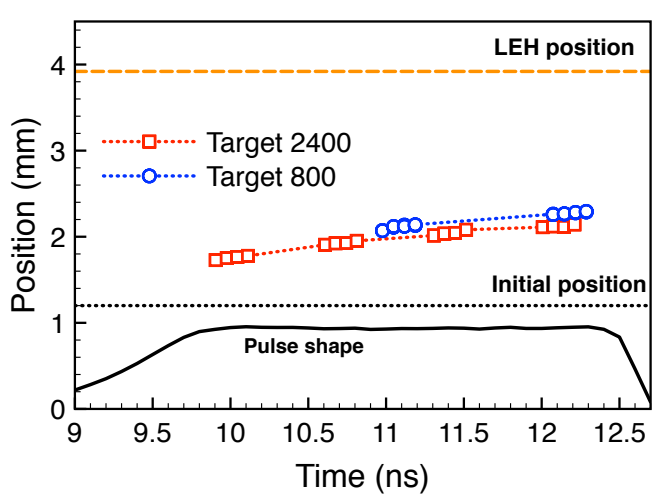

(b)

Figure 5.7: 5.7a) Thickness of both Mn dots measured through the HDC window as a function of time. (5.7b) Position of the dots across the hohlraum with respect to the LEH and their original position.

in Figure 5.6b, delivering $\sim 600 \mathrm{~kJ}$ of $3 \omega$ light energy in the hohlraum. Due to the truncated geometry of the hohlraum, not all the beams from NIF could be used, and therefore only 128 out of the 192 available were implemented. More details on the experimental set-up for this campaign can be found in Barrios et al. [28], where these experiments were first reported.

\subsection{Experimental results}

Similarly to the case in Chapter 4, the XRPINH data was used to diagnose the thickness of the targets along their expansion axis. This is shown in Figure 5.7a where the blue circles represent the Target 800 data, whereas the red squares correspond to Target 2400. This colour convention will be used for the remainder of this chapter. The error bars correspond to the differences in the thickness measured across the HDC window. For Target 800 there are half the values than for Target 2400, as in this case the frames in the XRPINH were activated in pairs, so twice the data points were taken at each timestep. The thickness of the dots does not change noticeably during the plateau of the laser pulse, although they have expanded axially by a factor 


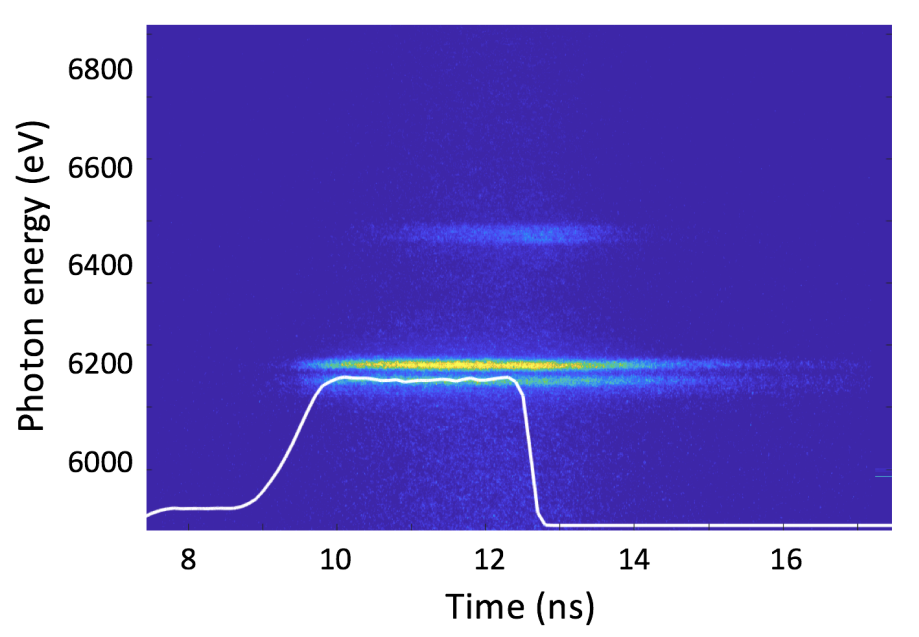

Figure 5.8: Streak-camera spectrum for Target 800. The laser pulse profile is shown in white. As the plasma evolves, it can be seen that the relative fluxes from the $\mathrm{He}-\alpha$ $(\sim 6200 \mathrm{eV})$ and $\mathrm{Ly}-\alpha(\sim 6500 \mathrm{eV})$ components change.

of $\sim 10^{3}$ in the first $9 \mathrm{~ns}$, considering their original thicknesses.

For the remainder of this chapter, the thickness of each dot is assumed to be constant during the plateau of the laser pulse, with values:

$$
\begin{aligned}
& h_{2400}=220 \pm 30 \mu \mathrm{m}, \\
& h_{800}=187 \pm 14 \mu \mathrm{m} .
\end{aligned}
$$

Note that both thicknesses are within 20\%, whereas the initial thicknesses of the dots differed by a factor of 3 .

The vertical position of the data on the XRPINH also gives information about the position of the dots inside the hohlraum as they move with the capsule. This information is crucial, as it allows one to know which part of the hohlraum is being diagnosed by the dots. Although in the two shots that are being analysed in this chapter the dots were coated on top of the capsule, there have been extensions to this campaign in which the dot was placed closer to the $\mathrm{LEH}$ or on the equator of the capsule to diagnose different regions of the hohlraum [29]. Figure 5.7b shows that, as expected in this case, both dots sit in similar positions inside the hohlraum, never reaching the regions close to the LEH. The error bars in this plot are contained within the size of the symbols. 
Good quality time-resolved spectral data were obtained for both targets. Figure 5.8 shows the streaked K-shell spectrum of Target 800 together with the laser pulse. The $\mathrm{x}$-axis shows the time with respect to the start of the pulse, whereas the $\mathrm{y}$ axis corresponds to the energy of the emitted photons. The main visible features correspond to the He- $\alpha$ complex $(\sim 6200 \mathrm{eV})$ and the Ly $-\alpha$ lines $(\sim 6500 \mathrm{eV})$. Note how during the rise of the pulse there is not any K-shell emission, as it starts when the pulse reaches a plateau. As the $\mathrm{Ly}-\alpha$ line is indicative of a high plasma temperature (it requires a higher ionisation state to be produced) its emission starts later in time than that of the He- $\alpha$ complex, as the plasma reaches a higher temperature. The inverse effect is observed after the laser pulse ends and the plasma starts to cool, with the Ly $-\alpha$ emission fading out first.

\subsection{Spectral analysis}

The spectra obtained with the NXS are continuously time-resolved, contrary to the case of the experiments described in Chapter 4, where they were grouped into frames. The temporal resolution of the streak camera is $\sim 80 \mathrm{ps}$, with $2.4 \mathrm{ps} /$ pixel, however, to minimise the signal-to-noise ratio, the spectra were integrated in windows of 130 pixels, equivalent to 312 ps.

The temperature distribution in the plasma was calculated at every timestep using a genetic algorithm [117] developed by Duane A. Liedahl. The process is identical to that described in Section 4.3 .2 and therefore it will not be discussed here. The obtained temperature distributions as a function of time for both shots are shown in Figure 5.9, where the central solid line corresponds to the centre of the distribution whereas the shaded area represents its width in both cases. The times shown in this figure correspond to the plateau of the laser pulse and therefore the laser pulse is not plotted. This applies to all the following figures. 


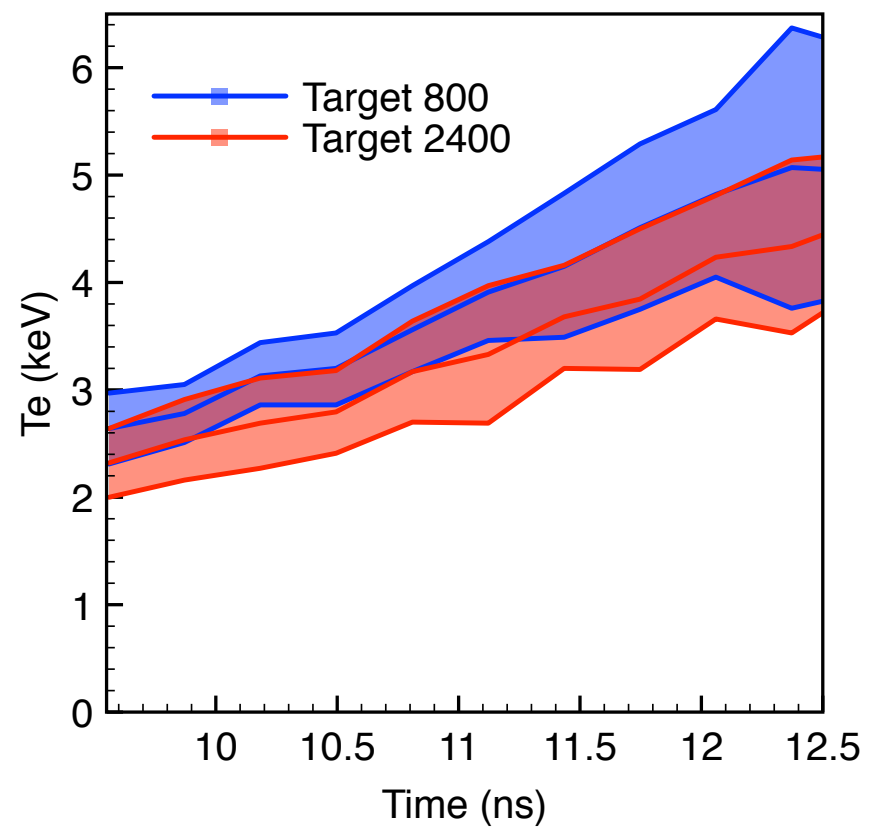

Figure 5.9: Temperature distributions as a function of time for both Target 800 and Target 2400. Both targets evolve with similar temperatures (within 20\%), despite their different initial thicknesses.

It is interesting to note that the temperature distributions of both targets agree within error bars for the whole duration of the laser pulse, and the central temperatures agree within $\sim 20 \%$. This is in agreement with the hypothesis that the dots' plasma equilibrates with its surroundings and reaches thermal equilibrium, and thus the temperature from the dot is indeed the temperature of the plasma at the corresponding region of the hohlraum.

Towards the end of the laser pulse, the width of the temperature distribution for Target 800 is $\sim 2.5 \mathrm{keV}$, which corresponds to $\sim 50 \%$ of its central value. For this reason for the rest of this chapter the latest timestep will not be accounted for, as the plasma can no longer be modeled to a good approximation by a uniform cylinder.

From the time-resolved spectra it is then possible to obtain a line-integrated $w / y$ ratio by fitting the spectra in the same way as described in Chapter 4 . These results are shown in Figure 5.10. It can be seen that Target 800 shows a consistently greater $w / y$ ratio than Target 2400 . This is not surprising per-se, as although both cylinders 


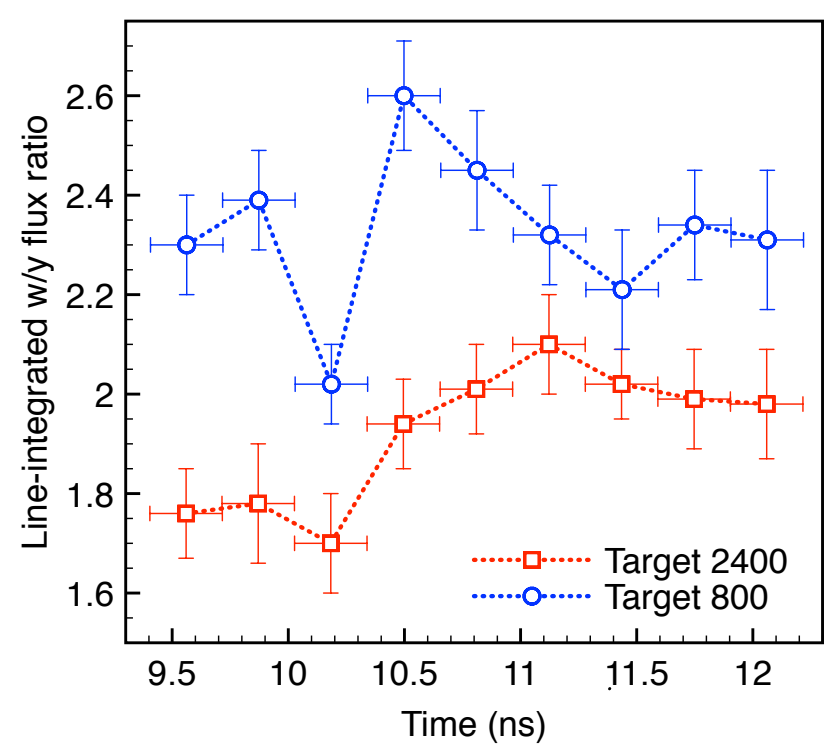

Figure 5.10: Results for the line-integrated $w / y$ flux ratio for both targets as a function of time. The ratio for Target 800 is consistently larger than for Target 2400, which is indicative of the dots having different optical depths. The horizontal error bars correspond to the width of the time-integrating window used to analyse the spectral data, whereas the vertical error bars correspond to the error in the line ratio obtained from the variance of the fitting parameters.

are at a similar temperature and have a similar thickness, they are intrinsically different plasmas, and their optical depth (on which the line ratios depend) is linked to their density and radius as well.

These quantities (radius and density) can be related to the known thickness of each plasma as

$$
R\left(n_{i}\right)=\sqrt{\frac{N}{\pi h n_{i}}}
$$

where $R$ is the plasma radius, $h$ is the corresponding thickness and $N$ is the total number of ions in the plasma. For each of the targets considered in this chapter $N$ is known and equal to

$$
N(i)=N_{A} \cdot \frac{\rho_{M n}}{M_{M n}} \pi H_{0}(i) R_{0}^{2},
$$

where $i$ is used to identify the targets (either Target 800 or Target 2400), $N_{A}$ is Avogrado's number, $\rho_{M n}$ and $M_{M n}$ are the solid density and atomic weight of manganese 


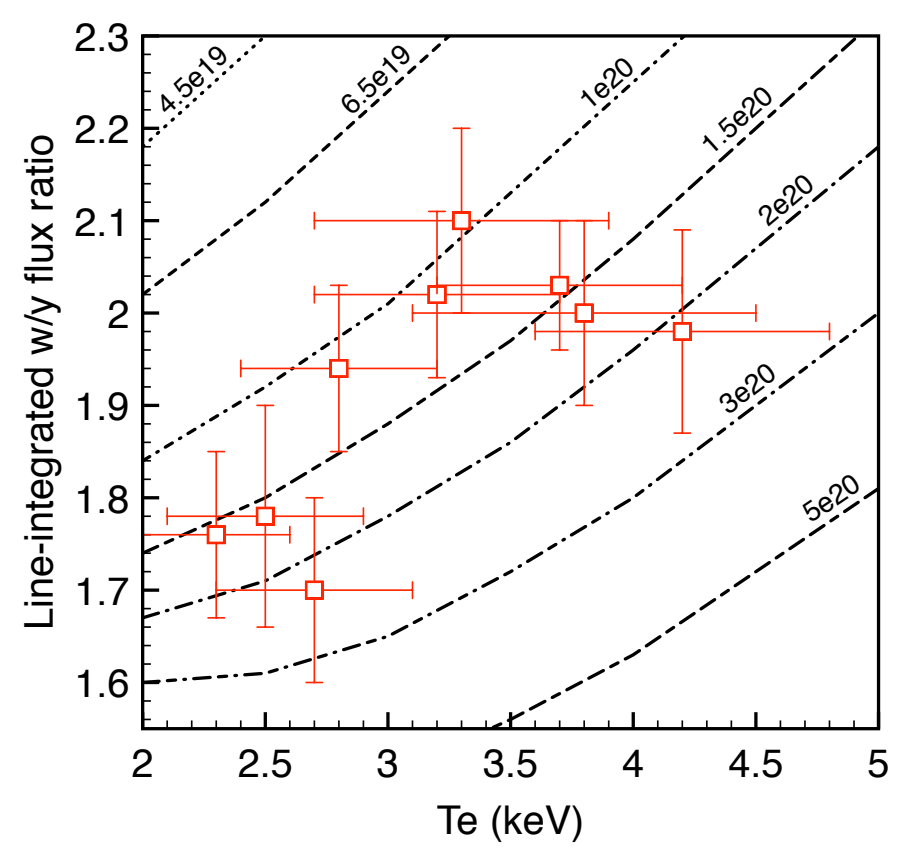

Figure 5.11: Map of the line-integrated $w / y$ flux ratio for Target 2400 as a function of the plasma temperature and ion density. The red squares correspond to the $w / y$ ratios from the experiment, plotted against temperature. The horizontal error bars correspond to the width of the temperature distribution, whereas the vertical lines represent the error in the line ratios. Each black line corresponds to the line ratios from the Cretin model for a given ion density as a function of temperature. The lines are labelled with the corresponding ion density for clarity.

respectively, and $H_{0}(i)$ and $R_{0}$ are the initial thickness and radius of each target. Assuming that the particles in the dot are conserved, this number will remain constant through the expansion in both cases.

Using the values of $h$ measured from the XRPINH data for each target and the relation between the radius of a cylinder and the ion density (from Equation 5.1), it is possible to generate cylindrical simulations using the Cretin model for a grid of fixed temperatures and ion densities. This grid of simulations produces a 'map' of the expected $w / y$ ratio for each of the targets (assuming perfectly cylindrical geometry) as a function of their temperature and ion density. As the experimental $w / y$ ratios and temperatures are known, it is now possible to compare the experimental results with the results from the cylindrical simulations. Figure 5.11 shows the 'map' of the $w / y$ ratio as a function of temperature and ion density for Target 2400 (for Target 
800 , a different map is obtained as the values of $N$ and $h$ are different). The $\mathrm{x}$-axis corresponds to temperature, whereas the $y$ axis shows the $w / y$ ratio. Each of the black lines across the plot corresponds to a single and fixed ion density. The red squares represent the experimental data with the corresponding uncertainty regions. Note that time is not explicitly plotted in this figure, but given that the temperature increases monotonically during the plateau of the pulse (as shown in Figure 5.9), moving up in temperature is equivalent to moving later in time.

\subsection{Results for the plasma density}

From the map shown in Figure 5.11 (and the equivalent result for Target 800), a value of $n_{i}$ with an associated uncertainty can be interpolated for each of the experimental $\left(T_{e}, w / y\right)$ points. These are shown in Figure 5.12 together with the predictions for a $3200 \AA$ thick dot, from the 2-dimensional hydrodynamic simulations run prior to the experiment, obtained with the code HYDRA [57]. It can be seen that the experimental results for the ion density of the dots are in agreement with the hydrodynamic simulations by a factor of $<2$ within the error bars.

Furthermore, after $t=11 \mathrm{~ns}$, both targets have the same density within error bars. This is particularly interesting, because it is from that moment on that the thickness of Target 800 is known whereas the results obtained for the density of Target 800 before $11 \mathrm{~ns}$ rely on the assumption that the dot is not expanding. For the times on which the thickness of both targets is known, both of them have the same density. This is in agreement with both dots reaching equilibrium with the surrounding plasma regardless of their initial conditions, and therefore being a good diagnostic of the conditions of the plasma inside the hohlraum.

For earlier times, there is a clear difference between the ion density values obtained for both shots (up to a factor of 3). Although this might be an actual physical effect, 


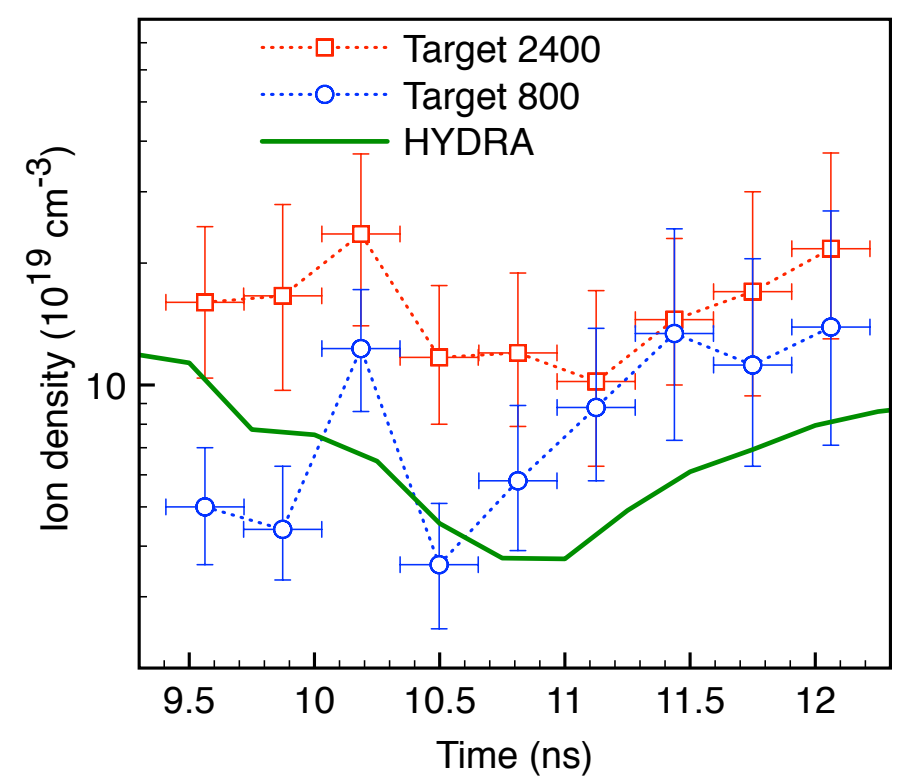

Figure 5.12: Ion density of both targets obtained from their $w / y$ ratio, assuming a cylindrical shape, compared with the predictions from HYDRA. The error bars come from the width of the temperature distributions, as well as the error in the determination of the $w / y$ ratio.

as the dots are initially different, there is another possibility that needs consideration. During this analysis, it has been assumed that neither of the targets expanded axially during the plateau of the laser pulse, based on the XRPINH results for Target 2400, whose thickness changed by $\pm 14 \%$ during the exposure time. However, it is possible that Target 800 is still expanding during those early times. If that were the case, and the thickness of Target 800 at early times were smaller than what has been assumed, a given ion density would correspond to a smaller aspect ratio (smaller $h$ and larger $R$ to keep to volume constant), hence to a larger $w / y$ ratio. By looking at Figure 5.11, it can be seen that, in that case, the constant-density lines would be shifted upwards for the first timesteps in the case of Target 800, which would result in a higher density begin inferred from the experimental data, reducing the differences between both dots.

Nevertheless, with the information available from these shots, it is not possible to confirm or rule out this possibility and more work needs to be done to check whether 


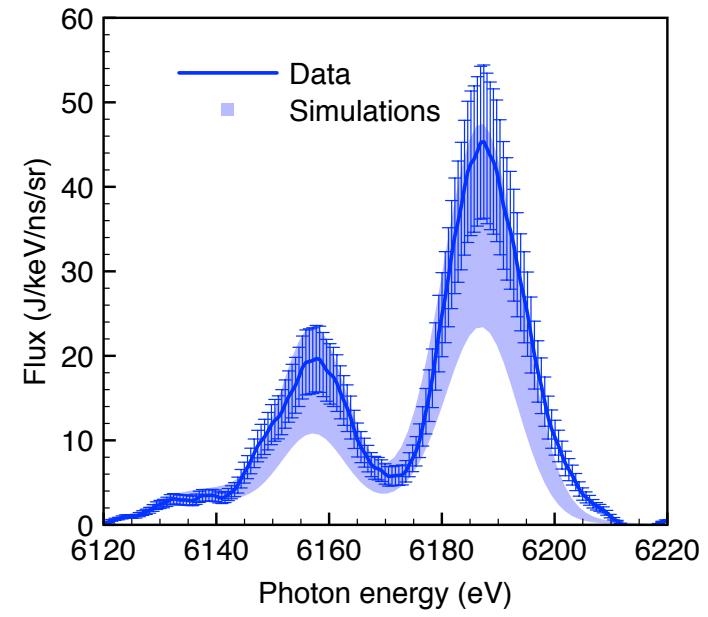

(a)

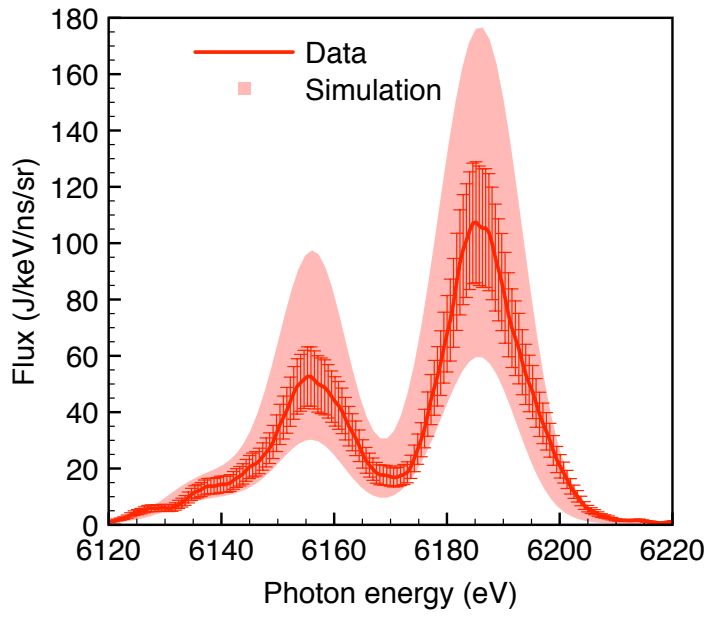

(b)

Figure 5.13: Comparison between the absolute flux at $t=11.1 \mathrm{~ns}$ measured in the experiment (solid lines) and the result from the cylindrical model for the obtained temperatures and densities (shaded regions) for Target 800 (5.13a) and Target 2400 (5.13b). A $20 \%$ error bar has been included in the experimental data to account for the uncertainty in the NXS calibration. The width of the shaded regions shows the possible values of the absolute flux predicted by the simulations within the uncertainty in the temperature and density measurements.

the dots are reaching equilibrium with the surrounding plasma during the early times.

\subsubsection{Absolute flux}

Although the density results obtained in the previous section are in reasonable agreement with the hydrodynamic simulations, it is still necessary to check whether they are consistent with other experimental data. This is possible in this particular platform, as the data from the $\mathrm{NXS}$ are absolutely calibrated, and so the experimental spectra are measured in units of $\mathrm{J} / \mathrm{keV} / \mathrm{ns} / \mathrm{sr}$. In addition, the Cretin simulations return the spectra measured at the detector in absolute units as well and therefore, as shown in Section 5.1, it is possible to compare the measured flux in absolute units with the results from the Cretin simulations at the measured ion densities.

Figure 5.13 shows one example of this result for each target. The spectra shown in this figure were taken at $t=11.1 \mathrm{~ns}$. The solid lines correspond to the experimental 


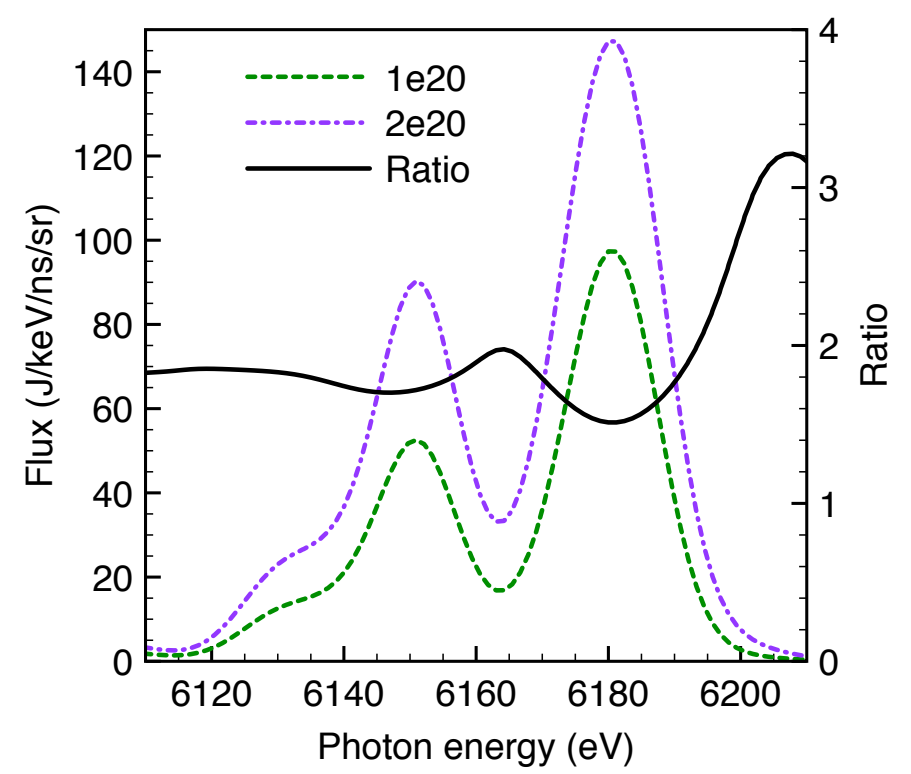

Figure 5.14: Spectral flux from the Cretin simulations for a $3 \mathrm{keV}$ plasma for two different ion densities differing by a factor of two. The ratio between them is shown as the solid black line.

values of the spectral flux, where a $20 \%$ error bar has been included to account for the uncertainty in the absolute calibration. The shaded areas correspond to the absolute flux predicted by the simulations. The extent of this area arises from the uncertainty in the measured temperature and density, as the greater the values of these parameters, the greater the flux obtained from the Cretin model.

It can be seen that the results from the computational model agree with the experimental spectra well within error bars. This is particularly significant because the absolute flux obtained from the simulations is proportional to the ion density (as the number of total ions is fixed). This is shown in Figure 5.14, where the spectral fluxes from two simulations are compared. Both simulations have the same temperature $(3 \mathrm{keV})$ but their ion densities differ by a factor of $2\left(10^{20} \mathrm{~cm}^{-3}\right.$ and $2 \times 10^{20} \mathrm{~cm}^{-3}$ respectively). The ratio between both fluxes is shown as the black line. It can be seen that this ratio has a roughly constant value of $\sim 2$ in the regions where the lines lie, following the expected proportionality with the ion density.

This result shows that the ion densities obtained for both targets by comparing 


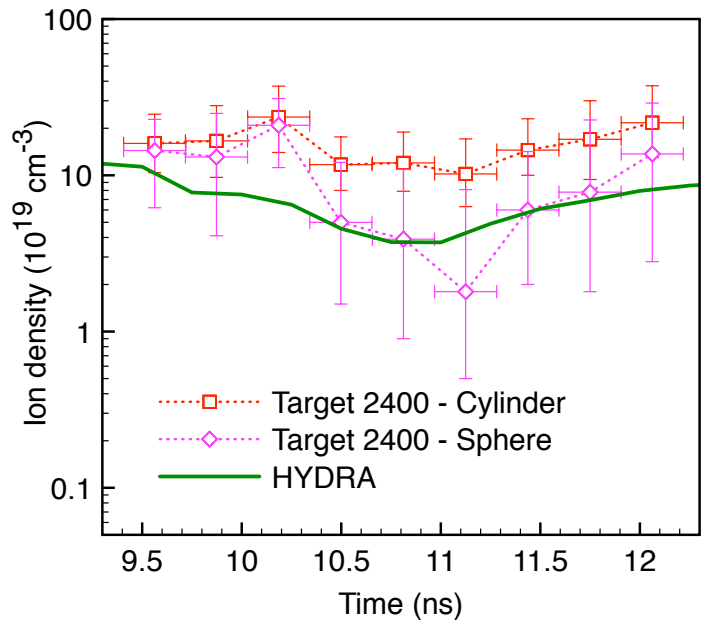

(a)

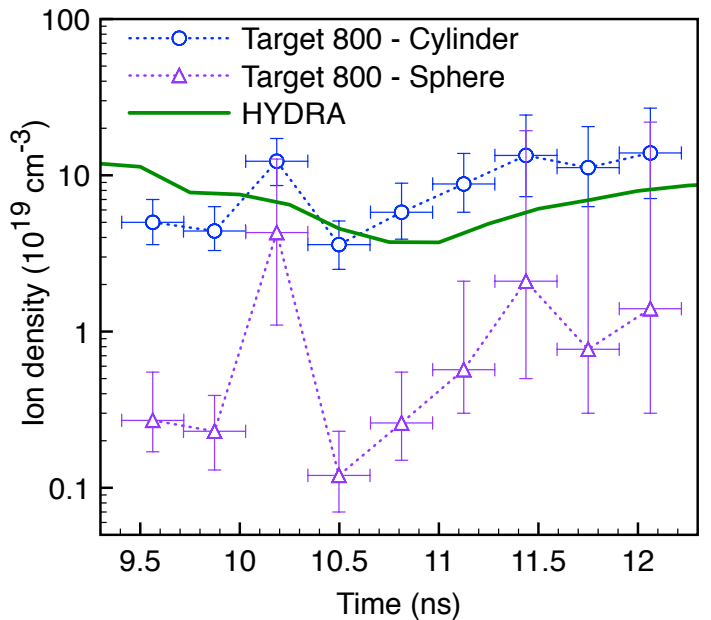

(b)

Figure 5.15: Results for the ion density of the plasma obtained using the spherical model for Target 2400 (5.15a) and Target 800 (5.15b), compared with the corresponding results from the cylindrical model and the HYDRA simulations.

their $w / y$ ratios with the results from the cylindrical model are consistent with the experimental data for the absolute flux, proving that the plasma geometry can be used as an additional spectroscopic diagnostic.

\subsubsection{Results for spherical model}

So far, it has been shown how the temperature and $w / y$ ratios extracted from the experimental data can be coupled with the cylindrical model that was benchmarked at the OMEGA experiments, to obtain a measurement of the ion density of the plasma. However, it is still unclear how important it is to treat the plasma as a cylinder, and not as an isotropic system. In Figure 4.14 it was shown how for the experimental conditions achieved at OMEGA, the obtained $w / y$ ratios for face-on view of a cylinder were similar to those obtained for a sphere. For this reason, one might ask what the obtained density would be if a sphere were assumed instead of a cylinder in this case.

To answer this question, the exact same procedure was followed using a spherical model instead. In this case, for each value of $n_{i}$ in the grid, the radius of the sphere 
was calculated as

$$
R\left(n_{i}\right)=\left(\frac{N}{\frac{4}{3} \pi n_{i}}\right)^{1 / 3},
$$

to force conservation of particles. A $w / y$ map equivalent to that in Figure 5.11 can then be constructed for each target, thus obtaining the equivalent values of the ion density. Figure 5.15 shows the results for the ion density obtained for both targets when assuming a spherical plasma. The cylindrical equivalent and the hydrodynamic predictions are also shown for comparison and the scale of the y-axis has been increased to show all the data.

Note how, although for Target 2400 the spherical predictions are in agreement with the results from the cylindrical model (within error bars), in the case of Target 800 , there is a difference of one order of magnitude between the results for both models. Given that Figure 5.13 shows that the cylindrical simulations give the right density values for both targets, this difference implies that the spherical model does not reproduce the experimental data and is not a reliable way to obtain the ion density of the plasma.

\subsubsection{Discrepancies with hydrodynamic predictions}

In the previous section, it has been claimed that there is good agreement between the ion density values obtained from the cylindrical model and the hydrodynamic predictions. However, although the values agree by a factor of $<2$ within error bars, it can be seen from Figure 5.12 that the predictions from HYDRA are consistently lower than the values presented in this work (from $t=11 \mathrm{~ns}$ on).

One cause that might contribute to this discrepancy comes from the fact that the dot's plasma is not really a perfect cylinder. Note how the results from the spherical model, shown in the previous section, predict a lower density than obtained with the perfect cylinder case. 


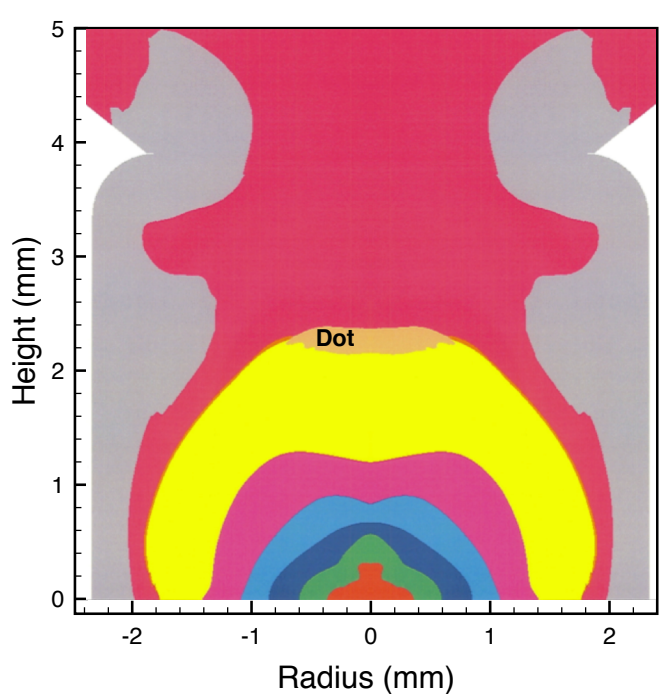

Figure 5.16: Axial section from the HYDRA simulations, showing the predicted dot's position and shape for a $3200 \AA$ thick dot at $t=11.5 \mathrm{~ns}$. The spatial coordinates are measured from the centre of the capsule. Each colour corresponds to a different material, as mixing is not permitted in these simulations. The dot's plasma is plotted in light orange, as indicated in the figure. It can be seen that the predicted shape of the dot is not a perfect cylinder, as the thickness decreases towards the edges.

The shape of the cylinder was not explicitly measured in the experiment, but it is possible to get an estimate of the dot's shape from the hydrodynamic simulations. An example is shown in Figure 5.16, where the result for a $3200 \AA$ dot at $t=11 \mathrm{~ns}$ is plotted. The shape of the dot seems to be more squeezed towards the edges of the cylinder with a slight dip in the centre, resulting in a more plate-like shape, as seen. These deviations from a perfect cylinder, as shown in Section 5.6.2, modify the $w / y$ ratio observed from any view, and in particular for the face-on view, as in this case. This modifies the density map shown in Figure 5.11, and its equivalent for Target 800, thus modifying the density results presented in this chapter.

\subsection{Conclusions}

In this chapter, the Dot Spectroscopy experiments at NIF (specifically, those in the warm subscale) have been described, detailing the experimental set-up and different 


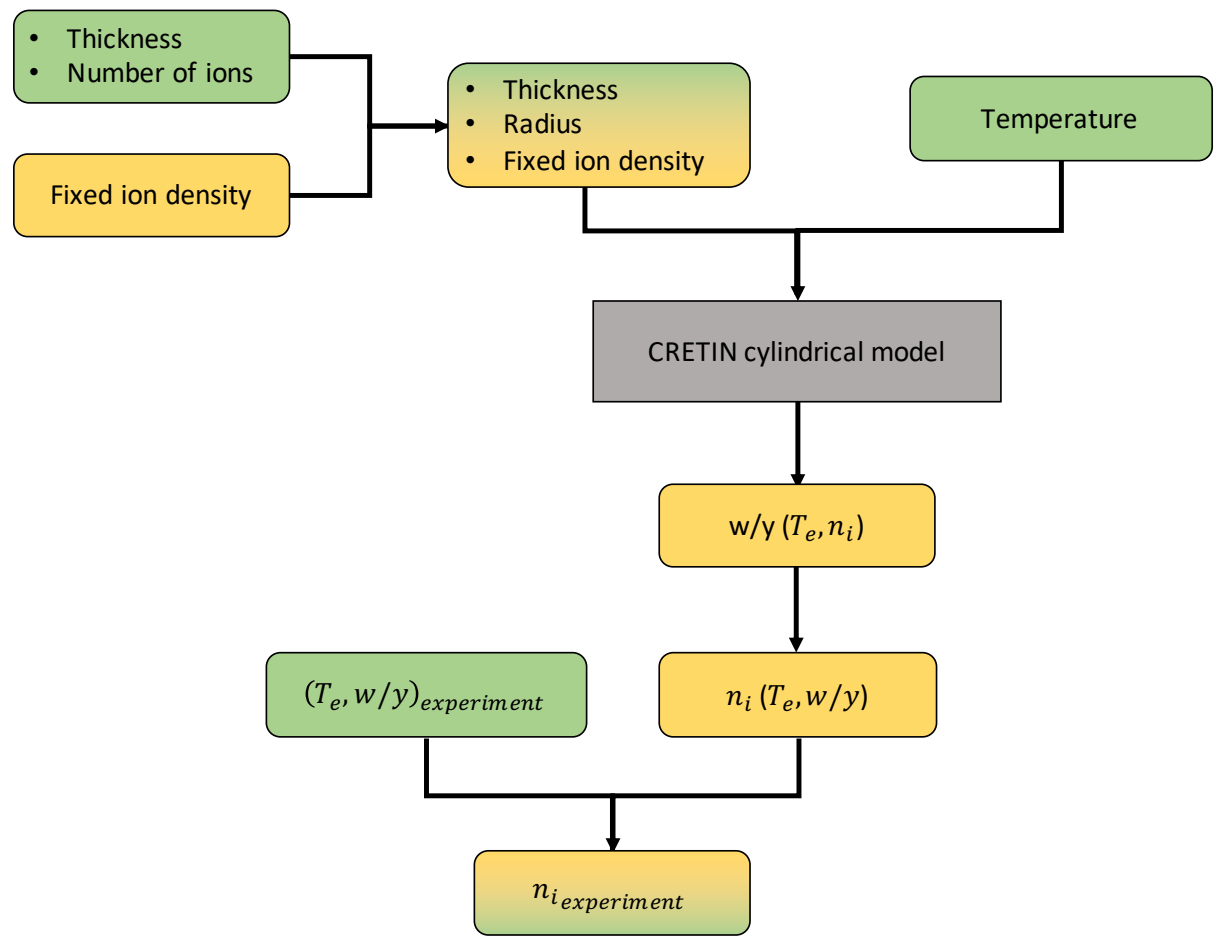

Figure 5.17: Schematic diagram of the process described in this chapter to measure the plasma density from the $w / y$ ratio. The green parameters represent data that were obtained from the experiment whereas those in yellow come from simulations.

instruments used for diagnosis. Two particular shots have been analysed, namely N150913-002 and N151116-003 (Target 2400 and Target 800 respectively). The conditions for both of these shots were identical, only differing on the thickness of the dots used, making them ideal candidates to study the effects of the geometry on the plasma spectrum using the tools developed throughout this thesis.

By using a genetic algorithm approach, it was found that both dots reach similar temperatures (within 20\%), and have a similar distribution during the plateau of the laser pulse. This supports the idea of the dots' plasma reaching thermal equilibrium with the surrounding plasma, and therefore it being a reliable temperature diagnostic.

Combining the predictions for the $w / y$ ratio from the Cretin model benchmarked in Chapter 4 with the temperature obtained from the time-resolved spectra and additional information about the thickness of the dots throughout their expansion, it was possible to obtain the values of the ion density such that a perfect plasma cylinder 
in the experimental conditions would have the same $w / y$ ratio as the data. As this process is slightly convoluted, Figure 5.17 shows an schematic diagram of it. The green boxes correspond to experimentally obtained parameters, whereas the yellow ones come from the simulations.

The values of the ion density thereby obtained agree with the hydrodynamic predictions for a $3200 \AA$ thick dot, up to a factor of $\sim 2$ within error bars. Moreover, both dots present the same ion density values after $t=11 \mathrm{~ns}$, moment from which the thickness of Target 800 is experimentally measured. This agreement between both dots and with the simulations for an even thicker target, is consistent with the dots reaching equilibrium with the surrounding plasma, so the density does not depend on the dot itself. Further work needs to be done to ensure this, but it would indicate that the dot can not only be used to map the plasma temperature in the hohlraum, but the density too.

By benchmarking the absolute units of the spectral flux obtained from the simulations with the results from Chambers et al. [77], it was possible to prove that the flux predicted by the Cretin model at the measured densities agrees within uncertainty bars with the experimental measurements obtained with the NXS. As the spectral flux is proportional to the ion density, this agreement of the absolute units provides further confidence on the validity of the density values obtained with this method.

It was also shown that if the same process was followed using a spherical model and not taking into account the fact that the plasma is cylindrical, the results obtained for the ion density would be different between both dots. In particular for Target 800, the density obtained assuming a spherical plasma is an order of magnitude lower than that obtained with the cylindrical model, yielding a disagreement between the experimental and simulated spectral fluxes. This implies that the spherical model is not reliable in a general case, as it overlooks the spectral changes due to the plasma geometry. 
The work presented in this chapter shows how the geometry of the plasma can be used as an additional spectroscopic diagnostic, providing useful information about the optical depth and hence the density of the plasma. These are the first results of time-resolved coupled temperature and density measurements inside a hohlraum during an ICF experiment, which shows the importance and usefulness of this work in characterising the effects of the plasma geometry in the X-ray spectrum of HED plasmas. 


\section{Chapter 6}

\section{Conclusions and future work}

\subsection{Summary and conclusions}

This thesis has presented an analysis of the effects of the plasma geometry in their X-ray emission spectra in the HED regime. This was done with an analysis of the underlying theory, together with multidimensional simulations, which were later benchmarked by experiments at the OMEGA laser facility. Finally, these results allowed for a better analysis of the data from the Dot Specroscopy experiments at NIF, showing that these geometric effects can be used as an additional diagnostic method, instead of them being an experimental drawback.

In Chapter 2, a discussion of the physical reasons why the X-ray emission is modified by the plasma geometry was presented. It was shown how the difference between the mean chord of a plasma and the LOS through which the spectrum is detected affects the intensity of the optically thick components of the spectrum. If the LOS of the escape path is shorter than the mean chord, the attenuation of the emission due to absorption of radiation does not overcome the photopumping effect caused by the radiation field within the plasma. Therefore the optically thick emission is enhanced with respect to the optically thin case. The opposite happens if the LOS 
is greater than the mean chord, with the intensity of the optically thick emission being reduced with respect to the case with no absorption within the plasma.

A detailed mathematical analysis of radiative transfer in several different geometries was done, finding that the planar slab or the cylindrical geometry were best to study these effects experimentally. The reason for this preference is that in said geometries, the two characteristic dimensions of the plasma (thickness and width) are not linked by any intrinsic symmetry of the system, whereas for the other cases of study, namely the hemi-spherical or hemi-ellipsoidal hollow shell and the torus, there are further constraints on the geometry limiting the scope of the geometric variations that can be present. As cylinders are the simplest form of a planar slab, due to their symmetry around the axis, and cylindrical targets are easy to manufacture for HED experiments, focus was put on this geometry for the rest of the thesis.

Chapter 3 focuses on analysing cylindrical plasmas from a experimental perspective, focusing on the He- $\alpha$ complex of the spectrum $\left(1 s 2 l \rightarrow 1 s^{2}\right)$, and in particular on the $w\left(1 s 2 p{ }^{1} P_{1} \rightarrow 1 s^{2}{ }^{1} S_{0}\right)$ and $y\left(1 s 2 p{ }^{3} P_{1} \rightarrow 1 s^{2}{ }^{1} S_{0}\right)$ lines. These lines are the main components of the $\mathrm{He}-\alpha$ complex in $\mathrm{HED}$ conditions, and are two of the brightest lines in a typical K-shell spectrum at the densities and temperatures characteristic of these systems. They are of particular interest for this work, as the opacity of the $w$ line is several times greater than that of the $y$ line, which allows one to design experiments that render the $w$ line optically thick, while keeping the $y$ line optically thin. In this case, their ratio can be used as a diagnostic of the effects of the plasma geometry.

In a real experiment, instrumental broadening and noise make good measurements of the frequency-resolved spectral flux from a plasma difficult to obtain. The lineintegrated flux is however, a starker measurement, as the instrumental broadening affects all the lines similarly, and the lineshapes are usually Gaussian, Lorentzian or a combination of both (Voigt-like). This allows one to fit the data to a known function, 
minimising the effect of the noise. For this reason, the line-integrated $w / y$ ratio is the main quantity used in this thesis to characterise the geometric effects.

For the particular case of a plasma cylinder there are two preferential directions of observation, namely face-on or down the axis, and side-on or in the radial direction. In Section 3.4 it was shown how the quotient between the line-integrated $w / y$ ratio for both of these views depends only on the optical depth at the centre of the $y$ line and the aspect ratio of the cylinder $(H / R)$, as given by Equations 3.11 and 3.12 . Furthermore, given that the optical depth of any transition in a plasma is directly proportional to its ion density, from these quotient of ratios it is possible to obtain an exact value of the ion density of the plasma. In addition, using a radiative transfer model built for this work using the code Cretin [31, small deviations from a perfect plasma cylinder were studied in Section 3.4.4, by considering the case of a frustum. Figure $3.8 \mathrm{~b}$ shows how these deviations modify the results obtained for the ion density. It can be seen that agreement within a factor of $\sim 2$ is still reached for the range of deviations considered here.

To experimentally validate these results and the computational model, the 'OpticalDepth' experiments were performed at the OMEGA laser facility. They are described in detail in Chapter 4. In said experiments, a Fe:V dot was buried in a cylindrical Be tamper, which was irradiated by the $3 \omega$ component of the laser. In this way, a Fe: $\mathrm{V}$ plasma cylinder is created, and its expansion is confined mostly to the axial direction. The face-on and side-on spectra of the cylinder were measured at four different timesteps using two perpendicular MSPEC coupled to framing cameras, whereas the radial and axial expansion were measured at the same times using two perpendicular XRPINH cameras. To minimise any instrumental deffects modifying the obtained results, two different target orientations were used, with one orientation of the targets being rotated $90^{\circ}$ with respect to the other, so all MSPEC and XRPINH obtained both a face-on and side-on view in subsequent shots. 
The face-on and side-on $w / y$ ratios obtained from this campaign provided an experimental validation of the ion density calculation method described above. In addition, as shown in Figure 4.14, they were used to benchmark the line ratios obtained from the Cretin model. It was found that including the cylindrical geometry in the simulations, the experimental line ratios could be reproduced with a much better agreement than the 1-dimensional case of a spherical plasma, in which the enhancement or supression of the optically thick lines as a function of the different LOS are not considered.

Experimentally benchmarking the $w / y$ results from the Cretin model, provides a useful tool to analyse $\mathrm{HED}$ and $\mathrm{ICF}$ experiments involving cylindrical plasmas. In particular, Chapter 5 focuses on the Dot Spectroscopy experiments at NIF, where the K-shell emission spectrum of a Mn dot was used to diagnose the electron temperature inside an $\mathrm{ICF}$ hohlraum. By comparing the experimental $w / y$ ratio with the predictions from the model as a function of temperature and density, it was possible to obtain an estimate of the experimental ion density as a function of time, as shown in Figure 5.12. This information was previously unavailable, and agrees with the hydrodynamic predictions by a factor of 2 within error bars. Furthermore, it was shown that if a spherical geometry is assumed, neglecting the cylindrical symmetry of the dots, the predictions for the ion density differ by up to an order of magnitude. Additional confidence in the results from the cylindrical model is given by the absolute spectral flux obtained from the simulations, as it agrees with the data from the experiment.

\subsection{Future work}

Although the results shown in Chapters 4 and 5 are encouraging, they are far from final and more work needs to be done on this subject in order to explore its possible 
applications.

Although the Cretin model has been benchmarked at OMEGA using the spectra of a vanadium plasma, extending the OpticalDepth platform to different elements would be of interest, in order to ensure a correct behaviour of the model. As detailed in Section 4.4, the set-up of these experiments allows one to obtain direct measurements of the opacity of the $y$ line. More importantly, it was shown that among the different codes used by the community, there are differences of up to a $\sim 50 \%$ for the oscillator strength of this line. Extending the OpticalDepth campaign to different elements would produce reliable ranges for this value, thus providing an additional check for the code-developing community while also helping experimentalists decide which code is more convenient for a particular experiment.

Ideally, the Cretin model presented in this work should be able to predict the changes in the $w / y$ ratio for a given temperature and density as a function of the plasma geometry. However, in the OpticalDepth experiments described in Chapter 4. both the temperature and geometry of the plasma changed independently at each timestep, with the ion density being determined by the geometry of the plasma. For this reason, the results shown in Figure 4.14 are temperature and geometry dependent. Nevertheless, it is possible to isolate the effects of the geometry with the same experimental set-up by using additional dots with a larger radius than that described in Chapter 4. For a radius of the dot up to $\sim 200 \mu \mathrm{m}$, a similar laser drive is obtained as the spot was designed to be flat over that area. Therefore, these larger dots would reach similar temperatures, and expand with velocities similar to those of the regular targets. In this case, at each timestep the larger dots would have the same temperature and density as in the regular case, only with a smaller aspect ratio (as the radius would be larger), thus allowing one to observe the changes in the spectra caused exclusively by a difference in the geometry. This approach was recently fielded at OMEGA, and the data is currently being analysed. 
Also, more work on the Dot Spectroscopy campaign is required. Figure 5.12 showed how after $t=11 \mathrm{~ns}$, the ion densities of the two dots considered in Chapter 5 were in agreement within error bars. This could indicate that some sort of equilibrium is reached with the surrounding plasma, thus meaning that the dots can be used not only to measure the electron temperature inside the hohlraum, but also as an indicator of the particle density. It was also mentioned that the disagreement in the densities for earlier times could be explained by the lack of data for the expansion of the thinnest target, as can be seen in Figure 5.7a, Acquiring more data for different thicknesses for the whole duration of the expansion is crucial to determine whether equilibrium is being reached or not.

In Section 5.6.3 it was also mentioned that the disagreement between the obtained densities and the predictions by HYDRA could be caused by the geometry of the dots' plasma not being perfectly cylindrical. In order to verify this, it would be necessary to perform new hydrodynamic simulations with the exact thicknesses of the dots in the experiment. It would then be possible to couple the Cretin model to these new simulations and study how the predictions for the $w / y$ ratio change as a result of the different geometries predicted by HYDRA, which would provide a deeper insight on the discrepancies between data and simulations. Furthermore, studying how different values of the electron flux limiter affect the outcome of the simulations, and comparing with the experimental results would limit the uncertainties in the parameters for the hydrodynamic simulations.

Lastly, in order to obtain a better cylindrical confinement in the Dot Spectroscopy experiments, so that more reliable measurements of the experimental ion density can be obtained, the possibility of burying the dot in a low-Z tamper in a similar manner as in the OpticalDepth campaign can be studied. If a tamper was implemented in such a way that the processes inside the hohlraum were not noticeably altered, maintaining a cylindrical shape of the dot during an $\mathrm{ICF}$ implosion experiment would provide 
more accurate measurements of the plasma conditions. These could be used to map the evolution of temperature and density in different regions of the hohlraum. This would not be a straightforward procedure, and extensive computational work would need to be done before its experimental implementation.

In conclusion, the work presented in this thesis shows how the angular distribution of the X-ray emission spectrum of plasmas depends on their geometry. In particular, theoretical, computational and experimental work with focus in the HED regime has been combined. In the 'OpticalDepth' experiments at the OMEGA laser, the evolution of uniform cylindrical plasmas was studied, which permitted benchmarking the results from theory and simulations. By combining these simulations with previous results from the Dot Spectroscopy campaign at NIF, it became possible to measure the ion density of the plasma through the ratio of optically thick to optically thin lines. This opens up the possibilities to further use these geometric effects as diagnostics in ICF experiments. This work provides the basis for the exploration of these geometric effects in HED experiments so that they can be used as a reliable diagnostic tool of the plasma conditions in the near future. 


\section{Acronyms}

CBET Cross Beam Energy Transfer. 10

CCD Charge-coupled Device. 76, 77, 105,106

CCR case-to-capsule ratio. 10

CH parylene. 5, 107, 108

DISC diagnostic insertion manipulator imaging streak camera. 105

DT Deuterium-Tritium. 2, 4, 12, 16

ENDF Evaluated Nuclear Data Files. 3

FAC Flexible Atomic Code [34]. 16

FWHM Full Width at Half Maximum. 86, 102

HDC High Density Carbon. 5, 108, 109

HED High Energy Density. 13, 14, 19, 21, 34, 51, 53, 55, 57, 58, 70, 71, 76, 98, 99, 103, 104, 124, 126, 128, 131

IAEA International Atomic Energy Agency. 3

ICF Inertial Confinement Fusion. 3, 6, 8, 9, 11, 14, 16, 19, 21, 52, 73, 99, 104, 107. 124, 128, 130, 131 
IFAR In Flight Aspect Ratio. 8, 9

IP Image Plate. 78, 105, 106

LEH Laser Entrance Hole. 107, 108, 110

LLE Laboratory for Laser Energetics. 16, 78

LLNL Lawrence Livermore National Laboratory. 18, 22, 107

LOS Line Of Sight. 29, 30, 32, 33, 39, 49, 72, 108, 125, 128

LPI Laser Plasma Interaction. 10, 11

MCP Microchannel Plate. 74,77, 105

mfp mean-free-path. 4, 29, 62

MSPEC Multi-Purpose Spectrometer. 76, 81, 83, 86, 88, 105, 127

NIC National Ignition Campaign. 18

NIF National Ignition Facility [23, 12, 18, 20, 22, 72, 99, 100, 105, 107, 109, 125, 128,131

NXS NIF X-ray Spectrometer. 100, 104, 106, 108, 111, 117, 123

ODE Ordinary Differential Equation. 28

PCD Photoconductive Detector. 72

PET pentaerythritol. 76, 77, 105

RT Rayleigh-Taylor. 8, 9

SBS Stimulated Brillouin Scattering. 10, 11 
SRS Stimulated Raman Scattering. 10, 11

TIM Ten-Inch Manipulator. 79

TO Target Orientation. $80,82,95$

XRD X-ray Diode. 72, 73,83

XRPINH X-Ray Pinhole Camera. 74, 75, 79, 80, 83, 84, 86, 88, 103, 105, 108, 110, 114, 116, 127 


\section{Bibliography}

[1] "International Atomic Energy Agency - Nuclear Data Section - Evaluated Nuclear Data Files." https://www-nds.iaea.org/exfor/endf.htm.

[2] J. Lawson, "Some criteria for a useful thermonuclear reactor," Atomic Energy Research Establishment, 1955.

[3] J. D. Lawson, "Some criteria for a power producing thermonuclear reactor," Proceedings of the physical society. Section B, vol. 70, no. 1, p. 6, 1957.

[4] J. D. Lindl, P. Amendt, R. L. Berger, S. G. Glendinning, S. H. Glenzer, S. W. Haan, R. L. Kauffman, O. L. Landen, and L. J. Suter, "The physics basis for ignition using indirect-drive targets on the National Ignition Facility," Physics of Plasmas, vol. 11, no. 2, pp. 339-154, 2004.

[5] J. Nuckolls, L. Wood, A. Thiessen, and G. Zimmerman, "Laser compression of matter to super-high densities: Thermonuclear (ctr) applications," Nature, vol. 239, no. 5368, pp. 139-142, 1972.

[6] R. Kidder and J. Zink, "Decoupling of corona and core of laser-heated pellets," Nuclear Fusion, vol. 12, no. 3, p. 325, 1972.

[7] R. Craxton, K. Anderson, T. Boehly, V. Goncharov, D. Harding, J. Knauer, R. McCrory, P. McKenty, D. Meyerhofer, J. Myatt, et al., "Direct-drive inertial 
confinement fusion: A review," Physics of Plasmas, vol. 22, no. 11, p. 110501, 2015.

[8] M. J. Edwards, P. K. Patel, J. D. Lindl, L. J. Atherton, S. H. Glenzer, S. W. Haan, J. D. Kilkenny, O. L. Landen, E. I. Moses, A. Nikroo, R. Petrasso, T. C. Sangster, P. T. Springer, S. Batha, R. Benedetti, L. Bernstein, R. Betti, D. L. Bleuel, T. R. Boehly, D. K. Bradley, J. A. Caggiano, D. A. Callahan, P. M. Celliers, C. J. Cerjan, K. C. Chen, D. S. Clark, G. W. Collins, E. L. Dewald, L. Divol, S. Dixit, T. Doeppner, D. H. Edgell, J. E. Fair, M. Farrell, R. J. Fortner, J. Frenje, M. G. Gatu Johnson, E. Giraldez, V. Y. Glebov, G. Grim, B. A. Hammel, A. V. Hamza, D. R. Harding, S. P. Hatchett, N. Hein, H. W. Herrmann, D. Hicks, D. E. Hinkel, M. Hoppe, W. W. Hsing, N. Izumi, B. Jacoby, O. S. Jones, D. Kalantar, R. Kauffman, J. L. Kline, J. P. Knauer, J. A. Koch, B. J. Kozioziemski, G. Kyrala, K. N. LaFortune, S. L. Pape, R. J. Leeper, R. Lerche, T. Ma, B. J. MacGowan, A. J. MacKinnon, A. Macphee, E. R. Mapoles, M. M. Marinak, M. Mauldin, P. W. McKenty, M. Meezan, P. A. Michel, J. Milovich, J. D. Moody, M. Moran, D. H. Munro, C. L. Olson, K. Opachich, A. E. Pak, T. Parham, H.-S. Park, J. E. Ralph, S. P. Regan, B. Remington, H. Rinderknecht, H. F. Robey, M. Rosen, S. Ross, J. D. Salmonson, J. Sater, D. H. Schneider, F. H. Séguin, S. M. Sepke, D. A. Shaughnessy, V. A. Smalyuk, B. K. Spears, C. Stoeckl, W. Stoeffl, L. Suter, C. A. Thomas, R. Tommasini, R. P. Town, S. V. Weber, P. J. Wegner, K. Widman, M. Wilke, D. C. Wilson, C. B. Yeamans, and A. Zylstra, "Progress towards ignition on the national ignition facility," Phys. Plasmas, vol. 20, no. 7, p. 070501, 2013.

[9] J. Meyer-ter Vehn, "On energy gain of fusion targets: the model of kidder and bodner improved," Nuclear Fusion, vol. 22, no. 4, p. 561, 1982. 
[10] J. Lindl, "Development of the indirect-drive approach to inertial confinement fusion and the target physics basis for ignition and gain," Phys. Plasmas, vol. 2, no. 11 , pp. 3933-4024, 1995.

[11] C. D. E. Fuentes, "Ignición rápida de blancos de fusión por confinamiento inercial mediante iones monoenergéticos," Proyecto Final de Carrera, Universidad Politécnica de Madrid, 2018.

[12] E. Storm, "Approach to high compression in inertial fusion," J. Fusion Energy, vol. 7 , no. 2, pp. 131-137, 1988.

[13] R. E. Olson, R. J. Leeper, A. Nobile, J. A. Oertel, G. A. Chandler, K. Cochrane, S. C. Dropinski, S. Evans, S. W. Haan, J. L. Kaae, J. P. Knauer, K. Lash, L. P. Mix, A. Nikroo, G. A. Rochau, G. Rivera, C. Russell, D. Schroen, R. J. Sebring, D. L. Tanner, R. E. Turner, and R. J. Wallace, "Shock propagation, preheat, and x-ray burnthrough in indirect-drive inertial confinement fusion ablator materials," Phys. Plasmas, vol. 11, no. 5, pp. 2778-2789, 2004.

[14] R. Betti and O. A. Hurricane, "Inertial-confinement fusion with lasers," Nature Physics, vol. 12, no. 5, pp. 435-448, 2016.

[15] A. B. Zylstra, S. A. Yi, S. MacLaren, J. Kline, G. Kyrala, J. Ralph, J. Bae, S. Batha, D. Callahan, K. Flippo, et al., "Beryllium capsule implosions at a case-to-capsule ratio of 3.7 on the national ignition facility," Physics of Plasmas, vol. 25, no. 10, p. 102704, 2018.

[16] D. Callahan, O. Hurricane, J. Ralph, C. Thomas, K. Baker, L. Benedetti, L. Berzak Hopkins, D. Casey, T. Chapman, C. Czajka, et al., "Exploring the limits of case-to-capsule ratio, pulse length, and picket energy for symmetric hohlraum drive on the national ignition facility laser," Physics of Plasmas, vol. 25 , no. 5, p. $056305,2018$. 
[17] S. W. Haan, S. M. Pollaine, J. D. Lindl, L. J. Suter, R. L. Berger, L. V. Powers, W. E. Alley, P. A. Amendt, J. A. Futterman, W. K. Levedahl, et al., "Design and modeling of ignition targets for the national ignition facility," Physics of Plasmas, vol. 2, no. 6, pp. 2480-2487, 1995.

[18] J. C. Fernández, S. Goldman, J. Kline, E. Dodd, C. Gautier, G. Grim, B. Hegelich, D. Montgomery, N. Lanier, H. Rose, et al., "Gas-filled hohlraum experiments at the national ignition facility," Physics of plasmas, vol. 13, no. 5, p. $056319,2006$.

[19] G. Hall, O. Jones, D. Strozzi, J. Moody, D. Turnbull, J. Ralph, P. Michel, M. Hohenberger, A. Moore, O. Landen, et al., "The relationship between gas fill density and hohlraum drive performance at the national ignition facility," Physics of Plasmas, vol. 24, no. 5, p. 052706, 2017.

[20] J. Moody, P. Datte, K. Krauter, E. Bond, P. Michel, S. Glenzer, L. Divol, C. Niemann, L. Suter, N. Meezan, et al., "Backscatter measurements for nif ignition targets," Review of Scientific Instruments, vol. 81, no. 10, p. 10D921, 2010.

[21] S. Glenzer, B. MacGowan, N. Meezan, P. Adams, J. Alfonso, E. Alger, Z. Alherz, L. Alvarez, S. Alvarez, P. Amick, et al., "Demonstration of ignition radiation temperatures in indirect-drive inertial confinement fusion hohlraums," Physical review letters, vol. 106, no. 8, p. 085004, 2011.

[22] P. Michel, L. Divol, E. Dewald, J. Milovich, M. Hohenberger, O. Jones, L. B. Hopkins, R. Berger, W. Kruer, and J. Moody, "Multibeam stimulated raman scattering in inertial confinement fusion conditions," Physical review letters, vol. 115, no. 5, p. 055003, 2015. 
[23] G. H. Miller, E. I. Moses, and C. R. Wuest, "The National Ignition Facility," Opt. Eng., vol. 43, pp. $43-43-13,2004$.

[24] J. P. Apruzese, K. G. Whitney, J. Davis, and P. C. Kepple, "K-shell line ratios and powers for diagnosing cylindrical plasmas of neon, aluminum, argon, and titanium," J. Quant. Spectrosc. Radiat. Transfer, vol. 57, no. 1, pp. $41-61$, 1997.

[25] J. P. Apruzese, D. Duston, and J. Davis, "K-shell aluminum resonance line ratios for plasma diagnosis using spot spectroscopy," J. Quant. Spectrosc. Radiat. Transfer, vol. 36, no. 4, pp. 339 - 344, 1986.

[26] C. A. Back, D. H. Kalantar, R. L. Kauffman, R. W. Lee, B. K. MacGowan, D. S. Montgomery, L. V. Powers, T. D. Shepard, G. F. Stone, and L. J. Suter, "Measurements of electron temperature by spectroscopy in hohlraum targets," Phys. Rev. Lett., vol. 77, no. 21, 1996.

[27] T. D. Shepard, C. A. Back, D. H. Kalantar, R. L. Kauffman, C. J. Keane, D. E. Klem, B. F. Lasinski, B. J. MacGowan, L. V. Powers, L. J. Suter, R. E. Turner, B. H. Failor, and W. W. Hsing, "Isoelectronic x-ray spectroscopy to determine electron temperatures in lon-scale-length inertial-confinement-fusion plasmas," Phys. Rev. E, vol. 53, no. 5, 1996.

[28] M. A. Barrios, D. A. Liedahl, M. B. Schneider, O. Jones, G. V. Brown, S. P. Regan, K. B. Fournier, A. S. Moore, J. S. Ross, O. Landen, R. L. Kauffman, A. Nikroo, J. Kroll, J. Jaquez, H. Huang, S. B. Hansen, D. A. Callahan, D. E. Hinkel, D. Bradley, and J. D. Moody, "Electron temperature measurements inside the ablating plasma of gas-filled hohlraums at the National Ignition Facility," Phys. Plasmas, vol. 23, no. 5, p. 056307, 2016. 
[29] M. A. Barrios, J. D. Moody, L. J. Suter, M. Sherlock, H. Chen, W. Farmer, J. Jaquez, O. Jones, R. L. Kauffman, J. D. Kilkenny, J. Kroll, O. L. Landen, D. A. Liedahl, S. A. Maclaren, N. B. Meezan, A. Nikroo, M. B. Schneider, D. B. Thron, K. Widmann, and G. Pérez-Callejo, "Developing an experimental basis for understanding transport in NIF hohlraum plasmas," Phys. Rev. Lett., 2018.

[30] N. R. Council, Frontiers in High Energy Density Physics: The X-Games of Contemporary Science. Washington, DC: The National Academies Press, 2003.

[31] H. A. Scott, "CRETIN - a radiative transfer capability for laboratory plasmas," J. Quant. Spectrosc. Radiat. Transfer, vol. 71, no. 2, pp. 689 - 701, 2001. Radiative Properties of Hot Dense Matter.

[32] H. Scott and S. Hansen, "Advances in nlte modeling for integrated simulations," High Energy Density Physics, vol. 6, no. 1, pp. 39-47, 2010.

[33] H. Scott and R. Mayle, "Glf-a simulation code for x-ray lasers," Applied Physics B, vol. 58, no. 1, pp. 35-43, 1994.

[34] M. F. Gu, "The flexible atomic code," Canadian Journal of Physics, vol. 86, no. 5, pp. 675-689, 2008.

[35] T. R. Boehly, D. L. Brown, R. S. Craxton, R. L. Keck, J. P. Knauer, J. H. Kelly, T. J. Kessler, S. A. Kumpan, S. J. Loucks, S. A. Letzring, F. J. Marshall, R. L. McCrory, S. F. B. Morse, W. Seka, J. M. Soures, and C. P. Verdon, "Initial performance results of the OMEGA laser system," Opt. Commun., vol. 133, no. 1 , pp. $495-506,1997$.

[36] T. Boehly, R. Craxton, T. Hinterman, J. Kelly, T. Kessler, S. Kumpan, S. Letzring, R. McCrory, S. Morse, W. Seka, et al., "The upgrade to the omega laser system," Review of Scientific Instruments, vol. 66, no. 1, pp. 508-510, 1995. 
[37] L. Waxer, D. Maywar, J. Kelly, T. Kessler, B. Kruschwitz, S. Loucks, R. McCrory, D. Meyerhofer, S. Morse, C. Stoeckl, et al., "High-energy petawatt capability for the omega laser," Optics and photonics news, vol. 16, no. 7, pp. 30-36, 2005 .

[38] J. Kelly, L. Waxer, V. Bagnoud, I. Begishev, J. Bromage, B. Kruschwitz, T. Kessler, S. Loucks, D. Maywar, R. McCrory, et al., "Omega ep: High-energy petawatt capability for the omega laser facility," in Journal de Physique IV (Proceedings), vol. 133, pp. 75-80, EDP sciences, 2006.

[39] OMEGA Upgrade Operations Manual, vol. 1 - System description, ch. 1 - System Overview. 1le.rochester.edu, 2003.

[40] J. Soures, R. McCrory, C. Verdon, A. Babushkin, R. Bahr, T. Boehly, R. Boni, D. Bradley, D. Brown, R. Craxton, et al., "Direct-drive laser-fusion experiments with the omega, 60-beam, 40 kj, ultraviolet laser system," Physics of Plasmas, vol. 3, no. 5, pp. 2108-2112, 1996.

[41] M. Hohenberger, P.-Y. Chang, G. Fiksel, J. P. Knauer, R. Betti, F. J. Marshall, D. D. Meyerhofer, F. H. Séguin, and R. D. Petrasso, "Inertial confinement fusion implosions with imposed magnetic field compression using the omega laser," Physics of Plasmas, vol. 19, no. 5, p. 056306, 2012.

[42] T. J. Murphy, J. M. Wallace, N. D. Delamater, C. W. Barnes, P. Gobby, A. A. Hauer, E. L. Lindman, G. Magelssen, J. B. Moore, J. A. Oertel, R. Watt, O. L. Landen, P. Amendt, M. Cable, C. Decker, B. A. Hammel, J. A. Koch, L. J. Suter, R. E. Turner, R. J. Wallace, F. J. Marshall, D. Bradley, R. S. Craxton, R. Keck, J. P. Knauer, R. Kremens, and J. D. Schnittman, "Indirect drive experiments utilizing multiple beam cones in cylindrical hohlraums on omega," Physics of Plasmas, vol. 5, no. 5, pp. 1960-1965, 1998. 
[43] J. P. Knauer, R. Betti, D. K. Bradley, T. R. Boehly, T. J. B. Collins, V. N. Goncharov, P. W. McKenty, D. D. Meyerhofer, V. A. Smalyuk, C. P. Verdon, S. G. Glendinning, D. H. Kalantar, and R. G. Watt, "Single-mode, rayleigh-taylor growth-rate measurements on the omega laser system," Physics of Plasmas, vol. 7 , no. 1 , pp. 338-345, 2000.

[44] E. C. Harding, J. F. Hansen, O. A. Hurricane, R. P. Drake, H. F. Robey, C. C. Kuranz, B. A. Remington, M. J. Bono, M. J. Grosskopf, and R. S. Gillespie, "Observation of a kelvin-helmholtz instability in a high-energy-density plasma on the omega laser," Phys. Rev. Lett., vol. 103, p. 045005, Jul 2009.

[45] P. Tzeferacos, A. Rigby, A. Bott, A. Bell, R. Bingham, A. Casner, F. Cattaneo, E. Churazov, J. Emig, F. Fiuza, et al., "Laboratory evidence of dynamo amplification of magnetic fields in a turbulent plasma," Nature communications, vol. 9, no. 1, pp. 1-8, 2018.

[46] J. Lindl, O. Landen, J. Edwards, E. Moses, and N. team, "Review of the national ignition campaign 2009-2012," Physics of Plasmas, vol. 21, no. 2, p. 020501, 2014.

[47] "Nif sets new laser energy record." https://www.llnl.gov/news/nif-sets-newlaser-energy-record.

[48] M. L. Spaeth, K. Manes, D. Kalantar, P. Miller, J. Heebner, E. Bliss, D. Spec, T. Parham, P. Whitman, P. Wegner, et al., "Description of the nif laser," Fusion Science and Technology, vol. 69, no. 1, pp. 25-145, 2016.

[49] J. Moody, H. Robey, P. Celliers, D. Munro, D. Barker, K. Baker, T. Döppner, N. Hash, L. Berzak Hopkins, K. LaFortune, et al., "Early time implosion symmetry from two-axis shock-timing measurements on indirect drive nif experiments," Physics of Plasmas, vol. 21, no. 9, p. 092702, 2014. 
[50] J. Rygg, O. Jones, J. Field, M. Barrios, L. Benedetti, G. Collins, D. Eder, M. Edwards, J. Kline, J. Kroll, et al., "2d x-ray radiography of imploding capsules at the national ignition facility," Physical review letters, vol. 112, no. 19, p. 195001, 2014.

[51] D. Hinkel, L. B. Hopkins, T. Ma, J. Ralph, F. Albert, L. Benedetti, P. Celliers, T. Döppner, C. Goyon, N. Izumi, et al., "Development of improved radiation drive environment for high foot implosions at the national ignition facility," Physical review letters, vol. 117, no. 22, p. 225002, 2016.

[52] P. Amendt, J. S. Ross, J. L. Milovich, M. Schneider, E. Storm, D. A. Callahan, D. Hinkel, B. Lasinski, D. Meeker, P. Michel, et al., "Low-adiabat rugby hohlraum experiments on the national ignition facility: Comparison with highflux modeling and the potential for gas-wall interpenetration," Physics of Plasmas, vol. 21, no. 11, p. 112703, 2014.

[53] J. Ross, H. Robey, H. Chen, N. Izumi, S. Johnson, T. Ma, N. Meezan, M. Millot, J. Moody, A. Moore, et al., "Initial experimental results of indirectly-driven icf implosions in the i-raum," in APS Meeting Abstracts, 2018.

[54] G. Pérez-Callejo, J. Wark, and S. Rose, "Radiation transfer in cylindrical, toroidal and hemi-ellipsoidal plasmas," Journal of Quantitative Spectroscopy and Radiative Transfer, vol. 235C, pp. 24-30, 2019.

[55] G. Pérez-Callejo, D. A. Liedahl, M. B. Schneider, S. J. Rose, and J. S. Wark, "The use of geometric effects in diagnosing ion density in ICF-related dot spectroscopy experiments," High Energy Density Physics, vol. 30, pp. 45 - 51, 2019.

[56] G. Pérez-Callejo, L. C. Jarrott, D. A. Liedahl, E. V. Marley, G. E. Kemp, R. F. Heeter, J. A. Emig, M. E. Foord, K. Widmann, J. Jaquez, H. Huang, S. J. Rose, J. S. Wark, and M. B. Schneider, "Laboratory measurements of geometrical 
effects in the x-ray emission of optically thick lines for icf diagnostics," Physics of Plasmas, vol. 26, no. 6, p. 063302, 2019.

[57] M. M. Marinak, R. E. Tipton, O. L. Landen, T. J. Murphy, P. Amendt, S. W. Haan, S. P. Hatchett, C. J. Keane, R. McEachern, and R. Wallace, "Threedimensional simulations of nova high growth factor capsule implosion experiments," Physics of Plasmas, vol. 3, no. 5, pp. 2070-2076, 1996.

[58] D. Mihalas, Stellar Atmospheres - Second Edition. San Francisco: W. H. Freeman \& Company, 1978.

[59] T. Holstein, "Imprisonment of resonance radiation in gases," Phys. Rev., vol. 72, pp. 1212-1233, Dec 1947.

[60] E. R. Capriotti, "Mean Escape Probabilities of Line Photons.," Astrophys. J., vol. 142, p. 1101, Oct 1965.

[61] J. P. Apruzese, "Direct solution of the equation of transfer using frequencyand angle-averaged photon-escape probabilities for spherical and cylindrical geometries," J. Quant. Spectrosc. Radiat. Transfer, vol. 25, no. 5, pp. $419-425$, 1981.

[62] J. J. MacFarlane, "Collisional-radiative equilibirum (cre) model for the conrad radiation-hydrodynamics code," University of Wisconsin Fusion Technology Institute Report, vol. UWFDM-937, Dec. 1993.

[63] S. J. Rose, "The non-LTE excitation/ionization code GALAXY," Journal of Physics B: Atomic, Molecular and Optical Physics, vol. 31, pp. 2129-2144, may 1998.

[64] S. Pestehe and G. Tallents, "Escape factors for laser-plasmas," Journal of Quantitative Spectroscopy and Radiative Transfer, vol. 72, no. 6, pp. 853-878, 2002. 
[65] G. J. Philips, J. S. Wark, F. M. Kerr, S. J. Rose, and R. W. Lee, "Escape factors in zero-dimensional radiation-transfer codes," High Energy Density Phys., vol. 4, pp. 18-25, Nov. 2008.

[66] P. A. M. Dirac, The Collected Works of P. A. M. Dirac 1924-1948, ch. Approximate rate of neutron multiplication for a solid of arbitrary shape and uniform density, I: General theory, pp. 1115-1128. Oxford University Press, 1995.

[67] P. A. M. Dirac, K. Fuchs, R. Peierls, , and P. Preston, The Collected Works of P. A. M. Dirac 1924-1948, ch. Approximate rate of neutron multiplication for a solid of arbitrary shape and uniform density, II: Application to the oblate spheroid, hemisphere and oblate hemispheroid, pp. 1129-1145. Oxford University Press, 1995.

[68] J. Lambert, Photometria sive de Mensura et gradibus luminis, colorum et umbrae. sumptibus viduae E. Klett, 1760.

[69] Beer, "Bestimmung der absorption des rothen lichts in farbigen flüssigkeiten," Annalen der Physik, vol. 162, no. 5, pp. 78-88, 1852.

[70] A. K. Bhatia and S. O. Kastner, "The optically thick fe xvii spectrum: X-ray, extreme-ultraviolet, and forbidden line ratios," Astrophys. J., vol. 516, no. 1, p. $482,1999$.

[71] A. K. Bhatia and J. L. R. Saba, "Resonance scattering of fe xvii x-ray and extreme-ultraviolet lines," Astrophys. J., vol. 563, no. 1, p. 434, 2001.

[72] S. O. Kastner and A. K. Bhatia, "Optically thin and thick fe xv spectrum: Effect of self-absorption on the 284.16 Å resonance line," Astrophys. J., vol. 553, no. 1, p. 421, 2001. 
[73] F. M. Kerr, S. J. Rose, J. S. Wark, and F. P. Keenan, "Enhancement of optically thick to thin line intensities in solar and stellar coronal plasmas through radiative transfer effects: an angularly resolved study," Astrophys. J., vol. 613, pp. L181-L184, Oct. 2004.

[74] F. M. Kerr, S. J. Rose, and J. S. Wark, "An analytic geometry-variant approach to line ratio enhancement above the optically thin limit," Astrophys. J., vol. 629, p. 1091, Jan. 2005.

[75] F. M. Kerr, A. Gouveia, O. Renner, S. J. Rose, H. A. Scott, and J. S. Wark, "Line radiation effects in laboratory and astrophysical plasmas," J. Quant. Spectrosc. Radiat. Transfer, vol. 99, no. 1, pp. 363-369, 2006.

[76] P. Hatfield, "Using line intensity ratios to determine the geometry of plasma in stars via their apparent areas," High Energy Density Phys., vol. 6, no. 3, pp. $301-304,2010$.

[77] D. M. Chambers, P. A. Pinto, J. Hawreliak, I. R. Al'Miev, A. Gouveia, P. Sondhauss, E. Wolfrum, J. S. Wark, S. H. Glenzer, R. W. Lee, P. E. Young, O. Renner, R. S. Marjoribanks, and S. Topping, "K-shell spectroscopy of an independently diagnosed uniaxially expanding laser-produced aluminum plasma," Phys. Rev. E, vol. 66, p. 026410, Aug 2002.

[78] R. C. Mancini, J. E. Bailey, J. F. Hawley, T. Kallman, M. Witthoeft, S. J. Rose, and H. Takabe, "Accretion disk dynamics, photoionized plasmas, and stellar opacities," Phys. Plasmas, vol. 16, no. 4, p. 041001, 2009.

[79] M. Abramowitz and I. A. Stegun, Handbook of Mathematical Functions: With Formulas, Graphs, and Mathematical Tables. Applied mathematics series, Dover Publications, 1965. 
[80] G. J. Tallents, "Opacity effects on laser-produced plasma radiation sources," Journal of Applied Physics, vol. 126, no. 8, p. 083302, 2019.

[81] L. Heroux, "A spectroscopic determination of electron temperature in a high temperature plasma," Proceedings of the Physical Society, vol. 83, no. 1, p. 121, 1964.

[82] K. P. Dere, H. E. Mason, K. G. Widing, and A. K. Bhatia, "XUV electron density diagnostics for solar flares," Astrophys. J. Suppl. Ser., vol. 40, pp. 341364, June 1979.

[83] J. D. Kilkenny, R. W. Lee, M. H. Key, and J. G. Lunney, "X-ray spectroscopic diagnosis of laser-produced plasmas, with emphasis on line broadening," Phys. Rev. A ., vol. 22, pp. 2746-2760, Dec. 1980.

[84] R. S. Marjoribanks, M. C. Richardson, P. A. Jaanimagi, and R. Epstein, "Electron-temperature measurement in laser-produced plasmas by the ratio of isoelectronic line intensities," Phys. Rev., vol. 46, Aug. 1992.

[85] C. J. Keane, B. A. Hammel, D. R. Kania, J. D. Kilkenny, R. W. Lee, A. L. Osterheld, L. J. Suter, R. C. Mancini, C. F. Hooper, and N. D. Delamater, "Xray spectroscopy of high-energy density inertial confinement fusion plasmas," Phys. Fluids B: Plasma Phys., vol. 5, no. 9, pp. 3328-3336, 1993.

[86] J. Ashkenazy, R. Kipper, and M. Caner, "Spectroscopic measurements of electron density of capillary plasma based on stark broadening of hydrogen lines," Phys. Rev. A, vol. 43, pp. 5568-5574, May 1991.

[87] C. A. Morgan, H. R. Griem, and R. C. Elton, "Spectroscopic measurements of electron density and temperature in polyacetal-capillary-discharge plasmas," Phys. Rev. E, vol. 49, pp. 2282-2290, Mar 1994. 
[88] M. Ivković, S. Jovićević, and N. Konjević, "Low electron density diagnostics: development of optical emission spectroscopic techniques and some applications to microwave induced plasmas," Spectrochim. Acta, Part B, vol. 59, no. 5, pp. $591-605,2004$.

[89] G. J. Bastiaans and R. A. Mangold, "The calculation of electron density and temperature in ar spectroscopic plasmas from continuum and line spectra," Spectrochim. Acta, Part B, vol. 40, no. 7, pp. 885 - 892, 1985.

[90] E. A. Den Hartog, T. R. O'Brian, and J. E. Lawler, "Electron temperature and density diagnostics in a helium glow discharge," Phys. Rev. Lett., vol. 62, pp. 1500-1503, Mar 1989.

[91] A. H. Gabriel, "Dielectronic satellite spectra for highly-charged helium-like ion lines," Mon. Not. R. Astron. Soc., vol. 160, pp. 99-119, 1972.

[92] A. H. Gabriel and C. Jordan, "Interpretation of solar helium-like ion line intensities," Mon. Not. R. Astron. Soc., vol. 145, no. 2, pp. 241-248, 1969.

[93] G. A. Doschek and J. F. Meekins, "The helium-like calcium, silicon, and sulfur lines during the decay of a large flare," Sol. Phys., vol. 13, pp. 220-225, Jul 1970.

[94] G. R. Blumenthal, G. W. F. Drake, and W. H. Tucker, "Ratio of line intensities in helium-like ions as a density indicator.," Astrophys. J., vol. 172, p. 205, Feb. 1972.

[95] L. W. Acton, R. C. Catura, A. J. Meyerott, C. J. Wolfson, and J. L. Culhane, "Coronal survey in x-rays of o vii and ne ix," Sol. Phys., vol. 26, pp. 183-201, Sep 1972. 
[96] F. P. Keenan, S. S. Tayal, and A. E. Kingston, "Theoretical neix line ratios compared to solar observations," Sol. Phys., vol. 94, pp. 85-89, Aug 1984.

[97] F. P. Keenan, W. A. Feibelman, and K. A. Berrington, "Improved calculations for the C III 1907,1909 and Si III 1883,1892 electron density sensitive emissionline ratios, and a comparison with IUE observations," Astrophys. J., vol. 389, pp. 443-446, Apr. 1992.

[98] Porquet, D., Mewe, R., Dubau, J., Raassen, A. J. J., and Kaastra, J. S., "Line ratios for helium-like ions: Applications to collision-dominated plasmas*," Astron. Astrophys., vol. 376, no. 3, pp. 1113-1122, 2001.

[99] Ness, J.-U., Mewe, R., Schmitt, J. H. M. M., Raassen, A. J. J., Porquet, D., Kaastra, J. S., van der Meer, R. L. J., Burwitz, V., and Predehl, P., "Heliumlike triplet density diagnostics - applications to chandra-letgs x-ray observations of capella and procyon," Astron. Astrophys., vol. 367, no. 1, pp. 282-296, 2001.

[100] Ness, J.-U., Schmitt, J. H. M. M., Burwitz, V., Mewe, R., Raassen, A. J. J., van der Meer, R. L. J., Predehl, P., and Brinkman, A. C., "Coronal density diagnostics with helium-like triplets: Chandra-letgs observations of algol, capella, procyon, i, n a\&b, ux ari, ad leo, yy gem, and hr9," Astron. Astrophys., vol. 394, no. 3, pp. 911-926, 2002.

[101] D. Porquet, J. Dubau, and N. Grosso, "He-like ions as practical astrophysical plasma diagnostics: From stellar coronae to active galactic nuclei," Space Sci. Rev., vol. 157, pp. 103-134, Dec 2010.

[102] A. V. Vinogradov, I. Y. Skobelev, and E. A. Yukov, "Determination of plasma density from spectra of heliumlike ions," Sov. J. Quantum Electron., vol. 5, no. 6, p. 630, 1975 . 
[103] V. P. Shevelko, I. Y. Skobelev, and A. V. Vinogradov, "Density-dependent lines of one- and two-electron ions in diagnostics of laboratory plasma. i. the rates of collision relaxation of excited levels," Phys. Scr., vol. 16, no. 3-4, p. 123, 1977.

[104] I. Y. Skobelev, A. V. Vinogradov, and E. A. Yukov, "Density-dependent lines of one- and two-electron ions in diagnostics of laboratory plasma. II. intensity line ratios of hydrogenlike, heliumlike and oxygenlike multicharged ions," Phys. Scr., vol. 18, no. 2, p. 78, 1978.

[105] V. A. Boiko, A. Y. Chugunov, A. Y. Faenov, S. A. Pikuz, I. Y. Skobelev, A. V. Vinogradov, and E. A. Yukov, "Anomalous intensity ratios of the resonance to intercombination lines of He-like ions in Nd- and CO 2 -laser-produced plasmas," J. Phys. B: At. Mol. Phys., vol. 12, no. 2, p. 213, 1979.

[106] V. A. Boiko, A. Y. Faenov, and S. A. Pikuz, "X-ray spectroscopy of multiplycharged ions from laser plasmas," J. Quant. Spectrosc. Radiat. Transfer, vol. 19, no. 1 , pp. $11-50,1978$.

[107] D. Duston and J. Davis, "Line emission from hot, dense, aluminum plasmas," Phys. Rev. A, vol. 21, pp. 1664-1676, May 1980.

[108] M. Chaker, J. C. Kieffer, J. P. Matte, H. Pépin, P. Audebert, P. Maine, D. Strickland, P. Bado, and G. Mourou, "Interaction of a 1 psec laser pulse with solid matter," Phys. Fluids B: Plasma Phys., vol. 3, no. 1, pp. 167-175, 1991.

[109] H. Fiedorowicz, A. Bartnik, Z. Patron, and P. Parys, "X-ray emission from laser-irradiated gas puff targets," Appl. Phys. Lett., vol. 62, no. 22, pp. 27782780, 1993. 
[110] J. G. Doyle and J. L. Schwob, "Intercombination to resonance line intensity ratio for he-like oxygen and carbon ions in tfr tokamak plasmas," J. Phys. B: At. Mol. Phys., vol. 15, no. 5, p. 813, 1982.

[111] M. Bitter, K. W. Hill, S. v. Goeler, W. Stodiek, P. Beiersdorfer, J. E. Rice, and A. Ince-Cushman, "Measurements of the intercombination and forbidden lines from the spectra of helium-like ions in tokamaks and electron beam ion traps," Can. J. Phys., vol. 86, no. 1, pp. 291-306, 2008.

[112] S. B. Hansen, J. Bauche, C. Bauche-Arnoult, and M. F. Gu, "Hybrid atomic models for spectroscopic plasma diagnostics," High Energy Density Phys., vol. 3, no. 1, pp. 109 - 114, 2007. Radiative Properties of Hot Dense Matter.

[113] H. R. Griem, Plasma Spectroscopy. McGraw-Hill, 1964.

[114] H. R. Griem, Spectral Line Broadening by Plasmas. Academic Press, 1974.

[115] D. Salzmann, Atomic Physics in Hot Plasmas. Oxford University Press, 1998.

[116] W. J. Gray, M. E. Foord, M. B. Schneider, M. A. Barrios, G. V. Brown, R. F. Heeter, L. C. Jarrott, D. A. Liedahl, E. V. Marley, C. W. Mauche, and K. Widmann, "Investigation of the hydrodynamics and emission of a laser heated tamped high-z target," Phys. Plasmas, vol. 25, 2018.

[117] J. H. Holland, "Adaptation in natural and artificial systems," Ann Arbor: University of Michigan Press, 1975.

[118] M. F. Kasim, T. P. Galligan, J. Topp-Mugglestone, G. Gregori, and S. M. Vinko, "Inverse problem instabilities in large-scale modeling of matter in extreme conditions," Physics of Plasmas, vol. 26, no. 11, p. 112706, 2019. 
[119] H. G. Ahlstrom, L. W. Coleman, F. Rienecker, and V. W. Slivinsky, "Diagnostics of shiva nova high-yield thermonuclear events," J. Opt. Soc. Am., vol. 68, pp. 1731-1741, Dec 1978.

[120] H. N. Kornblum, R. L. Kauffman, and J. A. Smith, "Measurement of 0.1-3-kev x rays from laser plasmas," Review of Scientific Instruments, vol. 57, no. 8, pp. 2179-2181, 1986.

[121] K. M. Campbell, F. A. Weber, E. L. Dewald, S. H. Glenzer, O. L. Landen, R. E. Turner, and P. A. Waide, "OMEGA dante soft x-ray power diagnostic component calibration at the national synchrotron light source," Review of Scientific Instruments, vol. 75, no. 10, pp. 3768-3771, 2004.

[122] C. Sorce, J. Schein, F. Weber, K. Widmann, K. Campbell, E. Dewald, R. Turner, O. Landen, K. Jacoby, P. Torres, and D. Pellinen, "Soft x-ray power diagnostic improvements at the OMEGA laser facility," Review of Scientific Instruments, vol. 77, no. 10, p. 10E518, 2006.

[123] J. L. Kline, K. Widmann, A. Warrick, R. Olson, C. Thomas, A. Moore, L. Suter, O. Landen, D. Callahan, S. Azevedo, et al., "The first measurements of soft Xray flux from ignition scale hohlraums at the national ignition facility using dante," Review of Scientific Instruments, vol. 81, no. 10, p. 10E321, 2010.

[124] J. L. Kline, "Measurements of soft x-ray flux from large scale hohlraums at the national ignition facility," tech. rep., Los Alamos National Lab.(LANL), Los Alamos, NM (United States), 2011.

[125] R. Marrs, K. Widmann, G. Brown, R. Heeter, S. MacLaren, M. May, A. Moore, and M. Schneider, "Use of a priori spectral information in the measurement of x-ray flux with filtered diode arrays," Review of Scientific Instruments, vol. 86, no. 10, p. 103511, 2015. 
[126] J. L. Wiza et al., "Microchannel plate detectors," Nucl. Instrum. Methods, vol. 162, no. 1-3, pp. 587-601, 1979.

[127] G. Fraser, M. Barstow, M. Whiteley, and A. Wells, "Enhanced soft x-ray detection efficiencies for imaging microchannel plate detectors," Nature, vol. 300, no. 5892, p. 509, 1982.

[128] G. Fraser, M. Barstow, J. Pearson, M. Whiteley, and M. Lewis, "The soft x-ray detection efficiency of coated microchannel plates," Nuclear Instruments and Methods in Physics Research, vol. 224, no. 1-2, pp. 272-286, 1984.

[129] J. Kilkenny, "High speed proximity focused x-ray cameras," Laser and Particle Beams, vol. 9, no. 1, pp. 49-69, 1991.

[130] D. Bradley, P. Bell, O. Landen, J. Kilkenny, and J. Oertel, "Development and characterization of a pair of 30-40 ps x-ray framing cameras," Review of scientific instruments, vol. 66, no. 1, pp. 716-718, 1995.

[131] F. Ze, R. Kauffman, J. Kilkenny, J. Wielwald, P. Bell, R. Hanks, J. Stewart, D. Dean, J. Bower, and R. Wallace, "A new multichannel soft x-ray framing camera for fusion experiments," Review of scientific instruments, vol. 63, no. 10, pp. 5124-5126, 1992.

[132] D. Bradley, P. Bell, J. Kilkenny, R. Hanks, O. Landen, P. Jaanimagi, P. McKenty, and C. Verdon, "High-speed gated x-ray imaging for icf target experiments," Review of scientific instruments, vol. 63, no. 10, pp. 4813-4817, 1992.

[133] B. Henke, H. Yamada, and T. Tanaka, "Pulsed plasma source spectrometry in the 80-8000-ev x-ray region," Review of scientific instruments, vol. 54, no. 10, pp. 1311-1330, 1983. 
[134] B. Henke and P. Jaanimagi, "Two-channel, elliptical analyzer spectrograph for absolute, time-resolving time-integrating spectrometry of pulsed x-ray sources in the 100-10 000-ev region," Review of scientific instruments, vol. 56, no. 8, pp. 1537-1552, 1985.

[135] R. F. Heeter, J. A. Emig, K. B. Fournier, S. B. Hansen, M. J. May, and B. K. F. Young, "X-ray spectroscopy with elliptical crystals and face-on framing cameras," Rev. Sci. Instrum., vol. 75, pp. 3762-3764, Oct. 2004.

[136] R. E. Marrs, G. V. Brown, J. A. Emig, and R. F. Heeter, "System for calibrating the energy-dependent response of an elliptical bragg-crystal spectrometer)," Rev. Sci. Instrum., vol. 85, pp. 11DS26 1-5, Sept. 2014.

[137] M. Rosenberg, D. Thorn, N. Izumi, D. Williams, M. Rowland, G. Torres, M. Haugh, P. Hillyard, N. Adelman, T. Schuler, et al., "Image-plate sensitivity to x rays at 2 to $60 \mathrm{kev,"} \mathrm{Review} \mathrm{of} \mathrm{Scientific} \mathrm{Instruments,} \mathrm{vol.} \mathrm{90,} \mathrm{no.} \mathrm{1,}$ p. 013506, 2019.

[138] M. Rosenberg, D. Thorn, N. Izumi, D. Williams, M. Rowland, G. Torres, M. Haugh, P. Hillyard, N. Adelman, T. Schuler, et al., "Erratum: "image-plate sensitivity to x rays at 2 to 60 kev" [rev. sci. instrum. 90, 013506 (2019)]," Review of Scientific Instruments, vol. 90, no. 2, p. 029902, 2019.

[139] H. Jeffreys, "Some tests of significance, treated by the theory of probability," Mathematical Proceedings of the Cambridge Philosophical Society, vol. 31, no. 2, p. $203-222,1935$.

[140] H. Jeffreys, The theory of probability. Oxford, 1961.

[141] R. E. Kass and A. E. Raftery, "Bayes factors," J. Am. Stat. Assoc., vol. 90, pp. 773-795, June 1995. 
[142] M. Edwards, D. Whittaker, P. Mistry, N. Booth, G. Pert, G. Tallents, B. Rus, T. Mocek, M. Koslová, C. McKenna, et al., "Opacity measurements of a hot iron plasma using an x-ray laser," Physical review letters, vol. 97, no. 3, p. 035001, 2006.

[143] T. R. Preston, S. M. Vinko, O. Ciricosta, P. Hollebon, H.-K. Chung, G. L. Dakovski, J. Krzywinski, M. Minitti, T. Burian, J. Chalupský, V. Hájková, L. Juha, V. Vozda, U. Zastrau, R. W. Lee, and J. S. Wark, "Measurements of the $k$-shell opacity of a solid-density magnesium plasma heated by an x-ray free-electron laser," Phys. Rev. Lett., vol. 119, p. 085001, Aug 2017.

[144] H. K. Chung, M. H. Chen, W. L. Morgan, Y. Ralchenko, and R. W. Lee, "Flychk: Generalized population kinetics and spectral model for rapid spectroscopic analysis for all elements," High Energy Density Phys., vol. 1, pp. 3-12, Dec. 2005.

[145] C. E. Max, Physics of Laser Fusion. Volume I. Theory of the Coronal Plasma in Laser Fusion Targets. Lawrence Livermore National Laboratory, December 1982.

[146] J. Christiansen, D. Ashby, and K. Roberts, "Medusa a one-dimensional laser fusion code," Computer Physics Communications, vol. 7, no. 5, pp. $271-287$, 1974.

[147] A. Djaoui and S. J. Rose, "Calculation of the time-dependent excitation and ionization in a laser-produced plasma," Journal of Physics B: Atomic, Molecular and Optical Physics, vol. 25, pp. 2745-2762, jun 1992.

[148] G. Mourou and W. Knox, "A picosecond jitter streak camera," Applied Physics Letters, vol. 36, no. 8, pp. 623-626, 1980. 
[149] M. Murnane, H. Kapteyn, and R. Falcone, "X-ray streak camera with 2 ps response," Applied physics letters, vol. 56, no. 20, pp. 1948-1950, 1990.

[150] D. Bradley, B. Liddy, and W. Sleat, "Direct linear measurement of ultrashort light pulses with a picosecond streak camera," Optics Communications, vol. 2, no. 8, pp. 391-395, 1971.

[151] Z. Chang, A. Rundquist, J. Zhou, M. Murnane, H. Kapteyn, X. Liu, B. Shan, J. Liu, L. Niu, M. Gong, et al., "Demonstration of a sub-picosecond x-ray streak camera," Applied physics letters, vol. 69, no. 1, pp. 133-135, 1996.

[152] O. Landen, T. Boehly, D. Bradley, D. Braun, D. Callahan, P. Celliers, G. Collins, E. Dewald, L. Divol, S. Glenzer, et al., "Capsule performance optimization in the national ignition campaign," Physics of Plasmas, vol. 17, no. 5, p. 056301, 2010.

[153] D. Hicks, B. Spears, D. Braun, R. Olson, C. Sorce, P. Celliers, G. Collins, and O. Landen, "Streaked radiography measurements of convergent ablator performance," Review of Scientific Instruments, vol. 81, no. 10, p. 10E304, 2010.

[154] D. Hoarty, P. Allan, S. James, C. Brown, L. Hobbs, M. Hill, J. Harris, J. Morton, M. Brookes, R. Shepherd, et al., "Observations of the effect of ionizationpotential depression in hot dense plasma," Physical review letters, vol. 110, no. 26, p. 265003, 2013.

[155] Y. P. Opachich, D. H. Kalantar, A. G. MacPhee, J. P. Holder, J. R. Kimbrough, P. M. Bell, D. K. Bradley, B. Hatch, G. Brienza-Larsen, C. Brown, C. G. Brown, D. Browning, M. Charest, E. L. Dewald, M. Griffin, B. Guidry, M. J. Haugh, D. G. Hicks, D. Homoelle, J. J. Lee, A. J. Mackinnon, A. Mead, N. Palmer, B. H. Perfect, J. S. Ross, C. Silbernagel, and O. Landen, "High performance 
imaging streak camera for the national ignition facility," Review of Scientific Instruments, vol. 83, no. 12, p. 125105, 2012.

[156] Y. Opachich, N. Palmer, D. Homoelle, B. Hatch, P. Bell, D. Bradley, D. Kalantar, D. Browning, J. Zuegel, and O. Landen, "X-ray streak camera cathode development and timing accuracy of the $4 \omega$ ultraviolet fiducial system at the national ignition facility," Review of Scientific Instruments, vol. 83, no. 10, p. 10E123, 2012.

[157] F. Pérez, G. E. Kemp, S. P. Regan, M. A. Barrios, J. Pino, H. Scott, S. Ayers, H. Chen, J. Emig, J. D. Colvin, M. Bedzyk, M. J. Shoup, A. Agliata, B. Yaakobi, F. J. Marshall, R. A. Hamilton, J. Jaquez, M. Farrell, A. Nikroo, and K. B. Fournier, "The NIF x-ray spectrometer calibration campaign at Omega," Review of Scientific Instruments, vol. 85, no. 11, p. 11D613, 2014. 